\title{
Machine learning and the physical sciences
}

\author{
Giuseppe Carleo \\ Center for Computational Quantum Physics, \\ Flatiron Institute, \\ 162 5th Avenue, \\ New York, NY 10010, \\ USA \\ Ignacio Cirac \\ Max-Planck-Institut fur Quantenoptik, \\ Hans-Kopfermann-Straße 1, \\ D-85748 Garching, \\ Germany \\ Kyle Cranmer \\ Center for Cosmology and Particle Physics, \\ Center of Data Science, \\ New York University, 726 Broadway, \\ New York, NY 10003, \\ USA \\ Laurent Daudet \\ LightOn, 2 rue de la Bourse, F-75002 Paris, \\ France \\ Maria Schuld \\ University of KwaZulu-Natal, \\ Durban 4000, South Africa \\ National Institute for Theoretical Physics, \\ KwaZulu-Natal, Durban 4000, South Africa, \\ and Xanadu Quantum Computing, \\ 777 Bay Street, M5B 2 H7 Toronto, \\ Canada \\ Naftali Tishby \\ The Hebrew University of Jerusalem, \\ Edmond Safra Campus, \\ Jerusalem 91904, \\ Israel \\ Leslie Vogt-Maranto \\ Department of Chemistry, \\ New York University, \\ New York, NY 10003, \\ USA

\section{Lenka Zdeborová} \\ Institut de physique théorique, \\ Université Paris Saclay, CNRS, CEA, \\ F-91191 Gif-sur-Yvette, \\ France
}

Machine learning encompasses a broad range of algorithms and modeling tools used for a vast array of data processing tasks, which has entered most scientific disciplines in recent years. We review in a selective way the recent research on the interface between machine learning and physical sciences. This includes conceptual developments in machine learning (ML) motivated by physical insights, applications of machine learning techniques to several domains in physics, and cross-fertilization between the two fields. After giving basic notion of machine learning methods and principles, we describe examples of how statistical physics is used to understand methods in ML. We then move 
to describe applications of ML methods in particle physics and cosmology, quantum many body physics, quantum computing, and chemical and material physics. We also highlight research and development into novel computing architectures aimed at accelerating ML. In each of the sections we describe recent successes as well as domain-specific methodology and challenges.

\section{CONTENTS}

I. Introduction 3

A. Concepts in machine learning

1. Supervised learning and neural networks 4

2. Unsupervised learning and generative modelling 6

3. Reinforcement learning

II. Statistical Physics

A. Historical note

B. Theoretical puzzles in deep learning

C. Statistical physics of unsupervised learning

1. Contributions to understanding basic unsupervised methods

2. Restricted Boltzmann machines

3. Modern unsupervised and generative modelling

D. Statistical physics of supervised learning

1. Perceptron and GLMs

2. Physics results on multi-layer neural networks

3. Information Bottleneck

E. Applications of ML in Statistical Physics

F. Outlook and Challenges 12

III. Particle Physics and Cosmology

A. The role of the simulation 13

B. Classification and regression in particle physics 14

1. Jet Physics 15

2. Neutrino physics 15

3. Robustness to systematic uncertainties 16

4. Triggering 16

5. Theoretical particle physics 17

C. Classification and regression in cosmology

1. Photometric Redshift 17

2. Gravitational lens finding and parameter estimation 18

3. Other examples 18

D. Inverse Problems and Likelihood-free inference

1. Likelihood-free Inference 20

2. Examples in particle physics 20

3. Examples in Cosmology 21

E. Generative Models 22

F. Outlook and Challenges 22

IV. Many-Body Quantum Matter 22

A. Neural-Network quantum states

1. Representation theory 24

2. Learning from data 24

3. Variational Learning 25

B. Speed up many-body simulations

C. Classifying many-body quantum phases

1. Synthetic data 26

2. Experimental data 27

D. Tensor networks for machine learning 27

E. Outlook and Challenges

V. Quantum computing 29

A. Quantum state tomography 29

B. Controlling and preparing qubits

C. Error correction 31

VI. Chemistry and Materials 31

A. Energies and forces based on atomic environments

B. Potential and free energy surfaces

C. Materials properties 33 
D. Electron densities for density functional theory

E. Data set generation

F. Outlook and Challenges

VII. AI acceleration with classical and quantum hardware

A. Beyond von Neumann architectures

B. Neural networks running on light

C. Revealing features in data

D. Quantum-enhanced machine learning

E. Outlook and Challenges

VIII. Conclusions and Outlook

Acknowledgements

References

\section{INTRODUCTION}

The past decade has seen a prodigious rise of machinelearning (ML) based techniques, impacting many areas in industry including autonomous driving, health-care, finance, manufacturing, energy harvesting, and more. ML is largely perceived as one of the main disruptive technologies of our ages, as much as computers have been in the 1980's and 1990's. The general goal of ML is to recognize patterns in data, which inform the way unseen problems are treated. For example, in a highly complex system such as a self-driving car, vast amounts of data coming from sensors have to be turned into decisions of how to control the car by a computer that has "learned" to recognize the pattern of "danger".

The success of ML in recent times has been marked at first by significant improvements on some existing technologies, for example in the field of image recognition. To a large extent, these advances constituted the first demonstrations of the impact that ML methods can have in specialized tasks. More recently, applications traditionally inaccessible to automated software have been successfully enabled, in particular by deep learning technology. The demonstration of reinforcement learning techniques in game playing, for example, has had a deep impact in the perception that the whole field was moving a step closer to what expected from a general artificial intelligence.

In parallel to the rise of ML techniques in industrial applications, scientists have increasingly become interested in the potential of ML for fundamental research, and physics is no exception. To some extent, this is not too surprising, since both ML and physics share some of their methods as well as goals. The two disciplines are both concerned about the process of gathering and analyzing data to design models that can predict the behaviour of complex systems. However, the fields prominently differ

\footnotetext{
* Corresponding author: gcarleo@flatironinstitute.org

† Corresponding author: lenka.zdeborova@cea.fr
}

in the way their fundamental goals are realized. On the one hand, physicists want to understand the mechanisms of Nature, and are proud of using their own knowledge, intelligence and intuition to inform their models. On the other hand, machine learning mostly does the opposite: models are agnostic and the machine provides the 'intelligence' by extracting it from data. Although often powerful, the resulting models are notoriously known to be as opaque to our understanding as the data patterns themselves. Machine learning tools in physics are therefore welcomed enthusiastically by some, while being eyed with suspicions by others. What is difficult to deny is that they produce surprisingly good results in some cases.

In this review, we attempt at providing a coherent selected account of the diverse intersections of ML with physics. Specifically, we look at an ample spectrum of fields (ranging from statistical and quantum physics to high energy and cosmology) where ML recently made a prominent appearance, and discuss potential applications and challenges of 'intelligent' data mining techniques in the different contexts. We start this review with the field of statistical physics in Section II] where the interaction with machine learning has a long history, drawing on methods in physics to provide better understanding of problems in machine learning. We then turn the wheel in the other direction of using machine learning for physics. Section III treats progress in the fields of high-energy physics and cosmology, Section IV reviews how ML ideas are helping to understand the mysteries of many-body quantum systems, Section V briefly explore the promises of machine learning within quantum computations, and in Section VI] we highlight some of the amazing advances in computational chemistry and materials design due to ML applications. In Section VII we discuss some advances in instrumentation leading potentially to hardware adapted to perform machine learning tasks. We conclude with an outlook in Section VIII. 


\section{A. Concepts in machine learning}

For the purpose of this review we will briefly explain some fundamental terms and concepts used in machine learning. For further reading, we recommend a few resources, some of which have been targeted especially for a physics audience. For a historical overview of the development of the field we recommend Refs. (LeCun et al., 2015, Schmidhuber, 2014). An excellent recent introduction to machine learning for physicists is Ref. (Mehta et al. 2018), which includes notebooks with practical demonstrations. A very useful online resource is Florian Marquardt's course "Machine learning for physicists' 1 . Useful textbooks written by machine learning researchers are Christopher Bishop's standard textbook (Bishop, 2006), as well as (Goodfellow et al. 2016) which focuses on the theory and foundations of deep learning and covers many aspects of current-day research. A variety of online tutorials and lectures is useful to get a basic overview and get started on the topic.

To learn about the theoretical progress made in statistical physics of neural networks in the 1980s-1990s we recommend the rather accessible book Statistical Mechanics of Learning (Engel and Van den Broeck, 2001). For learning details of the replica method and its use in computer science, information theory and machine learning we would recommend the book of Nishimori (Nishimori, 2001). For the more recent statistical physics methodology the textbook of Mézard and Montanari is an excellent reference (Mézard and Montanari, 2009).

To get a basic idea of the type of problems that machine learning is able to tackle it is useful to defined three large classes of learning problems: Supervised learning, unsupervised learning and reinforcement learning. This will also allow us to state the basic terminology, building basic equipment to expose some of the basic tools of machine learning.

\section{Supervised learning and neural networks}

In supervised learning we are given a set of $n$ samples of data, let us denote one such sample $X_{\mu} \in \mathbb{R}^{p}$, with $\mu=1, \ldots, n$. To have something concrete in mind each $X_{\mu}$ could be for instance a black-and-white photograph of an animal, and $p$ the number of pixels. For each sample $X_{\mu}$ we are further given a label $y_{\mu} \in \mathbb{R}^{d}$, most commonly $d=1$. The label could encode for instance the species of the animal on the photograph. The goal of supervised learning is to find a function $f$ so that when a new sample $X_{\text {new }}$ is presented without its label, then the output of the function $f\left(X_{\text {new }}\right)$ approximates well the label. The data

1 See https://machine-learning-for-physicists.org/ set $\left\{X_{\mu}, y_{\mu}\right\}_{\mu=1, \ldots, n}$ is called the training set. In order to test the resulting function $f$ one usually splits the available data samples into the training set used to learn the function and a test set to evaluate the performance.

Let us now describe the training procedure most commonly used to find a suitable function $f$. Most commonly the function is expressed in terms of a set of parameters, called weights $w \in \mathbb{R}^{k}$, leading to $f_{w}$. One then constructs a so-called loss function $\mathcal{L}\left[f_{w}\left(X_{\mu}\right), y_{\mu}\right]$ for each sample $\mu$, with the idea of this loss being small when $f_{w}\left(X_{\mu}\right)$ and $y_{\mu}$ are close, and vice versa. The average of the loss over the training set is then called the empirical $\operatorname{risk} \mathcal{R}\left(f_{w}\right)=\sum_{\mu=1}^{n} \mathcal{L}\left[f_{w}\left(X_{\mu}\right), y_{\mu}\right] / n$.

During the training procedure the weights $w$ are being adjusted in order to minimize the empirical risk. The training error measures how well is such a minimization achieved. A notion of error that is the most important is the generalization error, related to the performance on predicting labels $y_{\text {new }}$ for data samples $X_{\text {new }}$ that were not seen in the training set. In applications, it is common practice to build the test set by randomly picking a fraction of the available data, and perform the training using the remaining fraction as a training set. We note that in a part of the literature the generalization error is the difference between the performance of the test set and the one on the training set.

The algorithms most commonly used to minimize the empirical risk function over the weights are based on gradient descent with respect to the weights $w$. This means that the weights are iteratively adjusted in the direction of the gradient of the empirical risk

$$
w^{t+1}=w^{t}-\gamma \nabla_{w} \mathcal{R}\left(f_{w}\right)
$$

The rate $\gamma$ at which this is performed is called the learning rate. A very commonly used and successful variant of the gradient descent is the stochastic gradient descent (SGD) where the full empirical risk function $\mathcal{R}$ is replaced by the contribution of just a few of the samples. This subset of samples is called mini-batch and can be as small as a single sample. In physics terms, the SGD algorithm is often compared to the Langevin dynamics at finite temperature. Langevin dynamics at zero temperature is the gradient descent. Positive temperature introduces a thermal noise that is in certain ways similar to the noise arising in SGD, but different in others. There are many variants of the SGD algorithm used in practice. The initialization of the weights can change performance in practice, as can the choice of the learning rate and a variety of so-called regularization terms, such as weight decay that is penalizing weights that tend to converge to large absolute values. The choice of the right version of the algorithm is important, there are many heuristic rules of thumb, and certainly more theoretical insight into the question would be desirable.

One typical example of a task in supervised learning is classification, that is when the labels $y_{\mu}$ take values 
in a discrete set and the so-called accuracy is then measured as the fraction of times the learned function classifies the data point correctly. Another example is regression where the goal is to learn a real-valued function, and the accuracy is typically measured in terms of the mean-squared error between the true labels and their learned estimates. Other examples would be sequenceto-sequence learning where both the input and the label are vectors of dimension larger than one.

There are many methods of supervised learning and many variants of each. One of the most basic supervised learning method is the widely known and used linear regression, where the function $f_{w}(X)$ is parameterized in the form $f_{w}\left(X_{\mu}\right)=X_{\mu} w$, with $w \in \mathbb{R}^{p}$. When the data live in high dimensional space and the number of samples is not much larger than the dimension, it is indispensable to use regularized form of linear regression called ridge regression or Tikhonov regularization. The ridge regression is formally equivalent to assuming that the weights $w$ have a Gaussian prior. A generalized form of linear regression, with parameterization $f_{w}\left(X_{\mu}\right)=g\left(X_{\mu} w\right)$, where $g$ is some output channel function, is also often used and its properties are described in section II.D.1. Another popular way of regularization is based on separating the example $n$ a classification task so that they the separate categories are divided by a clear gap that is as wide as possible. This idea stands behind the definition of so-called support vector machine method.

A rather powerful non-parametric generalization of the ridge regression is kernel ridge regression. Kernel ridge regression is closely related to Gaussian process regression. The support vector machine method is often combined with a kernel method, and as such is still the stateof-the-art method in many applications, especially when the number of available samples is not very large.

Another classical supervised learning method is based on so-called decision trees. The decision tree is used to go from observations about a data sample (represented in the branches) to conclusions about the item's target value (represented in the leaves). The best known application of decision trees in physical science is in data analysis of particle accelerators, as discussed in Sec. III.B.

The supervised learning method that stands behind the machine learning revolution of the past decade are multi-layer feed-forward neural networks (FFNN) also sometimes called multi-layer perceptrons. This is also a very relevant method for the purpose of this review and we shall describe it briefly here. In L-layer fully connected neural networks the function $f_{w}\left(X_{\mu}\right)$ is parameterized as follows

$$
f_{w}\left(X_{\mu}\right)=g^{(L)}\left(W^{(L)} \ldots g^{(2)}\left(W^{(2)} g^{(1)}\left(W^{(1)} X_{\mu}\right)\right)\right),
$$

where $w=\left\{W^{(1)}, \ldots, W^{(L)}\right\}_{i=1, \ldots, L}$, and $W^{(i)} \in$ $\mathbb{R}^{r_{i} \times r_{i-1}}$ with $r_{0}=p$ and $r_{L}=d$, are the matrices of weights, and $r_{i}$ for $1 \leq i \leq L-1$ is called the width of the $i$ - th hidden layer. The functions $g^{(i)}, 1 \leq i \leq L$, are the so-called activation functions, and act componentwise on vectors. We note that the input in the activation functions are often slightly more generic affine transforms of the output of the previous layer that simply matrix multiplications, including e.g. biases. The number of layers $L$ is called the network's depth. Neural networks with depth larger than some small integer are called deep neural networks. Subsequently machine learning based on deep neural networks is called deep learning.

The theory of neural networks tells us that without hidden layers ( $L=1$, corresponding to the generalized linear regression) the set of functions that can be approximated this way is very limited (Minsky and Papert, 1969). On the other hand already with one hidden layer, $L=2$, that is wide enough, i.e. $r_{1}$ large enough, and where the function $g^{(1)}$ is non-linear, a very general class of functions can be well approximated in principle $(\mathrm{Cy}-$ benko, 1989). These theories, however, do not tell us what is the optimal set of parameters (the activation functions, the widths of the layers and the depth) in order for the learning of $W^{(1)}, \ldots, W^{(L)}$ to be tractable efficiently. We know from empirical success of the past decade that many tasks of interest are tractable with deep neural network using the gradient descent or the SGD algorithms. In deep neural networks the derivatives with respect to the weights are computed using the chain rule leading to the celebrated back-propagation algorithm that takes care of efficiently scheduling the operations required to compute all the gradients (Goodfellow et al. 2016).

A very important and powerful variant of (deep) feedforward neural networks are the so-called convolutional neural networks (Goodfellow et al., 2016) where the input into each of the hidden units is obtained via a filter applied to a small part of the input space. The filter is then shifted to different positions corresponding to different hidden units. Convolutional neural networks implement invariance to translation and are in particular suitable for analysis of images. Compared to the fully connected neural networks each layer of convolutional neural network has much smaller number of parameters, which is in practice advantageous for the learning algorithms. There are many types and variances of convolutional neural networks, among them we will mention the Residual neural networks (ResNets) use shortcuts to jump over some layers.

Next to feed-forward neural networks there are the socalled recurrent neural networks (RNN) in which the outputs of units feeds back at the input in the next time step. In RNNs the result is thus given by the set of weights, but also by the whole temporal sequence of states. Due to their intrinsically dynamical nature, RNN are particularly suitable for learning for temporal data sets, such as speech, language, and time series. Again there are many types and variants on RNNs, but the ones that caused the most excitement in the past decade are arguably the 
long short-term memory (LSTM) networks (Hochreiter and Schmidhuber, 1997). LSTMs and their deep variants are the state-of-the-art in tasks such as speech processing, music compositions, and natural language processing.

\section{Unsupervised learning and generative modelling}

Unsupervised learning is a class of learning problems where input data are obtained as in supervised learning, but no labels are available. The goal of learning here is to recover some underlying -and possibly non-trivialstructure in the dataset. A typical example of unsupervised learning is data clustering where data points are assigned into groups in such a way that every group has some common properties.

In unsupervised learning, one often seeks a probability distribution that generates samples that are statistically similar to the observed data samples, this is often referred to as generative modelling. In some cases this probability distribution is written in an explicit form and explicitly or implicitly parameterized. Generative models internally contain latent variables as the source of randomness. When the number of latent variables is much smaller than the dimensionality of the data we speak of dimensionality reduction. One path towards unsupervised learning is to search values of the latent variables that maximize the likelihood of the observed data.

In a range of applications the likelihood associated to the observed data is not known or computing it is itself intractable. In such cases, some of the generative models discussed below offer on alternative likelihoodfree path. In Section III.D we will also discuss the socalled $A B C$ method that is a type of likelihood-free inference and turns out to be very useful in many contexts arising in physical sciences.

Basic methods of unsupervised learning include principal component analysis and its variants. We will cover some theoretical insights into these method that were obtained using physics in section II.C.1. A physically very appealing methods for unsupervised learning are the socalled Boltzmann machines (BM). A BM is basically an inverse Ising model where the data samples are seen as samples from a Boltzmann distribution of a pair-wise interacting Ising model. The goal is to learn the values of the interactions and magnetic fields so that the likelihood (probability in the Boltzmann measure) of the observed data is large. A restricted Boltzmann machine (RBM) is a particular case of BM where two kinds of variables visible units, that see the input data, and hidden units, interact through effective couplings. The interactions are in this case only between visible and hidden units and are again adjusted in order for the likelihood of the observed data to be large. Given the appealing interpretation in terms of physical models, applications of BMs and RBMs are widespread in several physics domains, as discussed e.g. in section IV.A.

A very neat idea to perform unsupervised learning yet being able to use all the methods and algorithms developed for supervised learning are auto-encoders. An autoencoder is a feed-forward neural network that has the input data on the input, but also on the output. It aims to reproduce the data while typically going trough a bottleneck, in the sense that some of the intermediate layers have very small width compared to the dimensionality of the data. The idea is then that autoencoder is aiming to find a succinct representation of the data that still keeps the salient features of each of the samples. Variational autoencoders (VAE) (Kingma and Welling, 2013: Rezende et al., 2014) combine variational inference and autoencoders to provide a deep generative model for the data, which can be trained in an unsupervised fashion.

A further approach to unsupervised learning worth mentioning here, are adversarial generative networks (GANs) (Goodfellow et al., 2014). GANs have attracted substantial attentions in the past years, and constitute another fruitful way to take advantage of the progresses made for supervised learning to do unsupervised one. GANs typical use two feed-forward neural networks, one called the generator and another called the discriminator. The generator network is used to generate outputs from random inputs, and is designed so that the outputs look like the observed samples. The discriminator network is used to discriminate between true data samples and samples generated by the generator network. The discriminator is aiming at best possible accuracy in this classification task, whereas the generator network is adjusted to make the accuracy of the discriminator the smallest possible. GANs currently are the state-of-the art system for many applications in image processing.

Other interesting methods to model distributions include normalizing flows and autoregressive models with the advantage of having tractable likelihood so that they can be trained via maximum likelihood (Larochelle and Murray, 2011, Papamakarios et al., 2017, Uria et al., 2016).

Hybrids between supervised learning and unsupervised learning that are important in application include semisupervised learning where only some labels are available, or active learning where labels can be acquired for a selected set of data points at a certain cost.

\section{Reinforcement learning}

Reinforcement learning (Sutton and Barto, 2018) is an area of machine learning where an (artificial) agent takes actions in an environment with the goal of maximizing a reward. The action changes the state of the environment in some way and the agent typically observes some information about the state of the environment and the corresponding reward. Based on those observations the 
agent decides on the next action, refining the strategies of which action to choose in order to maximize the resulting reward. This type of learning is designed for cases where the only way to learn about the properties of the environment is to interact with it. A key concept in reinforcement learning it the trade-off between exploitation of good strategies found so far, and exploration in order to find yet better strategies. We should also note that reinforcement learning is intimately related to the field of theory of control, especially optimal control theory.

One of the main types of reinforcement learning applied in many works is the so-called Q-learning. Qlearning is based on a value matrix $Q$ that assigns quality of a given action when the environment is in a given state. This value function $Q$ is then iteratively refined. In recent advanced applications of Q-learning the set of states and action is so large that it is impossible to even store the whole matrix $Q$. In those cases deep feed-forward neural networks are used to represent the function in a succinct manner. This gives rise to deep Q-learning.

Most well-known recent examples of the success of reinforcement learning is the computer program AlphaGo and AlphaGo Zero that for a first time in history reached super-human performance in the traditional board game of Go. Another well known use of reinforcement learning is locomotion of robots.

\section{STATISTICAL PHYSICS}

\section{A. Historical note}

While machine learning as a wide-spread tool for physics research is a relatively new phenomenon, crossfertilization between the two disciplines dates back much further. Especially statistical physicists made important contributions to our theoretical understanding of learning (as the term "statistical" unmistakably suggests).

The connection between statistical mechanics and learning theory started when statistical learning from examples took over the logic and rule based AI, in the mid 1980s. Two seminal papers marked this transformation, Valiant's theory of the learnable (Valiant, 1984), which opened the way for rigorous statistical learning in AI, and Hopfield's neural network model of associative memory (Hopfield, 1982), which sparked the rich application of concepts from spin glass theory to neural networks models. This was marked by the memory capacity calculation of the Hopfield model by Amit, Gutfreund, and Sompolinsky (Amit et al. 1985) and following works. A much tighter application to learning models was made by the seminal work of Elizabeth Gardner who applied the replica trick (Gardner, 1987, 1988) to calculate volumes in the weights space for simple feed-forward neural networks, for both supervised and unsupervised learning models.
Gardner's method enabled to explicitly calculate learning curves, i.e. the typical training and generalization errors as a function of the number of training examples, for very specific one and two-layer neural networks (Györgyi and Tishby, 1990; Seung et al., 1992a, Sompolinsky et al., 1990). These analytic statistical physics calculations demonstrated that the learning dynamics can exhibit much richer behavior than predicted by the worsecase distribution free PAC bounds (PAC stands for provably approximately correct) (Valiant, 1984). In particular, learning can exhibit phase transitions from poor to good generalization (Györgyi 1990). This rich learning dynamics and curves can appear in many machine learning problems, as was shown in various models, see e.g. more recent review (Zdeborová and Krzakala, 2016). The statistical physics of learning reached its peak in the early 1990s, but had rather minor influence on machinelearning practitioners and theorists, who were focused on general input-distribution-independent generalization bounds, characterized by e.g. the Vapnik-Chervonenkis dimension or the Rademacher complexity of hypothesis classes.

\section{B. Theoretical puzzles in deep learning}

Machine learning in the new millennium was marked by much larger scale learning problems, in input/pattern sizes which moved from hundreds to millions in dimensionality, in training data sizes, and in number of adjustable parameters. This was dramatically demonstrated by the return of large scale feed-forward neural network models, with many more hidden layers, known as deep neural networks. These deep neural networks were essentially the same feed-forward convolution neural networks proposed already in the 80s. But somehow with the much larger scale inputs and big and clean training data (and a few more tricks and hacks), these networks started to beat the state-of-the-art in many different pattern recognition and other machine learning competitions, from roughly 2010 and on. The amazing performance of deep learning, trained with the same old stochastic gradient descent (SGD) error-backpropagation algorithm, took everyone by surprise.

One of the puzzles is that the existing learning theory (based on the worst-case PAC-like generalization bounds) is unable to explain this phenomenal success. The existing theory does not predict why deep networks, where the number/dimension of adjustable parameters/weights is way higher than the number of training samples, have good generalization properties. This lack of theory was coined in now a classical article (Zhang et al., 2016), where the authors show numerically that state-of-the-art neural networks used for classification are able to classify perfectly randomly generated labels. In such a case existing learning theory does not provide any useful bound on 
the generalization error. Yet in practice we observe good generalization of the same deep neural networks when trained on the true labels.

Continuing with the open question, we do not have good understanding of which learning problems are computationally tractable. This is particularly important since from the point of view of computational complexity theory, most of the learning problems we encounter are NP-hard in the worst case. Another open question that is central to current deep learning concern the choice of hyper-parameters and architectures that is so far guided by a lot of trial-and-error combined by impressive experience of the researchers. At the same time as applications of ML are spreading into many domains, the field calls for more systematic and theory-based approaches. In current deep-learning, basic questions such as what is the minimal number of samples we need in order to be able to learn a given task with a good precision is entirely open.

At the same time the current literature on deep learning is flourishing with interesting numerical observations and experiments that call for explanation. For a physics audience the situation could perhaps be compared to the state-of-the-art in fundamental small-scale physics just before quantum mechanics was developed. The field was full of unexplained experiments that were evading existing theoretical understanding. This clearly is the perfect time for some of physics ideas to study neural networks to resurrect and revisit some of the current questions and directions in machine learning.

Given the long history of works done on neural networks in statistical physics, we will not aim at a complete review of this direction of research. We will focus in a selective way on recent contributions originating in physics that, in our opinion, are having important impact in current theory of learning and machine learning. For the purpose of this review we are also putting aside a large volume of work done in statistical physics on recurrent neural networks with biological applications in mind.

\section{Statistical physics of unsupervised learning}

1. Contributions to understanding basic unsupervised methods

One of the most basic tools of unsupervised learning across the sciences are methods based on low-rank decomposition of the observed data matrix. Data clustering, principal component analysis (PCA), independent component analysis (ICA), matrix completion, and other methods are examples in this class.

In mathematical language the low-rank matrix decomposition problem is stated as follows: We observe $n$ samples of $p$-dimensional data $x_{i} \in \mathbb{R}^{p}, i=1, \ldots, n$. Denoting $X$ the $n \times p$ matrix of data, the idea underlying low-rank decomposition methods assumes that $X$ (or some component-wise function of $X$ ) can be written as a noisy version of a rank $r$ matrix where $r \ll p ; r \ll n$, i.e. the rank is much lower that the dimensionality and the number of samples, therefore the name low-rank. A particularly challenging, yet relevant and interesting regime, is when the dimensionality $p$ is comparable to the number of samples $n$, and when the level of noise is large in such a way that perfect estimation of the signal is not possible. It turns out that the low-rank matrix estimation in the high-dimensional noisy regime can be modelled as a statistical physics model of a spin glass with $r$-dimensional vector variables and a special planted configuration to be found.

Concretely, this model can be defined in the teacherstudent scenario in which the teacher generates $r$ dimensional latent variables $u_{i}^{*} \in \mathbb{R}^{r}, i=1, \ldots, n$, taken from a given probability distribution $P_{u}\left(u_{i}^{*}\right)$, and $r$-dimensional latent variables $v_{j}^{*} \in \mathbb{R}^{r}, j=1, \ldots, p$, taken from a given probability distribution $P_{v}\left(v_{i}^{*}\right)$. Then the teacher generates components of the data matrix $X$ from some given conditional probability distribution $P_{\text {out }}\left(X_{i j} \mid u_{i}^{*} \cdot v_{j}^{*}\right)$. The goal of the student is then to recover the latent variables $u^{*}$ and $v^{*}$ as precisely as possible from the knowledge of $X$, and the distributions $P_{\text {out }}$, $P_{u}, P_{v}$.

Spin glass theory can be used to obtain rather complete understanding of this teacher-student model for low-rank matrix estimation in the limit $p, n \rightarrow \infty$, $n / p=\alpha=\Omega(1), r=\Omega(1)$. One can compute with the replica method what is the information-theoretically best error in estimation of $u^{*}$, and $v^{*}$ the student can possibly achieve, as done decades ago for some special choices of $r$, $P_{\text {out }}, P_{u}$ and $P_{v}$ in (Barkai and Sompolinsky, 1994, Biehl and Mietzner, 1993: Watkin and Nadal, 1994). The importance of these early works in physics is acknowledged in some of the landmark papers on the subject in statistics, see e.g. (Johnstone and Lu, 2009). However, the lack of mathematical rigor and limited understanding of algorithmic tractability caused the impact of these works in machine learning and statistics to remain limited.

A resurrection of interest in statistical physics approach to low-rank matrix decompositions came with the study of the stochastic block model for detection of clusters/communities in sparse networks. The problem of community detection was studied heuristically and algorithmically extensively in statistical physics, for a review see (Fortunato, 2010). However, the exact solution and understanding of algorithmic limitations in the stochastic block model came from the spin glass theory in (Decelle et al. 2011a b). These works computed (nonrigorously) the asymptotically optimal performance and delimited sharply regions of parameters where this performance is reached by the belief propagation (BP) algorithm (Yedidia et al. 2003). Second order phase transitions appearing in the model separate a phase where clustering cannot be performed better than by random 
guessing, from a region where it can be done efficiently with BP. First order phase transitions and one of their spinodal lines then separate regions where clustering is impossible, possible but not doable with the BP algorithm, and easy with the BP algorithm. Refs. (Decelle et al. 2011a b) also conjectured that when the BP algorithm is not able to reach the optimal performance on large instances of the model, then no other polynomial algorithm will. These works attracted a large amount of follow-up work in mathematics, statistics, machine learning and computer science communities.

The statistical physics understanding of the stochastic block model and the conjecture about belief propagation algorithm being optimal among all polynomial ones inspired the discovery of a new class of spectral algorithms for sparse data (i.e. when the matrix $X$ is sparse) (Krzakala et al. 2013b). Spectral algorithms are basic tools in data analysis ( $\mathrm{Ng}$ et al., 2002; Von Luxburg, 2007), based on the singular value decomposition of the matrix $X$ or functions of $X$. Yet for sparse matrices $X$, the spectrum is known to have leading singular values with localized singular vectors unrelated to the latent underlying structure. A more robust spectral method is obtained by linearizing the belief propagation, thus obtaining a socalled non-backtracking matrix (Krzakala et al. 2013b). A variant on this spectral method based on algorithmic interpretation of the Hessian of the Bethe free energy also originated in physics (Saade et al. 2014).

This line of statistical-physics inspired research is merging into the mainstream in statistics and machine learning. This is largely thanks to recent progress in: (a) our understanding of algorithmic limitations, due to the analysis of approximate message passing (AMP) algorithms (Bolthausen, 2014, Deshpande and Montanari, 2014; Javanmard and Montanari, 2013; Matsushita and Tanaka, 2013; Rangan and Fletcher, 2012) for lowrank matrix estimation that is a generalization of the Thouless-Anderson-Palmer equations (Thouless et al. 1977) well known in the physics literature on spin glasses. And (b) progress in proving many of the corresponding results in a mathematically rigorous way. Some of the influential papers in this direction (related to low-rank matrix estimation) are (Barbier et al. 2016 , Coja-Oghlan et al., 2018; Deshpande and Montanari, 2014; Lelarge and Miolane (2016) for the proof of the replica formula for the information-theoretically optimal performance.

\section{Restricted Boltzmann machines}

Boltzmann machines and in particular restricted Boltzmann machines are another method for unsupervised learning often used in machine learning. As apparent from the very name of the method, it had strong relation with statistical physics. Indeed the Boltzmann machine is often called the inverse Ising model in the physics lit- erature and used extensively a range of area, for a recent review on the physics of Boltzmann machines see (Nguyen et al., 2017).

Concerning restricted Boltzmann machines, there are number of studies in physics clarifying how these machines work and what structures can they learn. Model of random restricted Boltzmann machine, where the weights are imposed to be random and sparse, and not learned, is studied in (Cocco et al. 2018 ; Tubiana and Monasson, 2017). Rather remarkably for a range of potentials on the hidden unit this work unveiled that even the single layer RBM is able to represent compositional structure. Insights from this work were more recently used to model protein families from their sequence information (Tubiana et al. 2018).

Analytical study of the learning process in RBM, that is most commonly done using the contrastive divergence algorithm based on Gibbs sampling (Hinton, 2002), is very challenging. First steps were studied in (Decelle et al. 2017) at the beginning of the learning process where the dynamics can be linearized. Another interesting direction coming from statistical physics is to replace the Gibbs sampling in the contrastive divergence training algorithm by the Thouless-Anderson-Palmer equations (Thouless et al., 1977). This has been done in (Gabrié et al. 2015 (Tramel et al. 2018) where such training was shown to be competitive and applications of the approach were discussed. RBM with random weights and their relation to the Hopfield model was clarified in (Barra et al. 2018; Mézard, 2017).

\section{Modern unsupervised and generative modelling}

The dawn of deep learning brought an exciting innovations into unsupervised and generative-models learning. A physics friendly overview of some classical and more recent concepts is e.g. (Wang, 2018).

Auto-encoders with linear activation functions are closely related to PCA. Variational autoencoders (VAE) Kingma and Welling, 2013, Rezende et al., 2014) are variants much closer to a physicist mind set where the autoencoder is represented via a graphical model, and in trained using a prior on the latent variables and variational inference. VAE with a single hidden layer is closely related to other widely used techniques in signal processing such as dictionary learning and sparse coding. Dictionary learning problem has been studied with statistical physics techniques in (Kabashima et al., 2016, Krzakala et al., 2013a; Sakata and Kabashima, 2013).

Generative adversarial networks (GANs) - a powerful set of ideas emerged with the work of (Goodfellow et al. 2014) aiming to generate samples (e.g. images of hotel bedrooms) that are of the same type as those in the training set. Physics-inspired studies of GANs are starting to appear, e.g. the work on a solvable model of GANs by 
(Wang et al. 2018) is a intriguing generalization of the earlier statistical physics works on online learning in perceptrons.

We also want to point the readers attention to autoregressive generative models (Larochelle and Murray, 2011, Papamakarios et al., 2017, Uria et al.|, 2016). The main interest in autoregressive models stems from the fact that they are a family of explicit probabilistic models, for which direct and unbiased sampling is possible. Applications of these models have been realized for both statistical (Wu et al. 2018$)$ and quantum physics problems (Sharir et al. |2019).

\section{Statistical physics of supervised learning}

\section{Perceptron and GLMs}

The arguably most basic method of supervised learning is linear regression where one aims to find a vector of coefficients $w$ so that its scalar product with the data point $X_{i} w$ corresponds to the observed predicate $y$. This is most often solved by the least squares method where $\|y-X w\|_{2}^{2}$ is minimized over $w$. In the Bayesian language, the least squares method corresponds to assuming Gaussian additive noise $\xi$ so that $y_{i}=X_{i} w+\xi_{i}$. In high dimensional setting it is almost always indispensable to use regularization of the weights. The most common ridge regularization corresponds in the Bayesian interpretation to Gaussian prior on the weights. This probabilistic thinking can be generalized by assuming a general prior $P_{W}(\cdot)$ and a generic noise represented by a conditional probability distribution $P_{\text {out }}\left(y_{i} \mid X_{i} w\right)$. The resulting model is called generalized linear regression or generalized linear model (GLM). Many other problems of interest in data analysis and learning can be represented as GLM. For instance sparse regression simply requires that $P_{W}$ has large weight on zero, for the perceptron with threshold $\kappa$ the output has a special form $P_{\text {out }}(y \mid z)=\mathbb{I}(z>\kappa) \delta(y-1)+\mathbb{I}(z \leq \kappa) \delta(y+1)$. In the language of neural networks, the GLM represents a single layer (no hidden variables) fully connected feed-forward network.

For generic noise/activation channel $P_{\text {out }}$ traditional theories in statistics are not readily applicable to the regime of very limited data where both the dimension $p$ and the number of samples $n$ grow large, while their ratio $n / p=\alpha$ remains fixed. Basic questions such as: how does the best achievable generalization error depend on the number of samples, remain open. Yet this regime and related questions are of great interest and understanding them well in the setting of GLM seems to be a prerequisite to understand more involved, e.g. deep learning, methods.

Statistical physics approach can be used to obtain specific results on the high-dimensional GLM by considering data to be random independent identically distributed (iid) matrix and modelling the labels as being created in the teacher-student setting. The teacher generates a ground-truth vector of weights $w$ so that $w_{j} \sim P_{w}$, $j=1, \ldots, p$. The teacher then uses this vector and data matrix $X$ to produce labels $y$ taken from $P_{\text {out }}\left(y_{i} \mid X_{i} w^{*}\right)$. The students then knows $X, y, P_{w}$ and $P_{\text {out }}$ and is supposed to learn the rule the teacher uses, i.e. ideally to learn the $w^{*}$. Already this setting with random input data provides interesting insights into the algorithmic tractability of the problem as the number of samples changes.

This line of work was pioneered by Elisabeth Gardner (Gardner and Derrida, 1989) and actively studied in physics in the past for special cases of $P_{\text {out }}$ and $P_{W}$, see e.g. (Györgyi and Tishby, 1990; Seung et al., 1992a; Sompolinsky et al., 1990). The replica method can be used to compute the mutual information between $X$ and $y$ in this teacher-student model, which is related to the free energy in physics. One can then deduce the optimal estimation error of the vector $w^{*}$, as well as the optimal generalization error. A remarkable recent progress was made in (Barbier et al., 2019) where it has been proven that the replica method yields the correct results for the GLM with random inputs for generic $P_{\text {out }}$ and $P_{W}$. Combining these results with the analysis of the approximate message passing algorithms (Javanmard and Montanari 2013), one can deduce cases where the AMP algorithm is able to reach the optimal performance and regions where it is not. The AMP algorithm is conjectured to be the best of all polynomial algorithm for this case. The teacher-student model could thus be used by practitioners to understand how far from optimality are general purpose algorithms in cases where only very limited number of samples is available.

\section{Physics results on multi-layer neural networks}

Statistical physics analysis of learning and generalization properties in deep neural networks is a challenging task. Progress had been made in several complementary directions.

One of the influential directions involved studies of linear deep neural networks. While linear neural networks do not have the expressive power to represent generic functions, the learning dynamics of the gradient descent algorithm bears strong resemblance with the learning dynamics on non-linear networks. At the same time the dynamics of learning in deep linear neural networks can be described via a closed form solution (Saxe et al., 2013 ). The learning dynamics of linear neural networks is also able to reproduce a range of facts about generalization and over-fitting as observed numerically in non-linear networks, see e.g. (Advani and Saxe, 2017).

Another special case that has been analyzed in great 
detail is called the committee machine, for a review see e.g. (Engel and Van den Broeck, 2001). Committee machine is a fully-connected neural network learning a teacher-rule on random input data with only the first layer of weights being learned, while the subsequent ones are fixed. The theory is restricted to the limit where the number of hidden neurons $k=O(1)$, while the dimensionality of the input $p$ and the number of samples $n$ are both diverge, with $n / p=\alpha=O(1)$. Both the stochastic gradient descent (aka online) learning (Saad and Solla, 1995a b) and the optimal batch-learning generalization error can be analyzed in closed form in this case (Schwarze, 1993). Recently the replica analysis of the optimal generalization properties has been established rigorously (Aubin et al. 2018). A key feature of the committee machine is that it displays the so-called specialization phase transition. When the number of samples is small, the optimal error is achieved by a weight-configuration that is the same for every hidden unit, effectively implementing simple regression. Only when the number of hidden units exceeds the specialization threshold the different hidden units learn different weights resulting in improvement of the generalization error. Another interesting observation about the committee machine is that the hard phase where good generalization is achievable information-theoretically but not tractably gets larger as the number of hidden units grows. Committee machine was also used to analyzed the consequences of over-parametrization in neural networks in (Goldt et al. $2019 \mathrm{a}$ b).

Another remarkable limit of two-layer neural networks was analysed in a recent series of works (Mei et al. 2018; Rotskoff and Vanden-Eijnden, 2018). In these works the networks are analysed in the limit where the number of hidden units is large, while the dimensionality of the input is kept fixed. In this limit the weights interact only weakly - leading to the term mean field - and their evolution can be tracked via an ordinary differential equation analogous to those studied in glassy systems (Dean, 1996). A related, but different, treatment of the limit when the hidden layers are large is based on linearization of the dynamics around the initial condition leading to relation with Gaussian processes and kernel methods, see e.g. (Jacot et al., 2018, Lee et al., 2018)

\section{Information Bottleneck}

Information bottleneck (Tishby et al. 2000 ) is another concept stemming in statistical physics that has been influential in the quest for understanding the theory behind the success of deep learning. The theory of the information bottleneck for deep learning (Shwartz-Ziv and Tishby, 2017, Tishby and Zaslavsky, 2015) aims to quantify the notion that layers in a neural networks are trading off between keeping enough information about the input so that the output labels can be predicted, while forgetting as much of the unnecessary information as possible in order to keep the learned representation concise.

One of the interesting consequences of this information theoretic analysis is that the traditional capacity, or expressivity dimension of the network, such as the VC dimension, is replaced by the exponent of the mutual information between the input and the compressed hidden layer representation. This implies that every bit of representation compression is equivalent to doubling the training data in its impact on the generalization error.

The analysis of (Shwartz-Ziv and Tishby, 2017) also suggests that such representation compression is achieved by Stochastic Gradient Descent (SGD) through diffusion in the irrelevant dimensions of the problem. According to this, compression is achieved with any units nonlinearity by reducing the SNR of the irrelevant dimensions, layer by layer, through the diffusion of the weights. An intriguing prediction of this insight is that the time to converge to good generalization scales like a negative power-law of the number of layers. The theory also predicts a connection between the hidden layers and the bifurcations, or phase transitions, of the Information Bottleneck representations.

While the mutual information of the internal representations is intrinsically hard to compute directly in large neural networks, none of the above predictions depend on explicit estimation of mutual information values.

A related line of work in statistical physics aims to provide reliable scalable approximations and models where the mutual information is tractable. The mutual information can be computed exactly in linear networks (Saxe et al. 2018). It can be reliably approximated in models of neural networks where after learning the matrices of weights are close enough to rotationally invariant, this is then exploited within the replica theory in order to compute the desired mutual information (Gabrié et al. 2018).

\section{Landscapes and glassiness of deep learning}

Training a deep neural network is usually done via stochastic gradient descent (SGD) in the non-convex landscape of a loss function. Statistical physics has long experience in studies of complex energy landscapes and and their relation to dynamical behaviour. Gradient descent algorithms are closely related to the Langevin dynamics that is often considered in physics. Some physicsinspired works (Choromanska et al. 2015) became popular but were somewhat naive in exploring this analogy.

Interesting insight on the relation between glassy dynamics and learning in deep neural networks is presented in (Baity-Jesi et al. 2018 ). In particular the role of over-parameterization in making the landscape look less glassy is highlighted and contrasted with the under- 
parametrized networks.

Another intriguing line of work that relates learning in neural networks to properties of landscapes is explored in (Baldassi et al., 2016, 2015). This work is based on realization that in the simple model of binary perceptron learning dynamics ends in a part of the weight-space that has many low-loss close-by configurations. It goes on to suggest that learning favours these wide parts in the space of weights, and argues that this might explain why algorithms are attracted to wide local minima and why by doing so their generalization properties improve. An interesting spin-off of this theory is a variant of the stochastic gradient descent algorithm suggested in Chaudhari et al. 2016).

\section{E. Applications of ML in Statistical Physics}

When a researcher in theoretical physics encounters deep neural networks where the early layers are learning to represent the input data at a finer scale than the later layers, she immediately thinks about renormalization group as used in physics in order to extract macroscopic behaviour from microscopic rules. This analogy was explored for instance in (Bény, 2013, Mehta and Schwab, 2014). Analogies between renormalization group and the principle component analysis were reported in (Bradde and Bialek, 2017).

A natural idea is to use neural networks in order to learn new renormalization schemes. First attempts in this direction appeared in Koch-Janusz and Ringel, 2018, Li and Wang, 2018). However, it remains to be shown whether this can lead to new physical discoveries in models that were not well understood previously.

Phase transitions are boundaries between different phases of matter. They are usually determined using order parameters. In some systems it is not a priori clear how to determine the proper order parameter. A natural idea is that a neural networks may be able to learn appropriate order parameters and locate the phase transition without a priori physical knowledge. This idea was explored in (Carrasquilla and Melko, 2017, Morningstar and Melko, 2018; Tanaka and Tomiya, 2017a; Van Nieuwenburg et al. 2017) in a range of models using configurations sampled uniformly from the model of interest (obtained using Monte Carlo simulations) in different phases or at different temperatures and using supervised learning in order to classify the configurations to their phases. Extrapolating to configurations not used in the training set plausibly leads to determination of the phase transitions in the studied models. These general guiding principles have been used in a large number of applications to analyze both synthetic and experimental data. Specific cases in the context of many-body quantum physics are detailed in Section IV.C.

Detailed understanding of the limitations of these methods in terms of identifying previously unknown order parameters, as well as understanding whether they can reliably distinguish between a true thermodynamic phase transitions and a mere cross-over are yet to be clarified. Experiments presented on the Ising model in (Mehta et al. 2018) provide some preliminary thoughts in that direction. Some underlying mechanisms are discussed in (Kashiwa et al. 2019). Kernel based learning method for learning phases in frustrated magnetic materials that is more easily interpretable and able to identify complex order parameters is introduced and studied in (Greitemann et al., 2019, Liu et al., 2019).

Disordered and glassy solids where identifications of the order parameter is particularly challenging were also studied. In particular (Nussinov et al., 2016, Ronhovde et al., 2011) use multi-scale network clustering methods to identify spatial and spatio-temporal structures in glasses, (Cubuk et al., 2015) learn to identify structural flow defects, and (Schoenholz et al. 2017) argues to identify a parameter that captures the history dependence of the disordered system.

In an ongoing effort to go beyond the limitations of supervised learning to classify phases and identify phase transitions, several direction towards unsupervised learning are begin explored. For instance, in (Wetzel, 2017) for the Ising and XY model, in (Wang and Zhai, 2017, 2018) for frustrated spin systems. The work of (Martiniani et al., 2019) explores the direction of identifying phases from simple compression of the underlying configurations.

Machine learning also provides exciting set of tools to study, predict and control non-linear dynamical systems. For instance (Pathak et al. 2018,2017$)$ used recurrent neural networks called an echo state networks or reservoir computers (Jaeger and Haas, 2004) to predict the trajectories of a chaotic dynamical system and of models used for weather prediction. The authors of (Reddy et al., 2016, 2018) used reinforcement learning to teach an autonomous glider to literally soar like a bird, using thermals in the atmosphere.

\section{F. Outlook and Challenges}

The described methods of statistical physics are quite powerful in dealing with high-dimensional data sets and models. The largest difference between traditional learning theories and the theories coming from statistical physics is that the later are often based on toy generative models of data. This leads to solvable models in the sense that quantities of interest such as achievable errors can be computed in a closed form, including constant terms. This is in contrast with aims in the mainstream learning theory that aims to provide worst case bounds on error under general assumptions on the setting (data structure, or architecture). These two approaches are 
complementary and ideally will meet in the future once we understand what are the key conditions under which practical cases are close to worse cases, and what are the right models of realistic data and functions.

The next challenge for the statistical physics approach is to formulate and solve models that are in some kind of universality class of the real settings of interest. Meaning that they reproduce all important aspects of the behaviour that is observed in practical application of neural networks. For this we need to model the input data no longer as iid vectors, but for instance as outputs from a generative neural network as in (Gabrié et al. , 2018), or as perceptual manifolds as in (Chung et al. 2018). The teacher network that is producing the labels (in an supervised setting) needs to model suitably the correlation between the structure in the data and the label. We need to find out how to analyze the (stochastic) gradient descent algorithm and its relevant variants. Promising works in this direction, that rely of the dynamic meanfield theory of glasses are (Mannelli et al., 2018, 2019). We need to generalize the existing methodology to multilayer networks with extensive width of hidden layers.

Going back to the direction of using machine learning for physics, the full potential of ML in research of nonlinear dynamical systems and statistical physics is yet to be uncovered. The above mentioned works certainly provide an exciting appetizer.

\section{PARTICLE PHYSICS AND COSMOLOGY}

A diverse portfolio of on-going and planned experiments is well poised to explore the universe from the unimaginably small world of fundamental particles to the awe inspiring scale of the universe. Experiments like the Large Hadron Collider (LHC) and the Large Synoptic Survey Telescope (LSST) deliver enormous amounts of data to be compared to the predictions of specific theoretical models. Both areas have well established physical models that serve as null hypotheses: the standard model of particle physics and $\Lambda \mathrm{CDM}$ cosmology, which includes cold dark matter and a cosmological constant $\Lambda$. Interestingly, most alternate hypotheses considered are formulated in the same theoretical frameworks, namely quantum field theory and general relativity. Despite such sharp theoretical tools, the challenge is still daunting as the expected deviations from the null are expected to be incredibly tiny and revealing such subtle effects requires a robust treatment of complex experimental apparatuses. Complicating the statistical inference is that the most high-fidelity predictions for the data do not come in the from simple closed-form equations, but instead in complex computer simulations.

Machine learning is making waves in particle physics and cosmology as it offers a suit of techniques to confront these challenges and a new perspective that motivates bold new strategies. The excitement spans the theoretical and experimental aspects of these fields and includes both applications with immediate impact as well as the prospect of more transformational changes in the longer term.

\section{A. The role of the simulation}

An important aspect of the use of machine learning in particle physics and cosmology is the use of computer simulations to generate samples of labeled training data $\left\{X_{\mu}, y_{\mu}\right\}_{\mu=1}^{n}$. For example, when the target $y$ refers to a particle type, particular scattering process, or parameter appearing in the fundamental theory, it can often be specified directly in the simulation code so that the simulation directly samples $X \sim p(\cdot \mid y)$. In other cases, the simulation is not directly conditioned on $y$, but provides samples $(X, Z) \sim p(\cdot)$, where $Z$ are latent variables that describe what happened inside the simulation, but which are not observable in an actual experiment. If the target label can be computed from these latent variables via a function $y(Z)$, then labeled training data $\left\{X_{\mu}, y\left(Z_{\mu}\right)\right\}_{\mu=1}^{n}$ can also be created from the simulation. The use of highfidelity simulations to generate labeled training data has not only been the key to early successes of supervised learning in these areas, but also the focus of research addressing the shortcomings of this approach.

Particle physicists have developed a suite of highfidelity simulations that are hierarchically composed to describe interactions across a huge range of length scales. The components of these simulations include Feynman diagrammatic perturbative expansion of quantum field theory, phenomenological models for complex patterns of radiation, and detailed models for interaction of particles with matter in the detector. While the resulting simulation has high fidelity, the simulation itself has free parameters to be tuned and number of residual uncertainties in the simulation must be taken into account in down-stream analysis tasks.

Similarly, cosmologists can simulate the evolution of the universe at different length scales using general relativity and relevant non-gravitational effects of matter and radiation that becomes increasingly important during structure formation. There is a rich array of approximations that can be made in specific settings that provide enormous speedups compared to the computationally expensive $N$-body simulations of billions of massive objects that interact gravitationally, which become prohibitively expensive once non-gravitational feedback effects are included.

Cosmological simulations generally involve deterministic evolution of stochastic initial conditions due to primordial quantum fluctuations. The $N$-body simulations are very expensive, so there are relatively few simulations, but they cover a large space-time volume that is 
statistically isotropic and homogeneous at large scales. In contrast, particle physics simulations are stochastic throughout from the initial high-energy scattering to the low-energy interactions in the detector. Simulations for high-energy collider experiments can run on commodity hardware in a parallel manner, but the physics goals requires enormous numbers of simulated collisions.

Because of the critical role of the simulation in these fields, much of the recent research in machine learning is related to simulation in one way or another. These goals of these recent works are to:

- develop techniques that are more data efficient by incorporating domain knowledge directly into the machine learning models;

- incorporate the uncertainties in the simulation into the training procedure;

- develop weakly supervised procedures that can be applied to real data and do not rely on the simulation;

- develop anomaly detection algorithms to find anomalous features in the data without simulation of a specific signal hypothesis;

- improve the tuning of the simulation, reweight or adjust the simulated data to better match the real data, or use machine learning to model residuals between the simulation and the real data;

- learn fast neural network surrogates for the simulation that can be used to quickly generate synthetic data;

- develop approximate inference techniques that make efficiently use of the simulation; and

- learn fast neural network surrogates that can be used directly for statistical inference.

\section{B. Classification and regression in particle physics}

Machine learning techniques have been used for decades in experimental particle physics to aid particle identification and event selection, which can be seen as classification tasks. Machine learning has also been used for reconstruction, which can be seen as a regression task. Supervised learning is used to train a predictive model based on large number of labeled training samples $\left\{X_{\mu}, y_{\mu}\right\}_{\mu=1}^{n}$, where $X$ denotes the input data and $y$ the target label. In the case of particle identification, the input features $X$ characterize localized energy deposits in the detector and the label $y$ refers to one of a few particle species (e.g. electron, photon, pion, etc.). In the reconstruction task, the same type of sensor data $X$ are used, but the target label $y$ refers to the energy or momentum of the particle responsible for those energy deposits. These algorithms are applied to the bulk data processing of the LHC data.

Event selection, refers to the task of selecting a small subset of the collisions that are most relevant for a targeted analysis task. For instance, in the search for the Higgs boson, supersymmetry, and dark matter data analysts must select a small subset of the LHC data that is consistent with the features of these hypothetical "signal" processes. Typically these event selection requirements are also satisfied by so-called "background" processes that mimic the features of the signal either due to experimental limitations or fundamental quantum mechanical effects. Searches in their simplest form reduce to comparing the number of events in the data that satisfy these requirements to the predictions of a backgroundonly null hypothesis and signal-plus-background alternate hypothesis. Thus, the more effective the event selection requirements are at rejecting background processes and accept signal processes, the more powerful the resulting statistical analysis will be. Within high-energy physics, machine learning classification techniques have traditionally been referred to as multivariate analysis to emphasize the contrast to traditional techniques based on simple thresholding (or "cuts") applied to carefully selected or engineered features.

In the 1990s and early 2000s simple feed-forward neural networks were commonly used for these tasks. Neural networks were largely displaced by Boosted Decision Trees (BDTs) as the go-to for classification and regression tasks for more than a decade (Breiman et al. 1984 , Freund and Schapire, 1997; Roe et al., 2005). Starting around 2014, techniques based on deep learning emerged and were demonstrated to be significantly more powerful in several applications (for a recent review of the history, see Refs. (Guest et al. 2018; Radovic et al., 2018)).

Deep learning was first used for an event-selection task targeting hypothesized particles from theories beyond the standard model. It not only out-performed boosted decision trees, but also did not require engineered features to achieve this impressive performance (Baldi et al., 2014). In this proof-of-concept work, the network was a deep multi-layer perceptron trained with a very large training set using a simplified detector setup. Shortly after, the idea of a parametrized classifier was introduced in which the concept of a binary classifier was extended to a situation where the $y=1$ signal hypothesis is lifted to a composite hypothesis that is parameterized continuously, for instance, in terms of the mass of a hypothesized particle (Baldi et al. 2016b). 


\section{Jet Physics}

The most copious interactions at hadron colliders such as the LHC produce high energy quarks and gluons in the final state. These quarks and gluons radiate more quarks and gluons that eventually combine into colorneutral composite particles due to the phenomena of confinement. The resulting collimated spray of mesons and baryons that strike the detector is collectively referred to as a jet. Developing a useful characterization of the structure of a jet that are theoretically robust and that can be used to test the predictions of quantum chromodynamics (QCD) has been an active area of particle physics research for decades. Furthermore, many scenarios for physics Beyond the Standard Model predict the production of particles that decay into two or more jets. If those unstable particles are produced with a large momentum, then the resulting jets are boosted such that the jets overlap into a single fat jet with nontrivial substructure. Classifying these boosted or fat jets from the much more copiously produced jets from standard model processes involving quarks and gluons is an area that can significantly improve the physics reach of the LHC. More generally, identifying the progenitor for a jet is a classification task that is often referred to as jet tagging.

Shortly after the first applications of deep learning for event selection, deep convolutional networks were used for the purpose of jet tagging, where the low-level detector data lends itself to an image-like representation (Baldi et al., 2016a; de Oliveira et al., 2016). While machine learning techniques have been used within particle physics for decades, the practice has always been restricted to input features $X$ with a fixed dimensionality. One challenge in jet physics is that the natural representation of the data is in terms of particles, and the number of particles associated to a jet varies. The first application of a recurrent neural network in particle physics was in the context of flavor tagging (Guest et al. 2016). More recently, there has been an explosion of research into the use of different network architectures including recurrent networks operating on sequences, trees, and graphs (see Ref. (Larkoski et al. 2017) for a recent review for jet physics). This includes hybrid approaches that leverage domain knowledge in the design of the architecture. For example, motivated by techniques in natural language processing, recursive networks were designed that operate over tree-structures created from a class of jet clustering algorithms (Louppe et al. 2017a). Similarly, networks have been developed motivated by invariance to permutations on the particles presented to the network and stability to details of the radiation pattern of particles, (Komiske et al., 2018b, 2019). Recently, comparisons of the different approaches for specific benchmark problems have been organized (Kasieczka et al. 2019).

In addition to classification and regression, machine learning techniques have been used for density estima- tion and modeling smooth spectra where an analytical form is not well motivated and the simulation has significant uncertainties (Frate et al. 2017 ). The work also allows one to model alternative signal hypotheses with a diffuse prior instead of a specific concrete physical model. More abstractly, the Gaussian process in this work is being used to model the intensity of inhomogeneous Poisson point process, which is a scenario that is found in particle physics, astrophysics, and cosmology. One interesting aspect of this line of work is that the Gaussian process kernels can be constructed using compositional rules that correspond clearly to the causal model physicists intuitively use to describe the observation, which aids in interpretability (Duvenaud et al. 2013).

\section{Neutrino physics}

Neutrinos interact very feebly with matter, thus the experiments require large detector volumes to achieve appreciable interaction rates. Different types of interactions, whether they come from different species of neutrinos or background cosmic ray processes, leave different patterns of localized energy deposits in the detector volume. The detector volume is homogeneous, which motivates the use of convolutional neural networks.

The first application of a deep convolutional network in the analysis of data from a particle physics experiment was in the context of the $\mathrm{NO} \nu \mathrm{A}$ experiment, which uses scintillating mineral oil. Interactions in $\mathrm{NO} \nu \mathrm{A}$ lead to the production of light, which is imaged from two different vantage points. $\mathrm{NO} \nu \mathrm{A}$ developed a convolutional network that simultaneously processed these two images (Aurisano et al. 2016). Their network improves the efficiency (true positive rate) of selecting electron neutrinos by $40 \%$ for the same purity. This network has been used in searches for the appearance of electron neutrinos and for the hypothetical sterile neutrino.

Similarly, the MicroBooNE experiment detects neutrinos created at Fermilab. It uses 170 ton liquid-argon time projection chamber. Charged particles ionize the liquid argon and the ionization electrons drift through the volume to three wire planes. The resulting data is processed and represented by a 33-megapixel image, which is dominantly populated with noise and only very sparsely populated with legitimate energy deposits. The MicroBooNE collaboration used a FasterRCNN (Ren et al. 2015) to identify and localize neutrino interactions with bounding boxes (Acciarri et al. 2017). This success is important for future neutrino experiments based on liquid-argon time projection chambers, such as the Deep Underground Neutrino Experiment (DUNE).

In addition to the relatively low energy neutrinos produced at accelerator facilities, machine learning has also been used to study high-energy neutrinos with the IceCube observatory located at the south pole. In partic- 
ular, 3D convolutional and graph neural networks have been applied to a signal classification problem. In the latter approach, the detector array is modeled as a graph, where vertices are sensors and edges are a learned function of the sensors' spatial coordinates. The graph neural network was found to outperform both a traditionalphysics-based method as well as classical 3D convolutional neural network (Choma et al. 2018).

\section{Robustness to systematic uncertainties}

Experimental particle physicists are keenly aware that the simulation, while incredibly accurate, is not perfect. As a result, the community has developed a number of strategies falling roughly in two broad classes. The first involves incorporating the effect of mis-modeling when the simulation is used for training. This involves either propagating the underlying sources of uncertainty (e. g. calibrations, detector response, the quark and gluon composition of the proton, and the impact of higher-order corrections from perturbation theory, etc.) through the simulation and analysis chain. For each of these sources of uncertainty, a nuisance parameter $\nu$ is included, and the resulting statistical model $p(X \mid y, \nu)$ is parameterized by these nuisance parameters. In addition, the likelihood function for the data is augmented with a term $p(\nu)$ representing the uncertainty in these sources of uncertainty, as in the case of a penalized maximum likelihood analysis. In the context of machine learning, classifiers and regressors are typically trained using data generated from a nominal simulation $\nu=\nu_{0}$, yielding a predictive model $f\left(X \mid \nu_{0}\right)$. Treating this predictive model as fixed, it is possible to propagate the uncertainty in $\nu$ through $f\left(X \mid \nu_{0}\right)$ using the model $p(X \mid y, \nu) p(\nu)$. However, the down-stream statistical analysis based on this approach is not optimal since the predictive model was not trained taking into account the uncertainty on $\nu$.

In machine learning literature, this situation is often referred to as covariate shift between two domains represented by the training distribution $\nu_{0}$ and the target distribution $\nu$. Various techniques for domain adaptation exist to train classifiers that are robust to this change, but they tend to be restricted to binary domains $\nu \in\{$ train, target $\}$. To address this problem, an adversarial training technique was developed that extends domain adaptation to domains parametrized by $\nu \in \mathbb{R}^{q}$ (Louppe et al. 2016). The adversarial approach encourages the network to learn a pivotal quantity, where $p(f(X) \mid y, \nu)$ is independent of $\nu$, or equivalently $p(f(X), \nu \mid y)=p(f(X) \mid y) p(\nu)$. This adversarial approach has also been used in the context of algorithmic fairness, where one desires to train a classifiers or regressor that is independent of (or decorrelated with) specific continuous attributes or observable quantities. For instance, in jet physics one often would like a jet tag- ger that is independent of the jet invariant mass Shimmin et al. 2017). Previously, a different algorithm called uboost was developed to achieve similar goals for boosted decision trees (Rogozhnikov et al. , 2015; Stevens and Williams, 2013).

The second general strategy used within particle physics to cope with systematic mis-modeling in the simulation is to avoid using the simulation for modeling the distribution $p(X \mid y)$. In what follows, let $R$ denote an index over various subsets of the data satisfying corresponding selection requirements. Various data-driven strategies have been developed to relate distributions of the data in control regions, $p(X \mid y, R=0)$, to distributions in regions of interest, $p(X \mid y, R=1)$. These relationships also involve the simulation, but the art of this approach is to base those relationships on aspects of the simulation that are considered robust. The simplest example is estimating the distribution $p(X \mid y, R=1)$ for a specific process $y$ by identifying a subset of the data $R=0$ that is dominated by $y$ and $p(y \mid R=0) \approx 1$. This is an extreme situation that is limited in applicability.

Recently, weakly supervised techniques have been developed that only involve identifying regions where only the class proportions are known or assuming that the relative probabilities $p(y \mid R)$ are not linearly dependent (Komiske et al., 2018a, Metodiev et al., 2017) . The techniques also assume that the distributions $p(X \mid y, R)$ are independent of $R$, which is reasonable in some contexts and questionable in others. The approach has been used to train jet taggers that discriminate between quarks and gluons, which is an area where the fidelity of the simulation is no longer adequate and the assumptions for this method are reasonable. This weakly-supervised, data-driven approach is a major development for machine learning for particle physics, though it is limited to a subset of problems. For example, this approach is not applicable if one of the target categories $y$ corresponds to a hypothetical particle that may not exist or be present in the data.

\section{Triggering}

Enormous amounts of data must be collected by collider experiments such as the LHC, because the phenomena being targeted are exceedingly rare. The bulk of the collisions involve phenomena that have previously been studied and characterized, and the data volume associated with the full data stream is impractically large. As a result, collider experiments use a real-time datareduction system referred to as a trigger. The trigger makes the critical decision of which events to keep for future analysis and which events to discard. The ATLAS and CMS experiments retain only about 1 out of every 100,000 events. Machine learning techniques are used to various degrees in these systems. Essentially, the 
same particle identification (classification) tasks appears in this context, though the computational demands and performance in terms of false positives and negatives are different in the real-time environment.

The $\mathrm{LHCb}$ experiment has been a leader in using machine learning techniques in the trigger. Roughly $70 \%$ of the data selected by the LHC trigger is selected by machine learning algorithms. Initially, the experiment used a boosted decision tree for this purpose (Gligorov and Williams, 2013), which was later replaced by the MatrixNet algorithm developed by Yandex (Likhomanenko et al. 2015).

The Trigger systems often use specialized hardware and firmware, such as field-programmable gate arrays (FPGAs). Recently, tools have been developed to streamline the compilation of machine learning models for FPGAs to target the requirements of these real-time triggering systems (Duarte et al., 2018, Tsaris et al., 2018).

\section{Theoretical particle physics}

While the bulk of machine learning in particle physics and cosmology are focused on analysis of observational data, there are also examples of using machine learning as a tool in theoretical physics. For instance, machine learning has been used to characterize the landscape of string theories (Carifio et al. 2017), to identify the phase transitions of quantum chromodynamics (QCD) (Pang et al. 2018), and to study the the AdS/CFT correspondence (Hashimoto et al. 2018a b). Some of this work is more closely connected to the use of machine learning as a tool in condensed matter or many-body quantum physics. Specifically, deep learning has been used in the context of lattice QCD (LQCD). In an exploratory work in this direction, deep neural networks were used to predict the parameters in the QCD Lagrangian from lattice configurations (Shanahan et al. 2018). This is needed for a number of multi-scale action-matching approaches, which aim to improve the efficiency of the computationally intensive LQCD calculations. This problem was setup as a regression task, and one of the challenges is that there are relatively few training examples. Additionally, machine learning techniques are being used to reduce the autocorrelation time in the Markov Chains (Albergo et al. 2019, Tanaka and Tomiya, 2017b) In order to solve this task with few training examples it is important to leverage the known space-time and local gauge symmetries in the lattice data. Data augmentation is not a scalable solution given the richness of the symmetries. Instead the authors performed feature engineering that imposed gauge symmetry and space-time translational invariance. While this approach proved effective, it would be desirable to consider a richer class of networks, that are equivariant (or covariant) to the symmetries in the data (such approaches are discussed in Sec. III.F. A continuation of this work is being supported by the Argon Leadership Computing Facility. The a new Intel-Cray system Aurora, will be capable of over 1 exaflops and specifically is aiming at problems that combine traditional high performance computing with modern machine learning techniques.

\section{Classification and regression in cosmology}

\section{Photometric Redshift}

Due to the expansion of the universe the distant luminous objects are redshifted, and the distance-redshift relation is a fundamental component of observational cosmology. Very precise redshift estimates can be obtained through spectroscopy; however, such spectroscopic surveys are expensive and time consuming. Photometric surveys based on broadband photometry or imaging in a few color bands give a coarse approximation to the spectral energy distribution. Photometric redshift refers to the regression task of estimating redshifts from photometric data. In this case, the ground truth training data comes from precise spectroscopic surveys.

The traditional approaches to photometric redshift is based on template fitting methods (Benítez, 2000 Brammer et al., 2008, Feldmann et al., 2006). For more than a decade cosmologists have also used machine learning methods based on neural networks and boosted decision trees for photometric redshift (Carrasco Kind and Brunner, 2013, Collister and Lahav, 2004; Firth et al., 2003). One interesting aspect of this body of work is the effort that has been placed to go beyond a point estimate for the redshift. Various approaches exist to determine the uncertainty on the redshift estimate and to obtain a posterior distribution.

While the training data are not generated from a simulation, there is still a concern that the distribution of the training data may not be representative of the distribution of data that the models will be applied to. This type of covariate shift results from various selection effects in the spectroscopic survey and subtleties in the photometric surveys. The Dark Energy Survey considered a number of these approaches and established a validation process to evaluate them critically (Bonnett et al. 2016). Recently there has been work to use hierarchical models to build in additional causal structure in the models to be robust to these differences. In the language of machine learning, these new models aid in transfer learning and domain adaptation. The hierarchical models also aim to combine the interpretability of traditional template fitting approaches and the flexibility of the machine learning models (Leistedt et al., 2018). 


\section{Gravitational lens finding and parameter estimation}

One of the most striking effects of general relativity is gravitatioanl lensing, in which a massive foreground object warps the image of a background object. Strong gravitational lensing occurs, for example, when a massive foreground galaxy is nearly coincident on the sky with a background source. These events are a powerful probe of the dark matter distribution of massive galaxies and can provide valuable cosmological constraints. However, these systems are rare, thus a scalable and reliable lens finding system is essential to cope with large surveys such as LSST, Euclid, and WFIRST. Simple feedfoward, convolutional and residual neural networks (ResNets) have been applied to this supervised classification problem (Estrada et al., 2007 , Lanusse et al. 2018, Marshall et al. . 2009). In this setting, the training data came from simulation using PICS (Pipeline for Images of Cosmological Strong) lensing (Li et al., 2016) for the strong lensing ray-tracing and LensPop (Collett, 2015) for mock LSST observing. Once identified, characterizing the lensing object through maximum likelihood estimation is a computationally intensive non-linear optimization task. Recently, convolutional networks have been used to quickly estimate the parameters of the Singular Isothermal Ellipsoid density profile, commonly used to model strong lensing systems (Hezaveh et al., 2017).

\section{Other examples}

In addition to the examples above, in which the ground truth for an object is relatively unambiguous with a more labor-intensive approach, cosmologists are also leveraging machine learning to infer quantities that involve unobservable latent processes or the parameters of the fundamental cosmological model.

For example, 3D convolutional networks have been trained to predict fundamental cosmological parameters based on the dark matter spatial distribution (Ravanbakhsh et al., 2017) (see Fig. 1p. In this proof-of-concept work, the networks were trained using computationally intensive $N$-body simulations for the evolution of dark matter in the universe assuming specific values for the 10 parameters in the standard $\Lambda$ CDM cosmology model. In real applications of this technique to visible matter, one would need to model the bias and variance of the visible tracers with respect to the underlying dark matter distribution. In order to close this gap, convolutional networks have been trained to learn a fast mapping between the dark matter and visible galaxies (Zhang et al., 2019 ), allowing for a trade-off between simulation accuracy and computational cost. One challenge of this work, which is common to applications in solid state physics, lattice field theory, and many body quantum systems, is that the simulations are computationally expensive and thus there are relatively few statistically independent realizations of the large simulations $X_{\mu}$. As deep learning tends to require large labeled training data-sets, various types of subsampling and data augmentation approaches have been explored to ameliorate the situation. An alternative approach to subsampling is the so-called Backdrop, which provides stochastic gradients of the loss function even on individual samples by introducing a stochastic masking in the backpropagation pipeline (Golkar and Cranmer, 2018).

Inference on the fundamental cosmological model also appears in a classification setting. In particular, modified gravity models with massive neutrinos can mimic the predictions for weak-lensing observables predicted by the standard $\Lambda \mathrm{CDM}$ model. The degeneracies that exist when restricting the $X_{\mu}$ to second-order statistics can be broken by incorporating higher-order statistics or other rich representations of the weak lensing signal. In particular, the authors of (Peel et al. 2018) constructed a novel representation of the wavelet decomposition of the weak lensing signal as input to a convolutional network. The resulting approach was able to discriminate between previously degenerate models with $83 \%-100 \%$ accuracy.

Deep learning has also been used to estimate the mass of galaxy clusters, which are the largest gravitationally bound structures in the universe and a powerful cosmological probe. Much of the mass of these galaxy clusters comes in the form of dark matter, which is not directly observable. Galaxy cluster masses can be estimated via gravitational lensing, X-ray observations of the intracluster medium, or through dynamical analysis of the cluster's galaxies. The first use of machine learning for a dynamical cluster mass estimate was performed using Support Distribution Machines (Póczos et al., 2012) on a dark-matter-only simulation (Ntampaka et al., 2015 . 2016). A number of non-neural network algorithms including Gaussian process regression (kernel ridge regression), support vector machines, gradient boosted tree regressors, and others have been applied to this problem using the MACSIS simulations (Henson et al., 2016) for training data. This simulation goes beyond the darkmatter-only simulations and incorporates the impact of various astrophysical processes and allows for the development of a realistic processing pipeline that can be applied to observational data. The need for an accurate, automated mass estimation pipeline is motivated by large surveys such as eBOSS, DESI, eROSITA, SPT-3G, ActPol, and Euclid. The authors found that compared to the traditional $\sigma-M$ relation the predicted-to-true mass ratio using machine learning techniques is reduced by a factor of 4 (Armitage et al. 2019). Most recently, convolutional neural networks have been used to mitigate systematics in the virial scaling relation, further improving dynamical mass estimates (Ho et al. 2019 ). Convolutional neural networks have also been used to estimate cluster masses with synthetic (mock) X-ray observations 


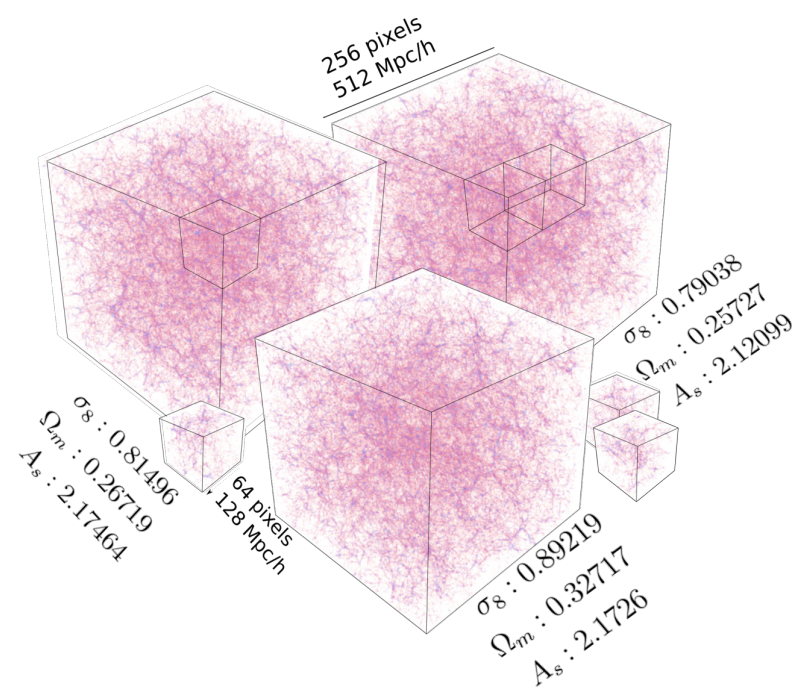

Figure 1 Dark matter distribution in three cubes produced using different sets of parameters. Each cube is divided into small sub- cubes for training and prediction. Note that although cubes in this figure are produced using very different cosmological parameters in our constrained sampled set, the effect is not visually discernible. Reproduced from (Ravanbakhsh et al. 2017).

of galaxy clusters, where the authors find the scatter in the predicted mass is reduced compared to traditional Xray luminosity based methods (Ntampaka et al. 2018).

\section{Inverse Problems and Likelihood-free inference}

As stressed repeatedly, both particle physics and cosmology are characterized by well motivated, high-fidelity forward simulations. These forward simulations are either intrinsically stochastic - as in the case of the probabilistic decays and interactions found in particles physics simulations - or they are deterministic - as in the case of gravitational lensing or N-body gravitational simulations. However, even deterministic physics simulators usually are followed by a probabilistic description of the observation based on Poisson counts or a model for instrumental noise. In both cases, one can consider the simulation as implicitly defining the distribution $p(X, Z \mid y)$, where $X$ refers to the observed data, $Z$ are unobserved latent variables that take on random values inside the simulation, and $y$ are parameters of the forward model such as coefficients in a Lagrangian or the 10 parameters of $\Lambda \mathrm{CDM}$ cosmology. Many scientific tasks can be characterized as inverse problems where one wishes to infer $Z$ or $y$ from $X=x$. The simplest cases that we have considered are classification where $y$ takes on categorical values and regression where $y \in \mathbb{R}^{d}$. The point estimates $\hat{y}(X=x)$ and $\hat{Z}(X=x)$ are useful, but in scientific applications we often require uncertainty on the estimate.

In many cases, the solution to the inverse problem is ill-posed, in the sense that small changes in $X$ lead to large changes in the estimate. This implies the estimator will have high variance. In some cases the forward model is equivalent to a linear operator and the maximum likelihood estimate $\hat{y}_{\mathrm{MLE}}(X)$ or $\hat{Z}_{\mathrm{MLE}}(X)$ can be expressed as a matrix inversion. In that case, the instability of the inverse is related to the matrix for the forward model being poorly conditioned. While the maximum likelihood estimate may be unbiased, it tends to be high variance. Penalized maximum likelihood, ridge regression (Tikhonov regularization), and Gaussian process regression are closely related approaches to the bias-variance trade-off.

Within particle physics, this type of problem is often referred to as unfolding. In that case, one is often interested in the distribution of some kinematic property of the collisions prior to the detector effects, and $X$ represents a smeared version of this quantity after folding in the detector effects. Similarly, estimating the parton density functions that describe quarks and gluons inside the proton can be cast as an inverse problem of this sort (Ball et al. 2015, Forte et al. 2002). Recently, both neural networks and Gaussian processes with more sophisticated, physically inspired kernels have been applied to these problems (Bozson et al., 2018; Frate et al., 2017). In the context of cosmology, an example inverse problem is to denoise the Laser Interferometer Gravitational-Wave Observatory (LIGO) time series to the underlying waveform from a gravitational wave (Shen et al., 2019). Generative Adversarial Networks (GANs) have even been used in the context of inverse problems where they were used to denoise and recover images of galaxies beyond naive deconvolution limits (Schawinski et al. 2017$)$. Another example involves estimating the image of a background object prior to being gravitationally lensed by a foreground object. In this case, describing a physically motivated prior for the background object is difficult. Recently, recurrent 
inference machines (Putzky and Welling, 2017) have been introduced as way to implicitly learn a prior for such inverse problems, and they have successfully been applied to strong gravitational lensing (Morningstar et al. 2018 , 2019).

A more ambitious approach to inverse problems involves providing detailed probabilistic characterization of $y$ given $X$. In the frequentist paradigm one would aim to characterize the likelihood function $L(y)=p(X=x \mid y)$, while in a Bayesian formalism one would wish to characterize the posterior $p(y \mid X=x) \propto p(X=x \mid y) p(y)$. The analogous situation happens for inference of latent variables $Z$ given $X$. Both particle physics and cosmology have well-developed approaches to statistical inference based on detailed modeling of the likelihood, Markov Chain Monte Carlo (MCMC) (Foreman-Mackey et al. 2013), Hamiltonian Monte Carlo, and variational inference (Jain et al., 2018; Lang et al., 2016, Regier et al., 2018). However, all of these approaches require that the likelihood function is tractable.

\section{Likelihood-free Inference}

Somewhat surprisingly, the probability density, or likelihood, $p(X=x \mid y)$ that is implicitly defined by the simulator is often intractable. Symbolically, the probability density can be written $p(X \mid y)=\int p(X, Z \mid y) d Z$, where $Z$ are the latent variables of the simulation. The latent space of state-of-the-art simulations is enormous and highly structured, so this integral cannot be performed analytically. In simulations of a single collision at the LHC, $Z$ may have hundreds of millions of components. In practice, the simulations are often based on Monte Carlo techniques and generate samples $\left(X_{\mu}, Z_{\mu}\right) \sim p(X, Z \mid y)$ from which the density can be estimated. The challenge is that if $X$ is high-dimensional it is difficult to accurately estimate those densities. For example, naive histogrambased approaches do not scale to high dimensions and kernel density estimation techniques are only trustworthy to around 5-dimensions. Adding to the challenge is that the distributions have a large dynamic range, and the interesting physics often sits in the tails of the distributions.

The intractability of the likelihood implicitly defined by the simulations is a foundational problem not only for particle physics and cosmology, but many other areas of science as well including epidemiology and phylogenetics. This has motivated the development of so-called likelihood-free inference algorithms, which only require the ability to generate samples from the simulation in the forward mode.

One prominent technique, is Approximate Bayesian Computation $(\mathrm{ABC})$. In $\mathrm{ABC}$ one performs Bayesian inference using MCMC or a rejection sampling approach in which the likelihood is approximated by the proba- bility $p(\rho(X, x)<\epsilon)$, where $x$ is the observed data to be conditioned on, $\rho\left(x^{\prime}, x\right)$ is some distance metric between $x$ and the output of the simulator $x^{\prime}$, and $\epsilon$ is a tolerance parameter. As $\epsilon \rightarrow 0$, one recovers exact Bayesian inference; however, the efficiency of the procedure vanishes. One of the challenges for ABC, particularly for high-dimensional $x$, is the specification of the distance measure $\rho\left(x^{\prime}, x\right)$ that maintains reasonable acceptance efficiency without degrading the quality of the inference (Beaumont et al., 2002, Marin et al., 2012, Marjoram et al., 2003; Sisson and Fan, 2011; Sisson et al., 2007). This approach to estimating the likelihood is quite similar to the traditional practice in particle physics of using histograms or kernel density estimation to approximate $\hat{p}(x \mid y) \approx p(x \mid y)$. In both cases, domain knowledge is required to identify useful summary in order to reduce the dimensionality of the data. An interesting extension of the $\mathrm{ABC}$ technique utilizes universal probabilistic programming. In particular, a technique known as inference compilation is a sophisticated form of importance sampling in which a neural network controls the random number generation in the probabilistic program to bias the simulation to produce outputs $x^{\prime}$ closer to the observed $x$ (Le et al. 2017).

The term $\mathrm{ABC}$ is often used synonymously with the more general term likelihood-free inference; however, there are a number of other approaches that involve learning an approximate likelihood or likelihood ratio that is used as a surrogate for the intractable likelihood (ratio). For example, neural density estimation with autoregressive models and normalizing flows (Larochelle and Murray, 2011; Papamakarios et al., 2017, Rezende and Mohamed, 2015) have been used for this purpose and scale to higher dimensional data (Cranmer and Louppe, 2016, Papamakarios et al. 2018). Alternatively, training a classifier to discriminate between $x \sim p(x \mid y)$ and $x \sim p\left(x \mid y^{\prime}\right)$ can be used to estimate the likelihood ratio $\hat{r}\left(x \mid y, y^{\prime}\right) \approx p(x \mid y) / p\left(x \mid y^{\prime}\right)$, which can be used for inference in either the frequentist or Bayesian paradigm (Brehmer et al., 2018c; Cranmer et al., 2015, Hermans et al. 2019).

\section{Examples in particle physics}

Thousands of published results within particle physics, including the discovery of the Higgs boson, involve statistical inference based on a surrogate likelihood $\hat{p}(x \mid y)$ constructed with density estimation techniques applied to synthetic datasets generated from the simulation. These typically are restricted to one- or two-dimensional summary statistics or no features at all other than the number of events observed. While the term likelihood-free inference is relatively new, it is core to the methodology of experimental particle physics.

More recently, a suite of likelihood-free inference tech- 


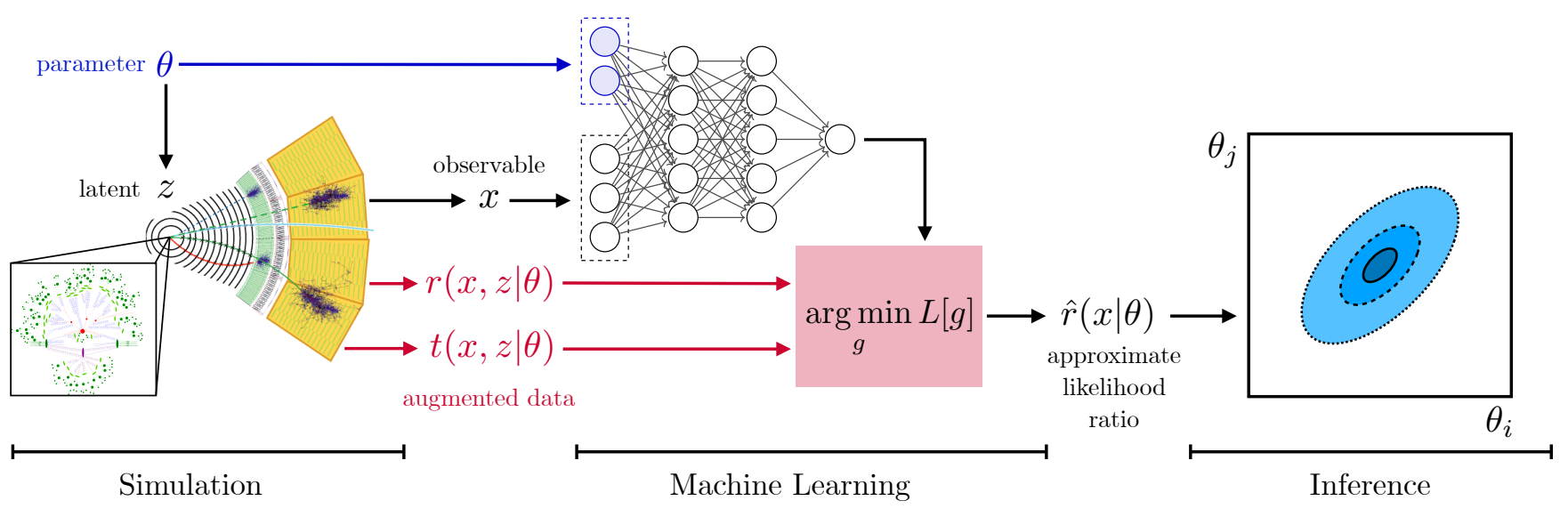

Figure 2 A schematic of machine learning based approaches to likelihood-free inference in which the simulation provides training data for a neural network that is subsequently used as a surrogate for the intractable likelihood during inference. Reproduced from (Brehmer et al. 2018b).

niques based on neural networks have been developed and applied to models for physics beyond the standard model expressed in terms of effective field theory (EFT) (Brehmer et al. 2018a b). EFTs provide a systematic expansion of the theory around the standard model that is parametrized by coefficients for quantum mechanical operators, which play the role of $y$ in this setting. One interesting observation in this work is that even though the likelihood and likelihood ratio are intractable, the joint likelihood ratio $r\left(x, z \mid y, y^{\prime}\right)$ and the joint score $t(x, z \mid y)=\nabla_{y} \log p(x, z \mid y)$ are tractable and can be used to augment the training data (see Fig. 2) and dramatically improve the sample efficiency of these techniques (Brehmer et al. 2018c).

In addition, an inference compilation technique has been applied to inference of a tau-lepton decay. This proof-of-concept effort required developing probabilistic programming protocol that can be integrated into existing domain-specific simulation codes such as SHERPA and GEANT4 (Baydin et al., 2018, Casado et al., 2017). This approach provides Bayesian inference on the latent variables $p(Z \mid X=x)$ and deep interpretability as the posterior corresponds to a distribution over complete stacktraces of the simulation, allowing any aspect of the simulation to be inspected probabilistically.

Another technique for likelihood-free inference that was motivated by the challenges of particle physics is known as adversarial variational optimization (AVO) (Louppe et al. 2017b). AVO parallels generative adversarial networks, where the generative model is no longer a neural network, but instead the domain-specific simulation. Instead of optimizing the parameters of the network, the goal is to optimize the parameters of the simulation so that the generated data matches the target data distribution. The main challenge is that, unlike neural networks, most scientific simulators are not differentiable. To get around this problem, a variational optimization technique is used, which provides a differentiable surrogate loss function. This technique is being investigated for tuning the parameters of the simulation, which is a computationally intensive task in which Bayesian optimization has also recently been used (Ilten et al. 2017).

\section{Examples in Cosmology}

Within Cosmology, early uses of ABC include constraining thick disk formation scenario of the Milky Way (Robin et al., 2014) and inferences on rate of morphological transformation of galaxies at high redshift (Cameron and Pettitt, 2012), which aimed to track the Hubble parameter evolution from type Ia supernova measurements. These experiences motivated the development of tools such as CosmoABC to streamline the application of the methodology in cosmological applications (Ishida et al. 2015).

More recently, likelihood-free inference methods based on machine learning have also been developed motivated by the experiences in cosmology. To confront the challenges of $\mathrm{ABC}$ for high-dimensional observations $X$, a data compression strategy was developed that learns summary statistics, that maximize the Fisher information on the parameters (Alsing et al., 2018, Charnock et al. 2018). The learned summary statistics approximate the sufficient statistics for the implicit likelihood in a small neighborhood of some nominal or fiducial parameter value. This approach is closely connected to that of (Brehmer et al., 2018c). Recently, these approaches have been extended to learn summary statistics that are robust to systematic uncertainties (Alsing and Wandelt, 2019). 


\section{E. Generative Models}

An active area in machine learning research involves using unsupervised learning to train a generative model to produce a distribution that matches some empirical distribution. This includes generative adversarial networks (GANs) (Goodfellow et al., 2014), variational autoencoders (VAEs) (Kingma and Welling, 2013; Rezende et al., 2014), autoregressive models, and models based on normalizing flows (Larochelle and Murray, 2011; $\mathrm{Pa}$ pamakarios et al., 2017; Rezende and Mohamed, 2015).

Interestingly, the same issue that motivates likelihoodfree inference, the intractability of the density implicitly defined by the simulator also appears in generative adversarial networks (GANs). If the density of a GAN were tractable, GANs would be trained via standard maximum likelihood, but because their density is intractable a trick was needed. The trick is to introduce an adversary - i.e. the discriminator network used to classify the samples from the generative model and samples taken from the target distribution. The discriminator is effectively estimating the likelihood ratio between the two distributions, which provides a direct connections to the approaches to likelihood-free inference based on classifiers (Cranmer and Louppe, 2016.

Operationally, these models play a similar role as traditional scientific simulators, though traditional simulation codes also provide a causal model for the underlying data generation process grounded in physical principles. However, traditional scientific simulators are often very slow as the distributions of interest emerge from a lowlevel microphysical description. For example, simulating collisions at the LHC involves atomic-level physics of ionization and scintillation. Similarly, simulations in cosmology involve gravitational interactions among enormous numbers of massive objects and may also include complex feedback processes that involve radiation, star formation, etc. Therefore, learning a fast approximation to these simulations is of great value.

Within particle physics early work in this direction included GANs for energy deposits from particles in calorimeters (Paganini et al. 2018a b), which is being studied by the ATLAS collaboration (ATLAS Collaboration, 2018). In Cosmology, generative models have been used to learn the simulation for cosmological structure formation (Rodríguez et al. 2018). In an interesting hybrid approach, a deep neural network was used to predict the non-linear structure formation of the universe from as a residual from a fast physical simulation based on linear perturbation theory (He et al. 2018).

In other cases, well-motivated simulations do not always exist or are impractical. Nevertheless, having a generative model for such data can be valuable for the purpose of calibration. An illustrative example in this direction comes from (Ravanbakhsh et al. 2016), see Fig. 3. The authors point out that the next generation of cos- mological surveys for weak gravitational lensing rely on accurate measurements of the apparent shapes of distant galaxies. However, shape measurement methods require a precise calibration to meet the accuracy requirements of the science analysis. This calibration process is challenging as it requires large sets of high quality galaxy images, which are expensive to collect. Therefore, the GAN enables an implicit generalization of the parametric bootstrap.

\section{F. Outlook and Challenges}

While particle physics and cosmology have a long history in utilizing machine learning methods, the scope of topics that machine learning is being applied to has grown significantly. Machine learning is now seen as a key strategy to confronting the challenges of the upgraded High-Luminosity LHC (Albertsson et al., 2018; Apollinari et al. , 2015) and is influencing the strategies for future experiments in both cosmology and particle physics (Ntampaka et al. 2019). One area in particular that has gathered a great deal of attention at the LHC is the challenge of identifying the tracks left by charged particles in high-luminostiy environments (Farrell et al. 2018), which has been the focus of a recent kaggle challenge.

In almost all areas where machine learning is being applied to physics problems, there is a desire to incorporate domain knowledge in the form of hierarchical structure, compositional structure, geometrical structure, or symmetries that are known to exist in the data or the datageneration process. Recently, there has been a spate of work from the machine learning community in this direction (Bronstein et al., 2017, Cohen and Welling, 2016, Cohen et al., 2018; Cohen et al., 2019, Kondor, 2018, Kondor et al., 2018; Kondor and Trivedi, 2018). These developments are being followed closely by physicists, and already being incorporated into contemporary research in this area.

\section{MANY-BODY QUANTUM MATTER}

The intrinsic probabilistic nature of quantum mechanics makes physical systems in this realm an effectively infinite source of big data, and a very appealing playground for ML applications. Paradigmatic example of this probabilistic nature is the measurement process in quantum physics. Measuring the position $\mathbf{r}$ of an electron orbiting around the nucleus can only be approximately inferred from measurements. An infinitely precise classical measurement device can only be used to record the outcome of a specific observation of the electron position. Ultimately, a complete characterization of the measurement process is given by the wave function 


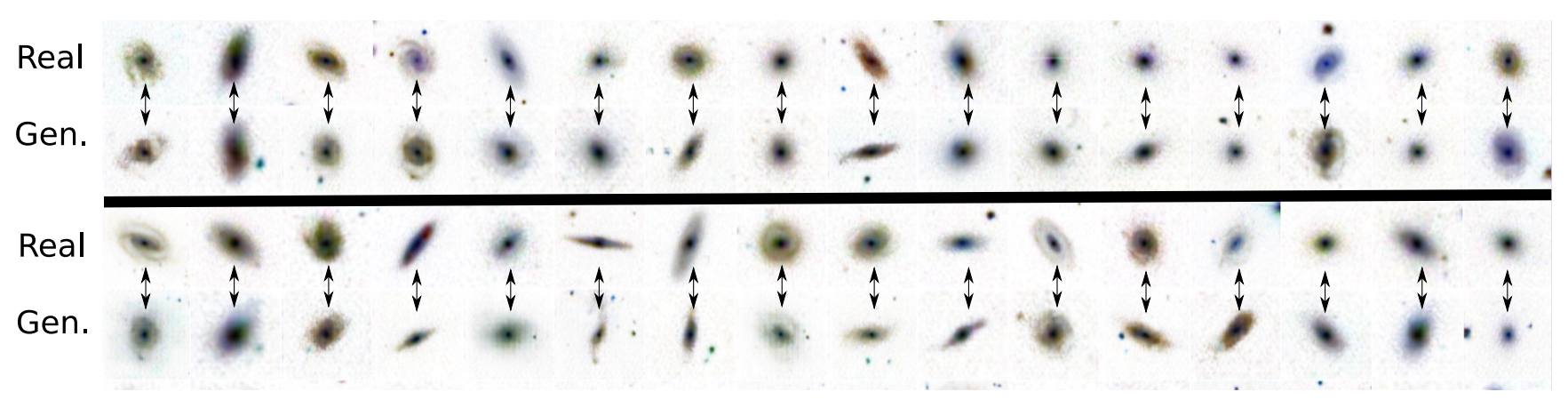

Figure 3 Samples from the GALAXY-ZOO dataset versus generated samples using conditional generative adversarial network. Each synthetic image is a $128 \times 128$ colored image (here inverted) produced by conditioning on a set of features $y \in[0,1]^{37}$. The pair of observed and generated images in each column correspond to the same $y$ value. Reproduced from (Ravanbakhsh et al. 2016).

$\Psi(\mathbf{r})$, whose square modulus ultimately defines the probability $P(\mathbf{r})=|\Psi(\mathbf{r})|^{2}$ of observing the electron at a given position in space. While in the case of a single electron both theoretical predictions and experimental inference for $P(\mathbf{r})$ are efficiently performed, the situation becomes dramatically more complex in the case of many quantum particles. For example, the probability of observing the positions of $N$ electrons $P\left(\mathbf{r}_{1}, \ldots \mathbf{r}_{N}\right)$ is an intrinsically high-dimensional function, that can seldom be exactly determined for $N$ much larger than a few tens. The exponential hardness in estimating $P\left(\mathbf{r}_{1}, \ldots \mathbf{r}_{N}\right)$ is itself a direct consequence of estimating the complex-valued manybody amplitudes $\Psi\left(\mathbf{r}_{1} \ldots \mathbf{r}_{N}\right)$ and is commonly referred to as the quantum many-body problem. The quantum many-body problem manifests itself in a variety of cases. These most chiefly include the theoretical modeling and simulation of complex quantum systems - most materials and molecules - for which only approximate solutions are often available. Other very important manifestations of the quantum many-body problem include the understanding and analysis of experimental outcomes, especially in relation with complex phases of matter. In the following, we discuss some of the ML applications focused on alleviating some of the challenging theoretical and experimental problems posed by the quantum many-body problem.

\section{A. Neural-Network quantum states}

Neural-network quantum states (NQS) are a representation of the many-body wave-function in terms of artificial neural networks (ANNs) (Carleo and Troyer, 2017). A commonly adopted choice is to parameterize wavefunction amplitudes as a feed-forward neural network:

$$
\Psi(\mathbf{r})=g^{(L)}\left(W^{(L)} \ldots g^{(2)}\left(W^{(2)} g^{(1)}\left(W^{(1)} \mathbf{r}\right)\right)\right),
$$

with similar notation to what introduced in Eq. (2).

Early works have mostly concentrated on shallow networks, and most notably Restricted Boltzmann Machines
(RBM) (Smolensky, 1986). RBMs with hidden unit in $\{ \pm 1\}$ and without biases on the visible units formally correspond to FFNNs of depth $L=2$, and activations $g^{(1)}(x)=\log \cosh (x), g^{(2)}(x)=\exp (x)$. An important difference with respect to RBM applications for unsupervised learning of probability distributions, is that when used as NQS RBM states are typically taken to have complex-valued weights (Carleo and Troyer, 2017). Deeper architectures have been consistently studied and introduced in more recent work, for example NQS based on fully-connected, and convolutional deep networks (Choo et al., 2018; Saito, 2018, Sharir et al., 2019), see Fig. 4 for a schematic example. A motivation to use deep FFNN networks, apart from the practical success of deep learning in industrial applications, also comes from more general theoretical arguments in quantum physics. For example, it has been shown that deep NQS can sustain entanglement more efficiently than RBM states (Levine et al. 2019, Liu et al., 2017a). Other extensions of the NQS representation concern representation of mixed states described by density matrices, rather than pure wave-functions. In this context, it is possible to define positive-definite RBM parametrizations of the density matrix (Torlai and Melko, 2018).

One of the specific challenges emerging in the quantum domain is imposing physical symmetries in the NQS representations. In the case of a periodic arrangement of matter, spatial symmetries can be imposed using convolutional architectures similar to what is used in image classification tasks (Choo et al., 2018, Saito, 2018, Sharir et al., 2019). Selecting high-energy states in different symmetry sectors has also been demonstrated (Choo et al., 2018). While spatial symmetries have analogous counterparts in other ML applications, satisfying more involved quantum symmetries often needs a deep rethinking of ANN architectures. The most notable case in this sense is the exchange symmetry. For bosons, this amounts to imposing the wave-function to be permutationally invariant with respect to exchange of particle 

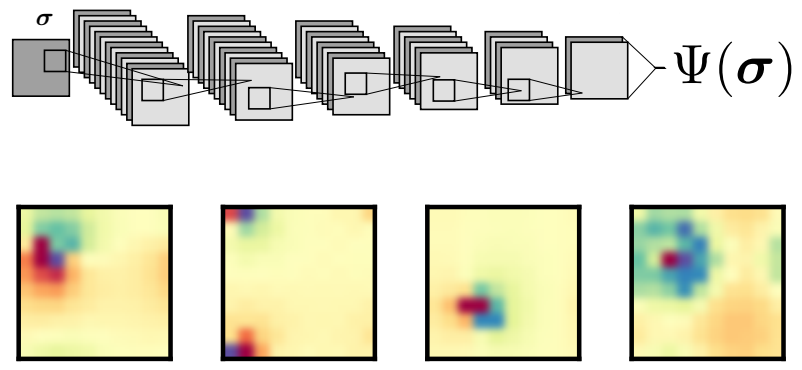

Figure 4 (Top) Example of a shallow convolutional neural network used to represent the many-body wave-function of a system of spin 1/2 particles on a square lattice. (Bottom) Filters of a fully-connected convolutional RBM found in the variational learning of the ground-state of the two-dimensional Heisenberg model, adapted from (Carleo and Troyer 2017).

indices. The Bose-Hubbard model has been adopted as a benchmark for ANN bosonic architectures, with state-ofthe-art results having been obtained (Saito, 2017, 2018, Saito and Kato, 2017; Teng, 2018). The most challenging symmetry is, however, certainly the fermionic one. In this case, the NQS representation needs to encode the antisymmetry of the wave-function (exchanging two particle positions, for example, leads to a minus sign). In this case, different approaches have been explored, mostly expanding on existing variational ansatz for fermions. A symmetric RBM wave-function correcting an antisymmetric correlator part has been used to study two-dimensional interacting lattice fermions (Nomura et al., 2017). Other approaches have tackled the fermionic symmetry problem using a backflow transformation of Slater determinants (Luo and Clark, 2018), or directly working in first quantization (Han et al. |2018a). The situation for fermions is certainly the most challenging for ML approaches at the moment, owing to the specific nature of the symmetry. On the applications side, NQS representations have been used so-far along three main different research lines.

\section{Representation theory}

An active area of research concerns the general expressive power of NQS, as also compared to other families of variational states. Theoretical activity on the representation properties of NQS seeks to understand how large, and how deep should be neural networks describing interesting interacting quantum systems. In connection with the first numerical results obtained with RBM states, the entanglement has been soon identified as a possible candidate for the expressive power of NQS. RBM states for example can efficiently support volume-law scaling (Deng et al. 2017b), with a number of variational parameters scaling only polynomially with system size. In this direction, the language of tensor networks has been par- ticularly helpful in clarifying some of the properties of NQS (Chen et al., 2018b; Pastori et al., 2018). A family of NQS based on RBM states has been shown to be equivalent to a certain family of variational states known as correlator-product-states (Clark, 2018, Glasser et al. 2018a). The question of determining how large are the respective classes of quantum states belonging to the NQS form, Eq. (3) and to computationally efficient tensor network is, however, still open. Exact representations of several intriguing phases of matter, including topological states and stabilizer codes (Deng et al. $2017 \mathrm{a}$; Glasser et al., 2018a; Huang and Moore, 2017; Kaubruegger et al., 2018, Lu et al., 2018; Zheng et al., 2018), have also been obtained in closed RBM form. Not surprisingly, given its shallow depth, RBM architectures are also expected to have limitations, on general grounds. Specifically, it is not in general possible to write all possible physical states in terms of compact RBM states (Gao and Duan, 2017). In order to lift the intrinsic limitations of RBMs, and efficiently describe a very large family of physical states, it is necessary to introduce deep Boltzmann Machines (DBM) with two hidden layers (Gao and Duan, 2017). Similar network constructions have been introduced also as a possible theoretical framework, alternative to the standard path-integral representation of quantum mechanics (Carleo et al. 2018).

\section{Learning from data}

Parallel to the activity on understanding the theoretical properties of NQS, a family of studies in this field is concerned with the problem of understanding how hard it is, in practice, to learn a quantum state from numerical data. This can be realized using either synthetic data (for example coming from numerical simulations) or directly from experiments.

This line of research has been explored in the supervised learning setting, to understand how well NQS can represent states that are not easily expressed (in closed analytic form) as ANN. The goal is then to train a NQS network $|\Psi\rangle$ to represent, as close as possible, a certain target state $|\Phi\rangle$ whose amplitudes can be efficiently computed. This approach has been successfully used to learn ground-states of fermionic, frustrated, and bosonic Hamiltonians (Cai and Liu, 2018). Those represent interesting study cases, since the sign/phase structure of the target wave-functions can pose a challenge to standard activation functions used in FFNN. Along the same lines, supervised approaches have been proposed to learn random matrix product states wave-functions both with shallow NQS (Borin and Abanin, 2019), and with generalized NQS including a computationally treatable DBM form (Pastori et al. 2018). While in the latter case these studies have revealed efficient strategies to perform the learning, in the former case hardness in learning some 
random MPS has been showed. At present, it is speculated that this hardness originates from the entanglement structure of the random MPS, however it is unclear if this is related to the hardness of the NQS optimization landscape or to an intrinsic limitation of shallow NQS.

Besides supervised learning of given quantum states, data-driven approaches with NQS have largely concentrated on unsupervised approaches. In this framework, only measurements from some target state $|\Phi\rangle$ or density matrix are available, and the goal is to reconstruct the full state, in NQS form, using such measurements. In the simplest setting, one is given a data set of $M$ measurements $\mathbf{r}^{(1)} \ldots \mathbf{r}^{(M)}$ distributed according to Born's rule prescription $P(\mathbf{r})=|\Phi(\mathbf{r})|^{2}$, where $P(\mathbf{r})$ is to be reconstructed. In cases when the wave-function is positive definite, or when only measurements in a certain basis are provided, reconstructing $P(\mathbf{r})$ with standard unsupervised learning approaches is enough to reconstruct all the available information on the underlying quantum state $\Phi$. This approach for example has been demonstrated for ground-states of stoquastic Hamiltonians (Torlai et al. 2018) using RBM-based generative models. An approach based on deep VAE generative models has also been demonstrated in the case of a family of classically-hard to sample from quantum states (Rocchetto et al. 2018), for which the effect of network depth has been shown to be beneficial for compression.

In the more general setting, the problem is to reconstruct a general quantum state, either pure or mixed, using measurements from more than a single basis of quantum numbers. Those are especially necessary to reconstruct also the complex phases of the quantum state. This problem corresponds to a well-known problem in quantum information, known as quantum state tomography, for which specific NQS approaches have been introduced (Carrasquilla et al., 2019, Torlai et al. 2018, Torlai and Melko, 2018). Those are discussed more in detail, in the dedicated section V.A, also in connection with other ML techniques used for this task.

\section{Variational Learning}

Finally, one of the main applications for the NQS representations is in the context of variational approximations for many-body quantum problems. The goal of these approaches is, for example, to approximately solve the Schrödinger equation using a NQS representation for the wave-function. In this case, the problem of finding the ground state of a given quantum Hamiltonian $H$ is formulated in variational terms as the problem of learning NQS weights $W$ minimizing $E(W)=$ $\langle\Psi(W)|H| \Psi(W)\rangle /\langle\Psi(W) \mid \Psi(W)\rangle$. This is achieved using a learning scheme based on variational Monte Carlo optimization (Carleo and Troyer, 2017). Within this family of applications, no external data representative of the quan- tum state is given, thus they typically demand a larger computational burden than supervised and unsupervised learning schemes for NQS.

Experiments on a variety of spin (Choo et al. 2018; Deng et al., 2017a; Glasser et al., 2018a; Liang et al. 2018), bosonic (Choo et al. | 2018; Saito, 2017, 2018; Saito and Kato, 2017), and fermionic (Han et al. 2018a; Luo and Clark, 2018; Nomura et al. , 2017) models have shown that results competitive with existing state-of-the-art approaches can be obtained. In some cases, improvement over existing variational results have been demonstrated, most notably for two-dimensional lattice models (Carleo and Troyer, 2017, Luo and Clark, 2018, Nomura et al. 2017) and for topological phases of matter (Glasser et al. 2018a; Kaubruegger et al., 2018).

Other NQS applications concern the solution of the time-dependent Schrödinger equation (Carleo and Troyer, 2017; Czischek et al., 2018, Fabiani and Mentink, 2019: Schmitt and Heyl, 2018). In these applications, one uses the time-dependent variational principle of Dirac and Frenkel (Dirac, 1930, Frenkel, 1934) to learn the optimal time evolution of network weights. This can be suitably generalized also to open dissipative quantum systems, for which a variational solution of the Lindblad equation can be realized (Hartmann and Carleo, 2019, Nagy and Savona, 2019; Vicentini et al., 2019; Yoshioka and Hamazaki, 2019).

In the great majority of the variational applications discussed here, the learning schemes used are typically higher-order techniques than standard SGD approaches. The stochastic reconfiguration (SR) approach (Becca and Sorella, 2017; Sorella, 1998) and its generalization to the time-dependent case (Carleo et al., 2012), have proven particularly suitable to variational learning of NQS. The SR scheme can be seen as a quantum analogous of the natural-gradient method for learning probability distributions (Amari 1998), and builds on the intrinsic geometry associated with the neural-network parameters. More recently, in an effort to use deeper and more expressive networks than those initially adopted, learning schemes building on first-order techniques have been more consistently used (Kochkov and Clark, 2018, Sharir et al., 2019). These constitute two different philosophy of approaching the same problem. On one hand, early applications focused on small networks learned with very accurate but expensive training techniques. On the other hand, later approaches have focused on deeper networks and cheaper - but also less accurate- learning techniques. Combining the two philosophy in a computationally efficient way is one of the open challenges in the field.

\section{B. Speed up many-body simulations}

The use of ML methods in the realm of the quantum many-body problems extends well beyond neural- 
network representation of quantum states. A powerful technique to study interacting models are Quantum Monte Carlo (QMC) approaches. These methods stochastically compute properties of quantum systems through mapping to an effective classical model, for example by means of the path-integral representation. A practical issue often resulting from these mappings is that providing efficient sampling schemes of high-dimensional spaces (path integrals, perturbation series, etc..) requires a careful tuning, often problem-dependent. Devising general-purpose samplers for these representations is therefore a particularly challenging problem. Unsupervised ML methods can, however, be adopted as a tool to speed-up Monte Carlo sampling for both classical and quantum applications. Several approaches in this direction have been proposed, and leverage the ability of unsupervised learning to well approximate the target distribution being sampled from in the underlying Monte Carlo scheme. Relatively simple energy-based generative models have been used in early applications for classical systems (Huang and Wang, 2017; Liu et al., 2017b). "Self-learning" Monte Carlo techniques have then been generalized also to fermionic systems (Chen et al. 2018a, Liu et al., 2017c, Nagai et al., 2017). Overall, it has been found that such approaches are effective at reducing the autocorrelation times, especially when compared to families of less effective Markov Chain Monte Carlo with local updates. More recently, state-of-the-art generative ML models have been adopted to speed-up sampling in specific tasks. Notably, (Wu et al. 2018) have used deep autoregressive models that may enable a more efficient sampling from hard classical problems, such as spin glasses. The problem of finding efficient sampling schemes for the underlying classical models is then transformed into the problem of finding an efficient corresponding autoregressive deep network representation. This approach has also been generalized to the quantum cases in Sharir et al. 2019), where an autoregressive representation of the wave-function is introduced. This representation is automatically normalized and allows to bypass Markov Chain Monte Carlo in the variational learning discussed above.

While exact for a large family of bosonic and spin systems, QMC techniques typically incur in a severe sign problem when dealing with several interesting fermionic models, as well as frustrated spin Hamiltonians. In this case, it is tempting to use ML approaches to attempt a direct or indirect reduction of the sign problem. While only in its first stages, this family of applications has been used to infer information about fermionic phases through hidden information in the Green's function (Broecker et al. 2017b).

Similarly, ML techniques can help reduce the burden of more subtle manifestations of the sign problem in dynamical properties of quantum models. In particular, the problem of reconstructing spectral functions from imaginary-time correlations in imaginary time is also a field in which ML can be used as an alternative to traditional maximum-entropy techniques to perform analytical continuations of QMC data (Arsenault et al., 2017; Fournier et al., 2018, Yoon et al., 2018).

\section{Classifying many-body quantum phases}

The challenge posed by the complexity of many-body quantum states manifests itself in many other forms. Specifically, several elusive phases of quantum matter are often hard to characterize and pinpoint both in numerical simulations and in experiments. For this reason, ML schemes to identify phases of matter have become particularly popular in the context of quantum phases. In the following we review some of the specific applications to the quantum domain, while a more general discussion on identifying phases and phase transitions is to be found in II.E.

\section{Synthetic data}

Following the early developments in phase classifications with supervised approaches (Carrasquilla and Melko, 2017, Van Nieuwenburg et al. 2017, Wang, 2016), many studies have since then focused on analyzing phases of matter in synthetic data, mostly from simulations of quantum systems. While we do not attempt here to provide an exhaustive review of the many studies appeared in this direction, we highlight two large families of problems that have so-far largely served as benchmarks for new ML tools in the field.

A first challenging test bench for phase classification schemes is the case of quantum many-body localization. This is an elusive phase of matter showing characteristic fingerprints in the many-body wave-function itself, but not necessarily emerging from more traditional order parameters [see for example Alet and Laflorencie, 2018) for a recent review on the topic]. First studies in this direction have focused on training strategies aiming at the Hamiltonian or entanglement spectra $(\mathrm{Hsu}$ et al. 2018; Huembeli et al., 2018b; Schindler et al., 2017; Venderley et al., 2018, Zhang et al., 2019). These works have demonstrated the ability to very effectively learn the MBL phase transition in relatively small systems accessible with exact diagonalization techniques. Other studies have instead focused on identifying signatures directly in experimentally relevant quantities, most notably from the many-body dynamics of local quantities (Doggen et al., 2018, van Nieuwenburg et al., 2018). The latter schemes appear to be at present the most promising for applications to experiments, while the former have been used as a tool to identify the existence of an unexpected phase in the presence of correlated disorder $(\mathrm{Hsu}$ 
et al. 2018).

Another very challenging class of problems is found when analyzing topological phases of matter. These are largely considered a non-trivial test for ML schemes, because these phases are typically characterized by nonlocal order parameters. In turn, these non-local order parameters are hard to learn for popular classification schemes used for images. This specific issue is already present when analyzing classical models featuring topological phase transitions. For example, in the presence of a BKT-type transition, learning schemes trained on raw Monte Carlo configurations are not effective (Beach et al. 2018. Hu et al., 2017). These problems can be circumvented devising training strategies using pre-engineered features (Broecker et al. 2017a; Cristoforetti et al., 2017; Wang and Zhai,|2017; Wetzel|,2017) instead of raw Monte Carlo samples. These features typically rely on some important a-priori assumptions on the nature of the phase transition to be looked for, thus diminishing their effectiveness when looking for new phases of matter. Deeper in the quantum world, there has been research activity along the direction of learning, in a supervised fashion, topological invariants. Neural networks can be used for example to classify families of non-interacting topological Hamiltonians, using as an input their discretized coefficients, either in real (Ohtsuki and Ohtsuki, 2016, 2017) or momentum space (Sun et al. | 2018, Zhang et al. | 2018c). In these cases, it is found that neural networks are able to reproduce the (already known beforehand) topological invariants, such as winding numbers, Berry curvatures and more. The context of strongly-correlated topological matter is, to a large extent, more challenging than the case of non-interacting band models. In this case, a common approach is to define a set of carefully preengineered features to be used on top of the raw data. One well known example is the case of of the so-called quantum loop topography (Zhang and Kim, 2017), trained on local operators computed on single shots of sampled wave-function walkers, as for example done in variational Monte Carlo. It has been shown that this very specific choice of local features is able to distinguish strongly interacting fraction Chern insulators, and also $\mathbb{Z}_{2}$ quantum spin liquids (Zhang et al., 2017). Similar efforts have been realized to classify more exotic phases of matter, including magnetic skyrmion phases (Iakovlev et al. 2018), and dynamical states in antiskyrmion dynamics (Ritzmann et al., 2018).

Despite the progress seen so far along the many direction described here, it is fair to say that topological phases of matter, especially for interacting systems, constitute one of the main challenges for phase classification. While some good progress has already been made (Huembeli et al. 2018a; Rodriguez-Nieva and Scheurer, 2018), future research will need to address the issue of finding training schemes not relying on pre-selection of data features.

\section{Experimental data}

Beyond extensive studies on data from numerical simulations, supervised schemes have found their way also as a tool to analyze experimental data from quantum systems. In ultra-cold atoms experiments, supervised learning tools have been used to map out both the topological phases of non-interacting particles, as well the onset of Mott insulating phases in finite optical traps (Rem et al. 2018). In this specific case, the phases where already known and identifiable with other approaches. However, ML-based techniques combining a-priori theoretical knowledge with experimental data hold the potential for genuine scientific discovery.

For example, ML can enable scientific discovery in the interesting cases when experimental data has to be attributed to one of many available and equally likely apriory theoretical models, but the experimental information at hand is not easily interpreted. Typically interesting cases emerge for example when the order parameter is a complex, and only implicitly known, non-linear function of the experimental outcomes. In this situation, ML approaches can be used as a powerful tool to effectively learn the underlying traits of a given theory, and provide a possibly unbiased classification of experimental data. This is the case for incommensurate phases in high-temperature superconductors, for which scanning tunneling microscopy images reveal complex patters that are hard to decipher using conventional analysis tools. Using supervised approaches in this context, recent work (Zhang et al. 2018d) has shown that is possible to infer the nature of spatial ordering in these systems, also see Fig. 5 .

A similar idea has been also used for another prototypical interacting quantum systems of fermions, the Hubbard model, as implemented in ultra-cold atoms experiments in optical lattices. In this case the reference models provide snapshots of the thermal density matrix that can be pre-classified in a supervised learning fashion. The outcome of this study (Bohrdt et al., 2018), is that the experimental results are with good confidence compatible with one of the theories proposed, in this case a geometric string theory for charge carriers.

In the last two experimental applications described above, the outcome of the supervised approaches are to a large extent highly non-trivial, and hard to predict a priori on the basis of other information at hand. The inner bias induced by the choice of the theories to be classified is however one of the current limitations that these kind of approaches face.

\section{Tensor networks for machine learning}

The research topics reviewed so far are mainly concerned with the use of ML ideas and tools to study prob- 


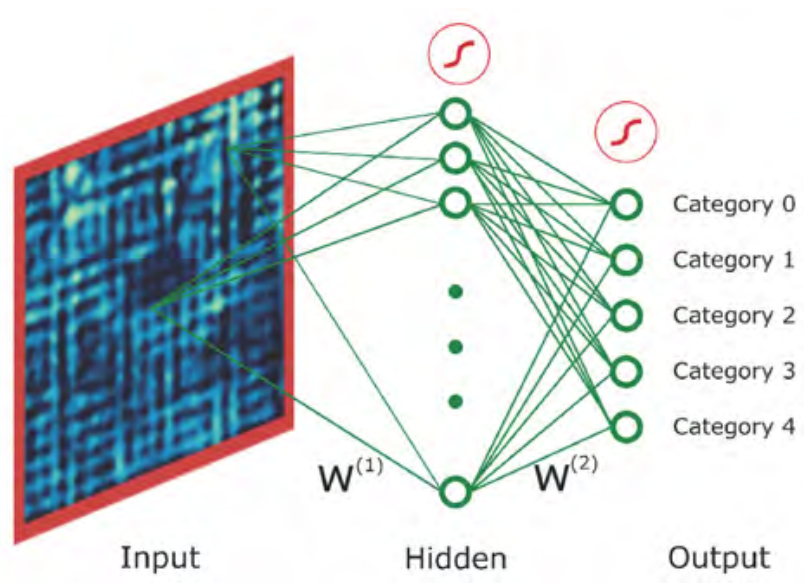

Figure 5 Example of machine learning approach to the classification of experimental images from scanning tunneling microscopy of high-temperature superconductors. Images are classified according to the predictions of distinct types of periodic spatial modulations. Reproduced from (Zhang et al. 2018d)

lems in the realm of quantum many-body physics. Complementary to this philosophy, an interesting research direction in the field explores the inverse direction, investigating how ideas from quantum many-body physics can inspire and devise new powerful ML tools. Central to these developments are tensor-network representations of many-body quantum states. These are very successful variational families of many-body wave functions, naturally emerging from low-entanglement representations of quantum states (Verstraete et al. 2008). Tensor networks can serve both as a practical and a conceptual tool for ML tasks, both in the supervised and in the unsupervised setting.

These approaches build on the idea of providing physics-inspired learning schemes and network structures alternative to the more conventionally adopted stochastic learning schemes and FFNN networks. For example, matrix product state (MPS) representations, a work-horse for the simulation of interacting one-dimensional quantum systems (White, 1992), have been re-purposed to perform classification tasks, (Liu et al. , 2018, Novikov et al., 2016, Stoudenmire and Schwab, 2016), and also recently adopted as explicit generative models for unsupervised learning (Han et al., 2018b, Stokes and Terilla, 2019). It is worth mentioning that other related high-order tensor decompositions, developed in the context of applied mathematics have been used for ML purposes (Acar and Yener, 2009, Anandkumar et al. 2014). Tensor-train decompositions (Oseledets, 2011), formally equivalent to MPS representations, have been introduced in parallel as a tool to perform various machine learning tasks (Gorodetsky et al., 2019, Izmailov et al., 2017, Novikov et al., 2016). Networks closely related to MPS have also been explored for time-series modeling (Guo et al. 2018).

In the effort of increasing the amount of entanglement encoded in these low-rank tensor decompositions, recent works have concentrated on tensor-network representations alternative to the MPS form. One notable example is the use of tree tensor networks with a hierarchical structure (Hackbusch and Kühn, 2009, Shi et al. 2006), which have been applied to classification (Liu et al., 2017a, Stoudenmire, 2018) and generative modeling (Cheng et al., 2019) tasks with good success. Another example is the use of entangled plaquette states (Changlani et al. 2009, Gendiar and Nishino, 2002, Mezzacapo et al. 2009) and string bond states (Schuch et al., 2008), both showing sizable improvements in classification tasks over MPS states (Glasser et al., 2018b).

On the more theoretical side, the deep connection between tensor networks and complexity measures of quantum many-body wave-functions, such as entanglement entropy, can be used to understand, and possible inspire, successful network designs for ML purposes. The tensornetwork formalism has proven powerful in interpreting deep learning through the lens of renormalization group concepts. Pioneering work in this direction has connected MERA tensor network states (Vidal, 2007) to hierarchical Bayesian networks (Bény, 2013). In later analysis, convolutional arithmetic circuits (Cohen et al., 2016), a family of convolutional networks with product nonlinearities, have been introduced as a convenient model to bridge tensor decompositions with FFNN architectures. Beside their conceptual relevance, these connections can help clarify the role of inductive bias in modern and commonly adopted neural networks (Levine et al., 2017).

\section{E. Outlook and Challenges}

Applications of ML to quantum many-body problems have seen a fast-pace progress in the past few years, touching a diverse selection of topics ranging from numerical simulation to data analysis. The potential of ML techniques has already surfaced in this context, already showing improved performance with respect to existing techniques on selected problems. To a large extent, however, the real power of ML techniques in this domain has been only partially demonstrated, and several open problems remain to be addressed.

In the context of variational studies with NQS, for example, the origin of the empirical success obtained so far with different kind of neural network quantum states is not equally well understood as for other families of variational states, like tensor networks. Key open challenges remain also with the representation and simulation of fermionic systems, for which efficient neural-network representation are still to be found.

Tensor-network representations for ML purposes, as 
well as complex-valued networks like those used for NQS, play an important role to bridge the field back to the arena of computer science. Challenges for the future of this research direction consist in effectively interfacing with the computer-science community, while retaining the interests and the generality of the physics tools.

For what concerns ML approaches to experimental data, the field is largely still in its infancy, with only a few applications having been demonstrated so far. This is in stark contrast with other fields, such as High-Energy and Astrophysics, in which ML approaches have matured to a stage where they are often used as standard tools for data analysis. Moving towards achieving the same goal in the quantum domain demands closer collaborations between the theoretical and experimental efforts, as well as a deeper understanding of the specific problems where ML can make a substantial difference.

Overall, given the relatively short time span in which applications of ML approaches to many-body quantum matter have emerged, there are however good reasons to believe that these challenges will be energetically addressed-and some of them solved-in the coming years.

\section{QUANTUM COMPUTING}

Quantum computing uses quantum systems to process information. In the most popular framework of gatebased quantum computing (Nielsen and Chuang, 2002), a quantum algorithm describes the evolution of an initial state $\left|\psi_{0}\right\rangle$ of a quantum system of $n$ two-level systems called qubits to a final state $\left|\psi_{f}\right\rangle$ through discrete transformations or quantum gates. The gates usually act only on a small number of qubits, and the sequence of gates defines the computation.

The intersection of machine learning and quantum computing has become an active research area in the last couple of years, and contains a variety of ways to merge the two disciplines (see also (Dunjko and Briegel, 2018) for a review). Quantum machine learning asks how quantum computers can enhance, speed up or innovate machine learning (Biamonte et al., 2017; Ciliberto et al. 2018, Schuld and Petruccione, 2018a) (see also Sections VII and (V). Quantum learning theory highlights theoretical aspects of learning under a quantum framework (Arunachalam and de Wolf, 2017).

In this Section we are concerned with a third angle, namely how machine learning can help us to build and study quantum computers. This angle includes topics ranging from the use of intelligent data mining methods to find physical regimes in materials that can be used as qubits (Kalantre et al. 2019), to the verification of quantum devices (Agresti et al.||2019), learning the design of quantum algorithms (Bang et al., 2014, Wecker et al. 2016), facilitating classical simulations of quantum circuits (Jónsson et al. 2018), automated design on quan- tum experiments Krenn et al. 2016, Melnikov et al. 2018), and learning to extract relevant information from measurements (Seif et al. 2018).

We focus on three general problems related to quantum computing which were targeted by a range of ML methods: the problem of reconstructing en benchmarking quantum states via measurements; the problem of preparing a quantum state via quantum control; the problem of maintaining the information stored in the state through quantum error correction. The first problem is known as quantum state tomography, and it is especially useful to understand and improve upon the limitations of current quantum hardware. Quantum control and quantum error corrections solve related problems, however usually the former refers to hardware-related solutions while the latter uses algorithmic solutions to the problem of executing a computational protocol with a quantum system.

Similar to the other disciplines in this review, machine learning has shown promising results in all these areas, and will in the longer run likely enter the toolbox of quantum computing to be used side-by-side with other well-established methods.

\section{A. Quantum state tomography}

The general goal of quantum state tomography (QST) is to reconstruct the density matrix of an unknown quantum state, through experimentally available measurements. QST is a central tool in several fields of quantum information and quantum technologies in general, where it is often used as a way to assess the quality and the limitations of the experimental platforms. The resources needed to perform full QST are however extremely demanding, and the number of required measurements scales exponentially with the number of qubits/quantum degrees of freedom [see (Paris and Rehacek, 2004) for a review on the topic, and (Haah et al., 2017, O'Donnell and Wright, 2016) for a discussion on the hardness of learning in state tomography].

ML tools have been identified already several years ago as a tool to improve upon the cost of full QST, exploiting some special structure in the density matrix. Compressed sensing (Gross et al. 2010) is one prominent approach to the problem, allowing to reduce the number of required measurements from $d^{2}$ to $\mathcal{O}\left(r d \log (d)^{2}\right)$, for a density matrix of rank $r$ and dimension $d$. Successful experimental realization of this technique has been for example implemented for a six-photon state (Tóth et al. 2010) or a seven-qubit system of trapped ions (Riofrío et al., 2017). On the methodology side, full QST has more recently seen the development of deep learning approaches. For example, using a supervised approach based on neural networks having as an output the full density matrix, and as an input possible measurement 
outcomes (Xu and $\mathrm{Xu}, 2018)$. The problem of choosing optimal measurement basis for QST has also been recently addressed using a neural-network based approach that optimizes the prior distribution on the target density matrix, using Bayes rule (Quek et al., 2018 ). In general, while ML approaches to full QST can serve as a viable tool to alleviate the measurement requirements, they cannot however provide an improvement over the intrinsic exponential scaling of QST.

The exponential barrier can be typically overcome only in situations when the quantum state is assumed to have some specific regularity properties. Tomography based on tensor-network paremeterizations of the density matrix has been an important first step in this direction, allowing for tomography of large, low-entangled quantum systems (Lanyon et al. , 2017). ML approaches to parameterization-based QST have emerged in recent times as a viable alternative, especially for highly entangled states. Specifically, assuming a NQS form (see Eq. 3 in the case of pure states) QST can be reformulated as an unsupervised ML learning task. A scheme to retrieve the phase of the wave-function, in the case of pure states, has been demonstrated in (Torlai et al. 2018). In these applications, the complex phase of the many-body wave-function is retrieved upon reconstruction of several probability densities associated to the measurement process in different basis. Overall, this approach has allowed to demonstrate QST of highly entangled states up to about 100 qubits, unfeasible for full QST techniques. This tomography approach can be suitably generalized to the case of mixed states introducing parameterizations of the density matrix based either on purified NQS (Torlai and Melko, 2018) or on deep normalizing flows (Cranmer et al. 2 2019). The former approach has been also demonstrated experimentally with Rydberg atoms (Torlai et al. 2019). An interesting alternative to the NQS representation for tomographic purposes has also been recently suggested (Carrasquilla et al. 2019 ). This is based on parameterizing the density matrix directly in terms of positive-operator valued measure (POVM) operators. This approach therefore has the important advantage of directly learning the measurement process itself, and has been demonstrated to scale well on rather large mixed states. A possible inconvenient of this approach is that the density matrix is only implicitly defined in terms of generative models, as opposed to explicit parameterizations found in NQS-based approaches.

Other approaches to QST have explored the use of quantum states parameterized as ground-states of local Hamiltonians (Xin et al., 2018), or the intriguing possibility of bypassing QST to directly measure quantum entanglement (Gray et al., 2018). Extensions to the more complex problem of quantum process tomography are also promising (Banchi et al. 2018$)$, while the scalability of ML-based approaches to larger systems still presents challenges.
Finally, the problem of learning quantum states from experimental measurements has also profound implications on the understanding of the complexity of quantum systems. In this framework, the PAC learnability of quantum states (Aaronson, 2007), experimentally demonstrated in (Rocchetto et al., 2017), and the "shadow tomography" approach (Aaronson, 2017), showed that even linearly sized training sets can provide sufficient information to succeed in certain quantum learning tasks. These information-theoretic guarantees come with computational restrictions and learning is efficient only for special classes of states (Rocchetto, 2018)

\section{B. Controlling and preparing qubits}

A central task of quantum control is the following: Given an evolution $U(\theta)$ that depends on parameters $\theta$ and maps an initial quantum state $\left|\psi_{0}\right\rangle$ to $|\psi(\theta)\rangle=$ $U(\theta)\left|\psi_{0}\right\rangle$, which parameters $\theta^{*}$ minimise the overlap or distance between the prepared state and the target state, $\left|\left\langle\psi(\theta) \mid \psi_{\text {target }}\right\rangle\right|^{2}$ ? To facilitate analytic studies, the space of possible control interventions is often discretized, so that $U(\theta)=U\left(s_{1}, \ldots, s_{T}\right)$ becomes a sequence of steps $s_{1}, \ldots, s_{T}$. For example, a control field could be applied at only two different strengths $h_{1}$ and $h_{2}$, and the goal is to find an optimal strategy $s_{t} \in\left\{h_{1}, h_{2}\right\}, t=1, \ldots, T$ to bring the initial state as close as possible to the target state using only these discrete actions.

This setup directly generalizes to a reinforcement learning framework (Sutton and Barto, 2018), where an agent picks "moves" from the list of allowed control interventions, such as the two field strengths applied to the quantum state of a qubit. This framework has proven to be competitive to state-of-the-art methods in various settings, such as state preparation in non-integrable manybody quantum systems of interacting qubits (Bukov et al. 2018), or the use of strong periodic oscillations to prepare so-called "Floquet-engineered" states (Bukov, 2018). A recent study comparing (deep) reinforcement learning with traditional optimization methods such as Stochastic Gradient Descent for the preparation of a single qubit state shows that learning is of advantage if the "action space" is naturally discretized and sufficiently small (Zhang et al., 2019).

The picture becomes increasingly complex in slightly more realistic settings, for example when the control is noisy (Niu et al. 2018). In an interesting twist, the control problem has also been tackled by predicting future noise using a recurrent neural network that analyses the time series of past noise. Using the prediction, the anticipated future noise can be corrected (Mavadia et al. 2017).

An altogether different approach to state preparation with machine learning tries to find optimal strategies for evaporative cooling to create Bose-Einstein condensates 
(Wigley et al. 2016). In this online optimization strategy based on Bayesian optimization (Frazier, 2018; Jones et al., 1998), a Gaussian process is used as a statistical model that captures the relationship between the control parameters and the quality of the condensate. The strategy discovered by the machine learning model allows for a cooling protocol that uses 10 times fewer iterations than pure optimization techniques. An interesting feature is that - contrary to the common reputation of machine learning - the Gaussian process allows to determine which control parameters are more important than others.

Another angle is captured by approaches that 'learn' the sequence of optical instruments in order to prepare highly entangled photonic quantum states (Melnikov et al. 2018).

\section{Error correction}

One of the major challenges in building a universal quantum computer is error correction. During any computation, errors are introduced by physical imperfections of the hardware. But while classical computers allow for simple error correction based on duplicating information, the no-cloning theorem of quantum mechanics requires more complex solutions. The most well-known proposal of surface codes prescribes to encode one "logical qubit" into a topological state of several "physical qubits". Measurements on these physical qubits reveal a "footprint" of the chain of error events called a syndrome. A decoder maps a syndrome to an error sequence, which, once known, can be corrected by applying the same error sequence again, and without affecting the logical qubits that store the actual quantum information. Roughly stated, the art of quantum error correction is therefore to predict errors from a syndrome - a task that naturally fits the framework of machine learning.

In the past few years, various models have been applied to quantum error correction, ranging from supervised to unsupervised and reinforcement learning. The details of their application became increasingly complex. One of the first proposals deploys a Boltzmann machine trained by a data set of pairs (error, syndrome), which specifies the probability $p$ (error, syndrome), which can be used to draw samples from the desired distribution $p$ (error|syndrome) (Torlai and Melko, 2017). This simple recipe shows a performance for certain kinds of errors comparable to common benchmarks. The relation between syndromes and errors can likewise be learned by a feed-forward neural network (Krastanov and Jiang, 2017 Maskara et al., 2019; Varsamopoulos et al., 2017). However, these strategies suffer from scalability issues, as the space of possible decoders explodes and data acquisition becomes an issue. More recently, neural networks have been combined with the concept of renormalization group to address this problem (Varsamopoulos et al., 2018), and the significance of different hyper-parameters of the neural network has been studied (Varsamopoulos et al. 2019).

Besides scalability, an important problem in quantum error correction is that the syndrome measurement procedure could also introduce an error, since it involves applying a small quantum circuit. This setting increases the problem complexity but is essential for real applications. Noise in the identification of errors can be mitigated by doing repeated cycles of syndrome measurements. To consider the additional time dimension, recurrent neural network architectures have been proposed (Baireuther et al. 2018). Another avenue is to consider decoding as a reinforcement learning problem (Sweke et al., 2018), in which an agent can choose consecutive operations acting on physical qubits (as opposed to logical qubits) to correct for a syndrome and gets rewarded if the sequence corrected the error.

While much of machine learning for error correction focuses on surface codes that represent a logical qubit by physical qubits according to some set scheme, reinforcement agents can also be set up agnostic of the code (one could say they learn the code along with the decoding strategy). This has been done for quantum memories, a system in which quantum states are supposed to be stored rather than manipulated (Nautrup et al., 2018), as well as in a feedback control framework which protects qubits against decoherence (Fösel et al. 2018). Finally, beyond traditional reinforcement learning, novel strategies such as projective simulation can be used to combat noise (Tiersch et al., 2015).

As a summary, machine learning for quantum error correction is a problem with several layers of complexity that, for realistic applications, requires rather complex learning frameworks. Nevertheless, it is a very natural candidate for machine learning, and especially reinforcement learning.

\section{CHEMISTRY AND MATERIALS}

Machine learning approaches have been applied to predict the energies and properties of molecules and solids, with the popularity of such applications increasing dramatically. The quantum nature of atomic interactions makes energy evaluations computationally expensive, so ML methods are particularly useful when many such calculations are required. In recent years, the everexpanding applications of ML in chemistry and materials research include predicting the structures of related molecules, calculating energy surfaces based on molecular dynamics (MD) simulations, identifying structures that have desired material properties, and creating machinelearned density functionals. For these types of problems, input descriptors must account for differences in atomic 
environments in a compact way. Much of the current work using ML for atomistic modeling is based on early work describing the local atomic environment with symmetry functions for input into a atom-wise neural network (Behler and Parrinello, 2007), representing atomic potentials using Gaussian process regression methods (Bartók et al. 2010), or using sorted interatomic distances weighted by the nuclear charge (the "Coulomb matrix") as a molecular descriptor (Rupp et al., 2012). Continuing development of suitable structural representations is reviewed by Behler (2016). A discussion of ML for chemical systems in general, including learning structure-property relationships, is found in the review by Butler et al. (2018), with additional focus on dataenabled theoretical chemistry reviewed by Rupp et al. (2018). In the sections below, we present recent examples of ML applications in chemical physics.

\section{A. Energies and forces based on atomic environments}

One of the primary uses of ML in chemistry and materials research is to predict the relative energies for a series of related systems, most typically to compare different structures of the same atomic composition. These applications aim to determine the structure(s) most likely to be observed experimentally or to identify molecules that may be synthesizable as drug candidates. As examples of supervised learning, these ML methods employ various quantum chemistry calculations to label molecular representations $(X \mu)$ with corresponding energies $(y \mu)$ to generate the training (and test) data sets $\left\{X_{\mu}, y_{\mu}\right\}_{\mu=1}^{n}$. For quantum chemistry applications, NN methods have had great success in predicting the relative energies of a wide range of systems, including constitutional isomers and non-equilibrium configurations of molecules, by using many-body symmetry functions that describe the local atomic neighborhood of each atom (Behler, 2016). Many successes in this area have been derived from this type of atom-wise decomposition of the molecular energy, with each element represented using a separate NN (Behler and Parrinello, 2007) (see Fig. 6(a)). For example, ANI1 is a deep NN potential successfully trained to return the density functional theory (DFT) energies of any molecule with up to 8 heavy atoms ( $\mathrm{H}, \mathrm{C}, \mathrm{N}, \mathrm{O}$ ) (Smith et al. 2017). In this work, atomic coordinates for the training set were selected using normal mode sampling to include some vibrational perturbations along with optimized geometries. Another example of a general NN for molecular and atomic systems is the Deep Potential Molecular Dynamics (DPMD) method specifically created to run MD simulations after being trained on energies from bulk simulations (Zhang et al.,2018a). Rather than simply include non-local interactions via the total energy of a system, another approach was inspired by the manybody expansion used in standard computational physics.
In this case adding layers to allow interactions between atom-centered NNs improved the molecular energy predictions (Lubbers et al. 2018).

The examples above use translation- and rotationinvariant representations of the atomic environments, thanks to the incorporation of symmetry functions in the NN input. For some applications, such as describing molecular reactions and materials phase transformations, atomic representations must also be continuous and differentiable. The smooth overlap of atomic positions (SOAP) kernels address all of these requirements by including a similarity metric between atomic environments (Bartók et al., 2013). Recent work to preserve symmetries in alternate molecular representations addresses this problem in different ways. To capitalize on known molecular symmetries for "Coulomb matrix" inputs, both bonding (rigid) and dynamic symmetries have been incorporated to improve the coverage of training data in the configurational space (Chmiela et al. 2018). This work also includes forces in the training, allowing for MD simulations at the level of coupled cluster calculations for small molecules, which would traditionally be intractable. Molecular symmetries can also be learned, as shown in determining local environment descriptors that make use of continuous-filter convolutions to describe atomic interactions (Schütt et al. 2018). Further development of atom environment descriptors that are compact, unique, and differentiable will certainly facilitate new uses for ML models in the study of molecules and materials.

However, machine learning has also been applied in ways that are more closely integrated with conventional approaches, so as to be more easily incorporated in existing codes. For example, atomic charge assignments compatible with classical force fields can be learned, without the need to run a new quantum mechanical calculation for each new molecule of interest (Sifain et al. 2018 ). In addition, condensed phase simulations for molecular species require accurate intra- and intermolecular potentials, which can be difficult to parameterize. To this end, local NN potentials can be combined with physicallymotivated long-range Coulomb and van der Waals contributions to describe larger molecular systems (Yao et al. 2018). Local ML descriptions can also be successfully combined with many-body expansion methods to allow application of ML potentials to larger systems, as demonstrated for water clusters (Nguyen et al. , 2018). Alternatively, intermolecular interactions can be fitted to a set of ML models trained on monomers to create a transferable model for dimers and clusters (Bereau et al., 2018$)$.

\section{B. Potential and free energy surfaces}

Machine learning methods are also employed to describe free energy surfaces. Rather than learning the po- 

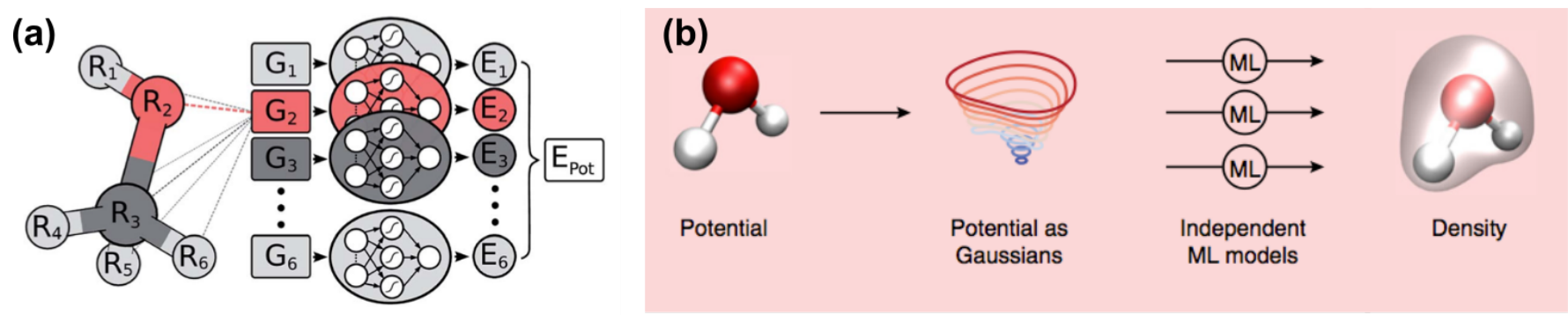

Figure 6 Several representations are currently used to describe molecular systems in ML models, including (a) atomic coordinates, with symmetry functions encoding local bonding environments, as inputs to element-based neural networks (Reproduced from (Gastegger et al. 2017)) and (b) nuclear potentials approximated by a sum of Gaussian functions as inputs kernel ridge regression models for electron densities (Modified from (Brockherde et al. 2017 )).

tential energy of each molecular conformation directly as described above, an alternate approach is to learn the free energy surface of a system as a function of collective variables, such as global Steinhardt order parameters or a local dihedral angle for a set of atoms. A compact ML representation of a free energy surface (FES) using a NN allows improved sampling of the high dimensional space when calculating observables that depend on an ensemble of conformers. For example, a learned FES can be sampled to predict the isothermal compressibility of solid xenon under pressure, or the expected NMR spinspin J couplings of a peptide (Schneider et al., 2017). Small NN's representing a FES can also be trained iteratively using data points generated by on-the-fly adaptive sampling (Sidky and Whitmer, 2018). This promising approach highlights the benefit of using a smooth representation of the full configurational space when using the ML models themselves to generate new training data. As the use of machine-learned FES representations increases, it will be important to determine the limit of accuracy for small NN's and how to use these models as a starting point for larger networks or other ML architectures.

Once the relevant minima have been identified on a FES, the next challenge is to understand the processes that take a system from one basin to another. For example, developing a Markov state model to describe conformational changes requires dimensionality reduction to translate molecular coordinates into the global reaction coordinate space. To this end, the power of deep learning with time-lagged autoencoder methods has been harnessed to identify slowly changing collective variables in peptide folding examples (Wehmeyer and Noé, 2018). A variational NN-based approach has also been used to identify important kinetic processes during protein folding simulations and provides a framework for unifying coordinate transformations and FES surface exploration (Mardt et al., 2018). A promising alternate approach is to use ML to sample conformational distributions directly. Boltzmann generators can sample the equilibrium distribution of a collective variable space and sub- sequently provide a set of states that represent the distribution of states on the FES (Noé et al., 2019).

Furthermore, the long history of finding relationships between minima on complex energy landscapes may also be useful as we learn to understand why ML models exhibit such general success. Relationships between the methods and ideas currently used to describe molecular systems and the corresponding are reviewed in Ballard et al. 2017). Going forward, the many tools developed by physicists to explore and quantify features on energy landscapes may be helpful in creating new algorithms to efficiently optimize model weights during training. (See also the related discussion in Sec. II.D.4) This area of interdisciplinary research promises to yield methods that will be useful in both machine learning and physics fields.

\section{Materials properties}

Using learned interatomic potentials based on local environments has also afforded improvement in the calculation of materials properties. Matching experimental data typically requires sampling from the ensemble of possible configurations, which comes at a considerable cost when using large simulation cells and conventional methods. Recently, the structure and material properties of amorphous silicon were predicted using molecular dynamics (MD) with a ML potential trained on density functional theory (DFT) calculations for only small simulation cells (Deringer et al. . 2018). Related applications of using ML potentials to model the phase change between crystalline and amorphous regions of materials such as GeTe and amorphous carbon are reviewed by Sosso et al. (2018). Generating a computationally-tractable potential that is sufficiently accurate to describe phase changes and the relative energies of defects on both an atomistic and material scale is quite difficult, however the recent success for silicon properties indicates that ML methods are up to the challenge (Bartók et al. 2018).

Ideally, experimental measurements could also be incorporated in data-driven ML methods that aim to predict material properties. However, reported results are 
too often limited to high-performance materials with no counter examples for the training process. In addition, noisy data is coupled with a lack of precise structural information needed for input into the ML model. For for organic molecular crystals, these challenges were overcome for predictions of NMR chemical shifts, which are very sensitive to local environments, by using a Gaussian process regression framework trained on DFT-calculated values of known structures (Paruzzo et al., 2018). Matching calculated values with experimental results prior to training the ML model enabled the validation of a predicted pharmaceutical crystal structure.

Other intriguing directions include identification of structurally similar materials via clustering and using convex hull construction to determine which of the many predicted structures should be most stable under certain thermodynamic constraints (Anelli et al. 2018). Using kernel-PCA descriptors for the construction of the convex hull has been applied to identify crystalline ice phases and was shown to cluster thousands structures which differ only by proton disorder or stacking faults (Engel et al. 2018) (see Fig. 7). Machine-learned methods based on a combination of supervised and unsupervised techniques certainly promises to be a fruitful research area in the future. In particular, it remains an exciting challenge to identify, predict, or even suggest materials that exhibit a particular desired property.

\section{Electron densities for density functional theory}

In many of the examples above, density functional theory calculations have been used as the source of training data. It is fitting that machine learning is also playing a role in creating new density functionals. Machine learning is a natural choice for situations such as DFT where we do not have knowledge of the functional form of an exact solution. The benefit of this approach to identifying a density functional was illustrated by approximating the kinetic energy functional of an electron distribution in a 1D potential well (Snyder et al., 2012). For use in standard Kohn-Sham based DFT codes, the derivative of the ML functional must also be used to find the appropriate ground state electron distribution. Using kernel ridge regression without further modification can lead to noisy derivatives, but projecting the resulting energies back onto the learned space using PCA resolves this issue (Li et al. 2015). A NN-based approach to learning the exchange-correlation potential has also been demonstrated for 1D systems (Nagai et al. 2018). In this case, the ML method makes direct use of the derivatives generated during the NN training steps.

It is also possible to bypass the functional derivative entirely by using ML to generate the appropriate ground state electron density that corresponds to a nuclear potential (Brockherde et al. 2017), as shown in Fig. 6(b).
Furthermore, this work demonstrated that the energy of a molecular system can also be learned with electron densities as an input, enabling reactive MD simulations of proton transfer events based on DFT energies. Intriguingly, an approximate electron density, such as a sum of densities from isolated atoms, has also been successfully employed as the input for predicting molecular energies (Eickenberg et al. 2018). A related approach for periodic crystalline solids used local electron densities from an embedded atom method to train Bayesian ML models to return total system energies (Schmidt et al. 2018). Since the total energy is an extensive property, a scalable NN model based on summation of local electron densities has also been developed to run large DFT-based simulations for 2D porous graphene sheets (Mills et al. 2019). With these successes, it has become clear that given density functional, machine learning offers new ways to learn both the electron density and the corresponding system energy.

Many human-based approaches to improving the approximate functionals in use today rely on imposing physically-motivated constraints. So far, including these types of restrictions on ML-based methods has met with only partial success. For example, requiring that a ML functional fulfill more than one constraint, such as a scaling law and size-consistency, improves overall performance in a system-dependent manner (Hollingsworth et al. 2018). Obtaining accurate derivatives, particularly for molecules with conformational changes, is still an open question for physics-informed ML functionals and potentials that have not been explicitly trained with this goal (Bereau et al. 2018, Snyder et al., 2012).

\section{E. Data set generation}

As for other applications of machine learning, comparison of various methods requires standardized data sets. For quantum chemistry, these include the 134,000 molecules in the QM9 data set (Ramakrishnan et al. 2014) and the COMP6 benchmark data set composed of randomly-sampled subsets of other small molecule and peptide data sets, with each entry optimized using the same computational method (Smith et al. 2018).

In chemistry and materials research, computational data are often expensive to generate, so selection of training data points must be carefully considered. The input and output representations also inform the choice of data. Inspection of ML-predicted molecular energies for most of the QM9 data set showed the importance of choosing input data structures that convey conformer changes (Faber et al. 2017). In addition, dense sampling of the chemical composition space is not always necessary. For example, the initial ANI training set of 20 million molecules could be replaced with 5.5 million training points selected using an active learning method that 


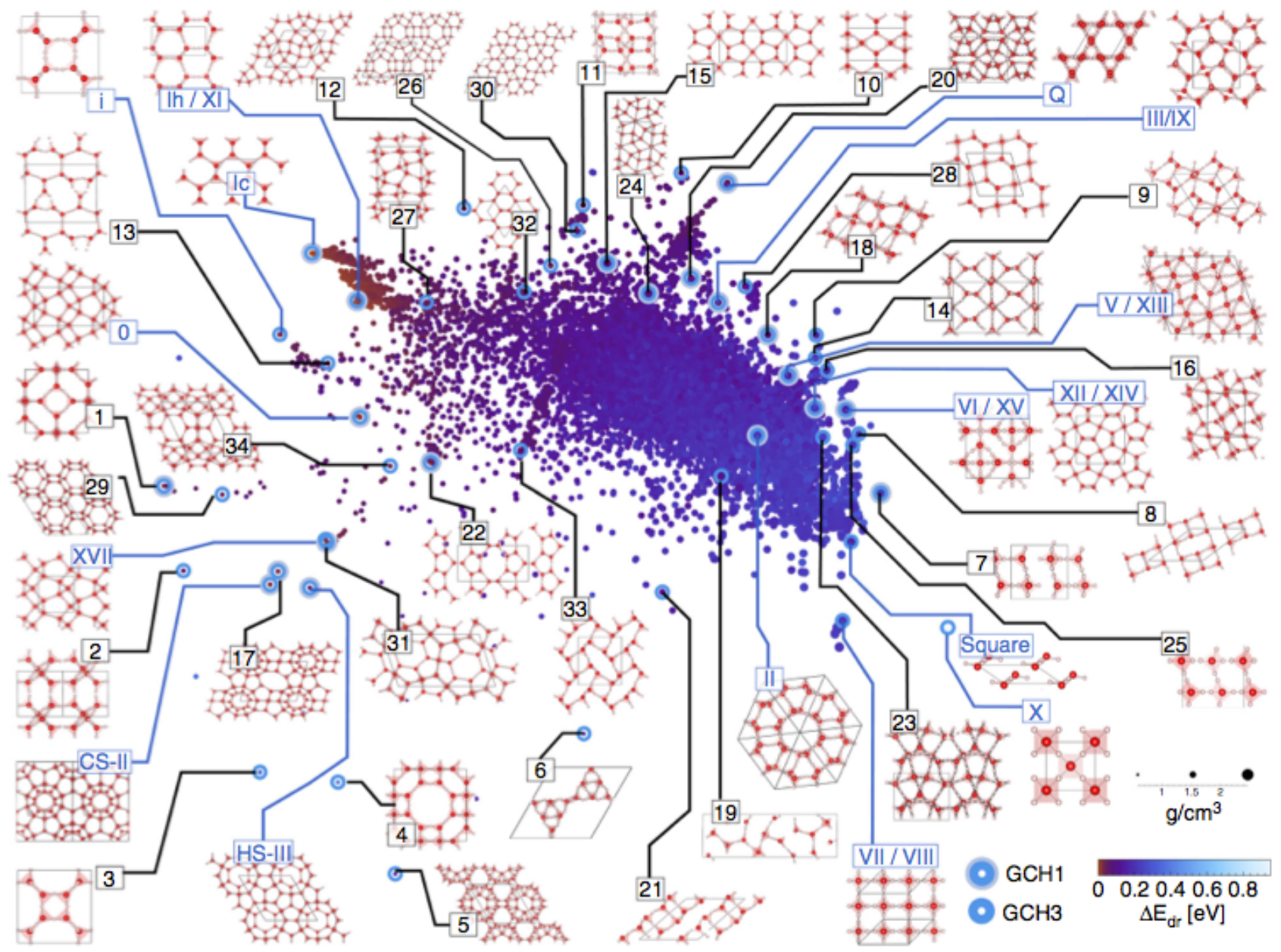

Figure 7 Clustering thousands of possible ice structures based on machine-learned descriptors identifies observed forms and groups similar structures together. Reproduced from (Engel et al. 2018).

added poorly predicted molecular examples from each training cycle (Smith et al. 2018). Alternate sampling approaches can also be used to more efficiently build up a training set. These range from active learning methods that estimate errors from multiple NN evaluations for new molecules (Gastegger et al. 2017) to generating new atomic configurations based on MD simulations using a previously-generated model (Zhang et al. 2018b). Interesting, statistical-physics-based, insight into theoretical aspects of such active learning was presented in (Seung et al. 1992b).

Further work in this area is needed to identify the atomic compositions and configurations that are most important to differentiating candidate structures. While NN's have been shown to generate accurate energies, the amount of data required to prevent over-fitting can be prohibitively expensive in many cases. For specific tasks, such as predicting the anharmonic contributions to vibrational frequencies of the small molecule formaldehye, Gaussian process methods were more accurate, and used fewer points than a NN, although these points need to be selected more carefully (Kamath et al., 2018). Balancing the computational cost of data generation, ease of model training, and model evaluation time continues to be an important consideration when choosing the appropriate ML method for each application.

\section{F. Outlook and Challenges}

Going forward, ML models will benefit from including methods and practices developed for other problems in physics. While some of these ideas are already being explored, such as exploiting input data symmetries for molecular configurations, there are still many opportunities to improve model training efficiency and regularization. Some of the more promising (and challenging) areas include applying methods for exploration of highdimensional landscapes for parameter/hyper-parameter optimization and identifying how to include boundary behaviors or scaling laws in ML architectures and/or in- 
put data formats. To connect more directly to experimental data, future physics-based ML methods should account for uncertainties and/or errors from calculations and measured properties to avoid over-fitting and improve transferability of the models.

\section{AI ACCELERATION WITH CLASSICAL AND QUANTUM HARDWARE}

There are areas where physics can contribute to machine learning by other means than tools for theoretical investigations and domain-specific problems. Novel hardware platforms may help with expensive information processing pipelines and extend the number crunching facilities of CPUs and GPUs. Such hardware-helpers are also known as "AI accelerators", and physics research has to offer a variety of devices that could potentially enhance machine learning.

\section{A. Beyond von Neumann architectures}

When we speak of computers, we usually think of universal digital computers based on electrical circuits and Boolean logic. This is the so-called "von Neumann" paradigm of modern computing. But any physical system can be interpreted as a way to process information, namely by mapping the input parameters of the experimental setup to measurement results, the output. This way of thinking is close to the idea of analog computing, which has been - or so it seems $(\mathrm{Ambs}, 2010$, Lundberg, 2005) - dwarfed by its digital cousin for all but very few applications. In the context of machine learning however, where low-precision computations have to be executed over and over, analog and special-purpose computing devices have found a new surge of interest. The hardware can be used to emulate a full model, such as neural-network inspired chips Ambrogio et al. 2018, or it can outsource only a subroutine of a computation, as done by Field-Programmable Gate Arrays (FPGAs) and Application-Specific Integrated Circuits (ASICs) for fast linear algebra computations (Jouppi et al., 2017, Markidis et al., 2018).

In the following, we present selected examples from various research directions that investigate how hardware platforms from physics labs, such as optics, nanophotonics and quantum computers, can become novel kinds of AI accelerators.

\section{B. Neural networks running on light}

Processing information with optics is a natural and appealing alternative - or at least complement - to all-silicon computers: it is fast, it can be made massively parallel, and requires very low power consumption. Optical interconnects are already widespread, to carry information on short or long distances, but light interference properties also can be leveraged in order to provide more advanced processing. In the case of machine learning there is one more perk. Some of the standard building blocks in optics labs have a striking resemblance with the way information is processed with neural networks (Killoran et al. 2018, Lin et al. 2018, Shen et al., 2017), an insight that is by no means new (Lu et al. 1989). An example for both large bulk optics experiments and on-chip nanophotonics are networks of interferometers. Interferometers are passive optical elements made up of beam splitters and phase shifters (Clements et al. 2016, Reck et al., 1994). If we consider the amplitudes of light modes as an incoming signal, the interferometer effectively applies a unitary transformation to the input (see Figure 8 left). Amplifying or damping the amplitudes can be understood as applying a diagonal matrix. Consequently, by means of a singular value decomposition, an amplifier sandwiched by two interferometers implements an arbitrary matrix multiplication on the data encoded into the optical amplitudes. Adding a non-linear operation - which is usually the hardest to precisely control in the lab - can turn the device into an emulator of a standard neural network layer (Lin et al., 2018, Shen et al., 2017), but at the speed of light.

An interesting question to ask is: what if we use quantum instead of classical light? For example, imagine the information is now encoded in the quadratures of the electromagnetic field. The quadratures are - much like position and momentum of a quantum particle - two noncommuting operators that describe light as a quantum system. We now have to exchange the setup to quantum optics components such as squeezers and displacers, and get a neural network encoded in the quantum properties of light (Killoran et al. . 2018). But there is more: Using multiple layers, and choosing the 'nonlinear operation' as a "non-Gaussian" component (such as an optical "Kerr non-linearity" which is admittedly still an experimental challenge), the optical setup becomes a universal quantum computer. As such, it can run any computations a quantum computer can perform - a true quantum neural network. There are other variations of quantum optical neural nets, for example when information is encoded into discrete rather than continuous-variable properties of light (Steinbrecher et al. 2018 ). Investigations into what these quantum devices mean for machine learning, for example whether there are patterns in data that can be easier recognized, have just begun.

\section{Revealing features in data}

One does not have to implement a full machine learning model on the physical hardware, but can outsource 

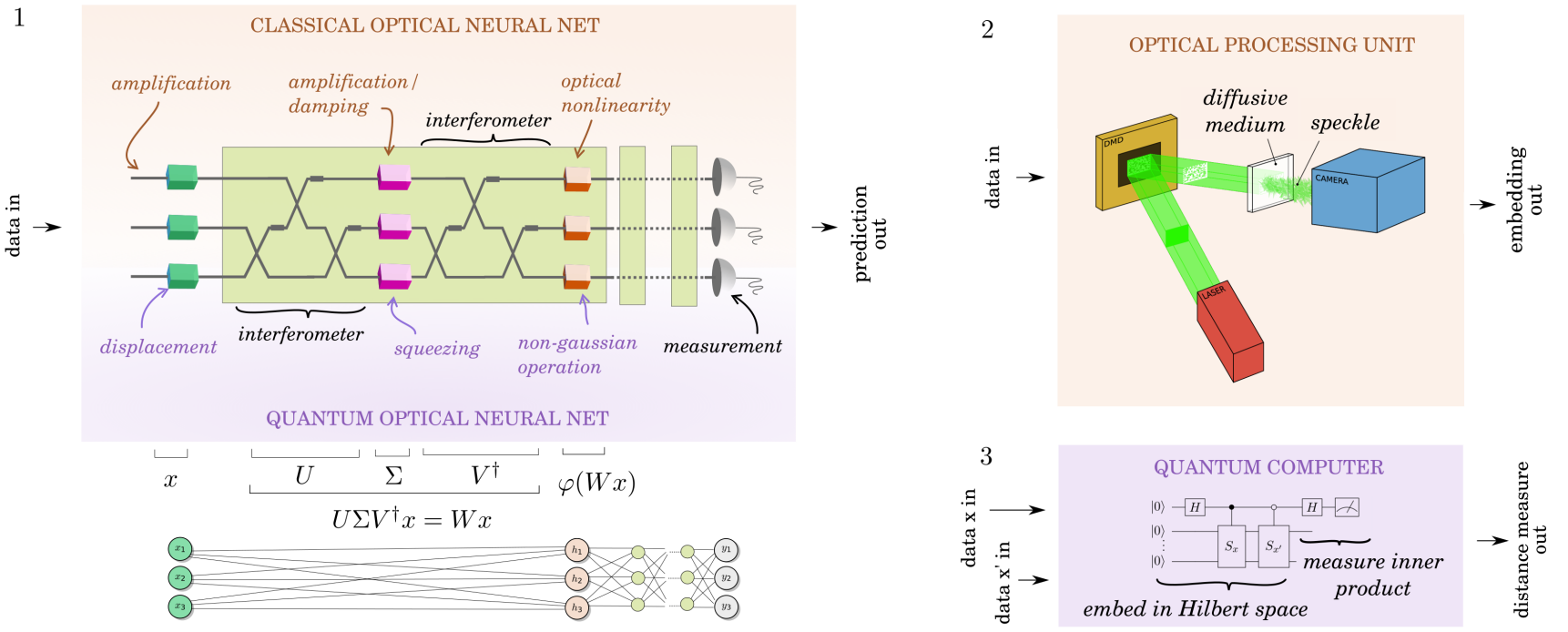

Figure 8 Illustrations of the methods discussed in the text. 1. Optical components such as interferometers and amplifiers can emulate a neural network that layer-wise maps an input $x$ to $\varphi(W x)$ where $W$ is a learnable weight matrix and $\varphi$ a nonlinear activation. Using quantum optics components such as displacement and squeezing, one can encode information into quantum properties of light and turn the neural net into a universal quantum computer. 2. Random embedding with an Optical Processing Unit. Data is encoded into the laser beam through a spatial light modulator (here, a DMD), after which a diffusive medium generates the random features. 3. A quantum computer can be used to compute distances between data points, or "quantum kernels". The first part of the quantum algorithm uses routines $S_{x}, S_{x^{\prime}}$ to embed the data in Hilbert space, while the second part reveals the inner product of the embedded vectors. This kernel can be further processed in standard kernel methods such as support vector machines.

single components. An example which we will highlight as a second application is data preprocessing or feature extraction. This includes mapping data to another space where it is either compressed or 'blown up', in both cases revealing its features for machine learning algorithms.

One approach to data compression or expansion with physical devices leverages the very statistical nature of many machine learning algorithms. Multiple light scattering can generate the very high-dimensional randomness needed for so-called random embeddings (see Figure 8 top right). In a nutshell, the multiplication of a set of vectors by the same random matrix is approximately distance-preserving (Johnson and Lindenstrauss, 1984). This can be used for dimensionality reduction, i.e., data compression, in the spirit of compressed sensing (Donoho, 2006) or for efficient nearest neighbor search with locality sensitive hashing. This can also be used for dimensionality expansion, where in the limit of large dimension it approximates a well-defined kernel (Saade et al., 2016). Such devices can be built in free-space optics, with coherent laser sources, commercial light modulators and CMOS sensors, and a well-chosen scattering material (see Fig 8 2a). Machine learning applications range from transfer learning for deep neural networks, time series analysis - with a feedback loop implementing so-called echo-state networks (Dong et al., 2018), or change-point detection (Keriven et al. . 2018). For large-dimensional data, these devices already outperform CPUs or GPUs both in speed and power consumption.

\section{Quantum-enhanced machine learning}

A fair amount of effort in the field of quantum machine learning, a field that investigates intersections of quantum information and intelligent data mining (Biamonte et al., 2017, Schuld and Petruccione, 2018b), goes into applications of near-term quantum hardware for learning tasks (Perdomo-Ortiz et al., 2017). These so-called Noisy Intermediate-Scale Quantum or 'NISQ' devices are not only hoped to enhance machine learning applications in terms of speed, but may lead to entirely new algorithms inspired by quantum physics. We have already mentioned one such example above, a quantum neural network that can emulate a classical neural net, but go beyond. This model falls into a larger class of variational or parametrized quantum machine learning algorithms (McClean et al., 2016; Mitarai et al., 2018). The idea is to make the quantum algorithm, and thereby the device implementing the quantum computing operations, depend on parameters $\theta$ that can be trained with data. Measurements on the "trained device" represent new outputs, such as artificially generated data samples of a generative model, or classifications of a supervised classifier.

Another idea of how to use quantum computers to en- 
hance learning is inspired by kernel methods (Hofmann et al. 2008) (see Figure 8 bottom right). By associating the parameters of a quantum algorithm with an input data sample $x$, one effectively embeds $x$ into a quantum state $|\psi(x)\rangle$ described by a vector in Hilbert space (Havlicek et al., 2018, Schuld and Killoran, 2018). A simple interference routine can measure overlaps between two quantum states prepared in this way. An overlap is an inner product of vectors in Hilbert space, which in the machine literature is known as a kernel, a distance measure between two data points. As a result, quantum computers can compute rather exotic kernels that may be classically intractable, and it is an active area of research to find interesting quantum kernels for machine learning tasks.

Beyond quantum kernels and variational circuits, quantum machine learning presents many other ideas that use quantum hardware as AI accelerators, for example as a sampler for training and inference in graphical models (Adachi and Henderson, 2015, Benedetti et al. 2017), or for linear algebra computations (Lloyd et al. $2014 \sqrt{2}$ Another interesting branch of research investigates how quantum devices can directly analyze the data produced by quantum experiments, without making the detour of measurements (Cong et al., 2018). In all these explorations, a major challenge is the still severe limitations in current-day NISQ devices which reduce numerical experiments on the hardware to proof-of-principle demonstrations, while theoretical analysis remains notoriously difficult in machine learning.

\section{E. Outlook and Challenges}

The above examples demonstrate a way of how physics research can contribute to machine learning, namely by investigating new hardware platforms to execute tiresome computations. While standard von Neumann technologies struggle to keep pace with Moore's law, this opens a number of opportunities for novel computing paradigms. In their simplest embodiment, these take the form of specialized accelerator devices, plugged onto standard servers and accessed through custom APIs. Future research focuses on the scaling-up of such hardware capabilities, hardware-inspired innovation to machine learning, and adapted programming languages as well as compilers for the optimized distribution of computing tasks on these hybrid servers.

\footnotetext{
2 Many quantum machine learning algorithms based on linear algebra acceleration have recently been shown to make unfounded claims of exponential speedups (Tang, 2018), when compared against classical algorithms for analysing low-rank datasets with strong sampling access. However, they are still interesting in this context where even constant speedups make a difference.
}

\section{CONCLUSIONS AND OUTLOOK}

A number of overarching themes become apparent after reviewing the ways in which machine learning is used in or has enhanced the different disciplines of physics. First of all, it is clear that the interest in machine learning techniques suddenly surged in recent years. This is true even in areas such as statistical physics and highenergy physics where the connection to machine learning techniques has a long history. We are seeing the research move from an exploratory efforts on toy models towards the use of real experimental data. We are also seeing an evolution in the understanding and limitations of these approaches and situations in which the performance can be justified theoretically. A healthy and critical engagement with the potential power and limitations of machine learning includes an analysis of where these methods break and what they are distinctly not good at.

Physicist are notoriously hungry for very detailed understanding of why and when their methods work. As machine learning is incorporated into the physicist's toolbox, it is reasonable to expect that physicist may shed light on some of the notoriously difficult questions machine learning is facing. Specifically, physicists are already contributing to issues of interpretability, techniques to validate or guarantee the results, and principle ways to chose the various parameters of the neural networks architectures.

One direction in which the physics community has much to learn from the machine learning community is the culture and practice of sharing code and developing carefully-crafted, high-quality benchmark datasets. Furthermore, physics would do well to emulate the practices of developing user-friendly and portable implementations of the key methods, ideally with the involvement of professional software engineers.

The picture that emerges from the level of activity and the enthusiasm surrounding the first success stories is that the interaction between machine learning and the physical sciences is merely in its infancy, and we can anticipate more exciting results stemming from this interplay between machine learning and the physical sciences.

\section{ACKNOWLEDGEMENTS}

This research was supported in part by the National Science Foundation under Grants Nos. NSF PHY-1748958, ACI-1450310, OAC-1836650, and DMR1420073 , the US Army Research Office under contract/grant number W911NF-13-1-0387, as well as the ERC under the European Unions Horizon 2020 Research and Innovation Programme Grant Agreement 714608SMiLe. Additionally, we would like to thank the support of the Moore and Sloan foundations, the Kavli Institute of Theoretical Physics of UCSB, and the Institute for Ad- 
vanced Study. Finally, we would like to thank Michele Ceriotti, Yoav Levine, Andrea Rocchetto, Miles Stoudenmire, and Ryan Sweke.

\section{REFERENCES}

Aaronson, S. (2007), Proceedings of the Royal Society A: Mathematical, Physical and Engineering Sciences 463 (2088), 3089.

Aaronson, S. (2017), arXiv:1711.01053 [quant-ph] ArXiv: 1711.01053 .

Acar, E., and B. Yener (2009), IEEE Transactions on Knowledge and Data Engineering 21 (1), 6

Acciarri, R., et al. (MicroBooNE) (2017), JINST 12 (03), P03011, arXiv:1611.05531 [physics.ins-det].

Adachi, S. H., and M. P. Henderson (2015), arXiv preprint arXiv: 1510.06356.

Advani, M. S., and A. M. Saxe (2017), arXiv preprint arXiv:1710.03667.

Agresti, I., N. Viggianiello, F. Flamini, N. Spagnolo, A. Crespi, R. Osellame, N. Wiebe, and F. Sciarrino (2019), Physical Review X 9 (1), 011013.

Albergo, M. S., G. Kanwar, and P. E. Shanahan (2019), Phys. Rev. D100 (3), 034515, arXiv:1904.12072 [hep-lat]

Albertsson, K., et al. (2018), Proceedings, 18th International Workshop on Advanced Computing and Analysis Techniques in Physics Research (ACAT 2017): Seattle, WA, USA, August 21-25, 2017, J. Phys. Conf. Ser. 1085 (2), 022008, arXiv:1807.02876 [physics.comp-ph]

Alet, F., and N. Laflorencie (2018), Comptes Rendus Physique Quantum simulation / Simulation quantique, 19 (6), 498 .

Alsing, J., and B. Wandelt (2019), arXiv:1903.01473 [astroph.CO]

Alsing, J., B. Wandelt, and S. Feeney (2018), Mon. Not. Roy. Astron. Soc. 477 (3), 2874, arXiv:1801.01497 [astroph.CO]

Amari, S.-i. (1998), Neural Computation 10 (2), 251

Ambrogio, S., P. Narayanan, H. Tsai, R. M. Shelby, I. Boybat, C. Nolfo, S. Sidler, M. Giordano, M. Bodini, N. C. Farinha, et al. (2018), Nature 558 (7708), 60.

Ambs, P. (2010), Advances in Optical Technologies 2010.

Amit, D. J., H. Gutfreund, and H. Sompolinsky (1985), Physical Review A 32 (2), 1007.

Anandkumar, A., R. Ge, D. Hsu, S. M. Kakade, and M. Telgarsky (2014), Journal of Machine Learning Research 15, 2773.

Anelli, A., E. A. Engel, C. J. Pickard, and M. Ceriotti (2018), Phys. Rev. Mater. 2, 103804.

Apollinari, G., O. Brüning, T. Nakamoto, and L. Rossi (2015), CERN Yellow Report (5), 1, arXiv:1705.08830 [physics.acc-ph].

Armitage, T. J., S. T. Kay, and D. J. Barnes (2019), MNRAS 484, 1526, arXiv:1810.08430 [astro-ph.CO]

Arsenault, L.-F., R. Neuberg, L. A. Hannah, and A. J. Millis (2017), Inverse Problems 33 (11), 115007

Arunachalam, S., and R. de Wolf (2017), ACM SIGACT News 48 (2), 41.

ATLAS Collaboration, (2018), Deep generative models for fast shower simulation in ATLAS, Tech. Rep. ATL-SOFTPUB-2018-001 (CERN, Geneva).
Aubin, B., A. Maillard, F. Krzakala, N. Macris, L. Zdeborová, et al. (2018), in Advances in Neural Information Processing Systems, pp. 3223-3234, preprint arXiv:1806.05451.

Aurisano, A., A. Radovic, D. Rocco, A. Himmel, M. D. Messier, E. Niner, G. Pawloski, F. Psihas, A. Sousa, and P. Vahle (2016), JINST 11 (09), P09001, arXiv:1604.01444 [hep-ex].

Baireuther, P., T. E. O'Brien, B. Tarasinski, and C. W. J. Beenakker (2018), Quantum 2, 48.

Baity-Jesi, M., L. Sagun, M. Geiger, S. Spigler, G. B. Arous, C. Cammarota, Y. LeCun, M. Wyart, and G. Biroli (2018), in ICML 2018, arXiv preprint arXiv:1803.06969.

Baldassi, C., C. Borgs, J. T. Chayes, A. Ingrosso, C. Lucibello, L. Saglietti, and R. Zecchina (2016), Proceedings of the National Academy of Sciences 113 (48), E7655.

Baldassi, C., A. Ingrosso, C. Lucibello, L. Saglietti, and R. Zecchina (2015), Physical review letters 115 (12), 128101.

Baldi, P., K. Bauer, C. Eng, P. Sadowski, and D. Whiteson (2016a), Physical Review D 93 (9), 094034.

Baldi, P., K. Cranmer, T. Faucett, P. Sadowski, and D. Whiteson (2016b), Eur. Phys. J. C76 (5), 235 , arXiv:1601.07913 [hep-ex]

Baldi, P., P. Sadowski, and D. Whiteson (2014), Nature Commun. 5, 4308, arXiv:1402.4735 [hep-ph]

Ball, R. D., et al. (NNPDF) (2015), JHEP 04, 040, arXiv:1410.8849 [hep-ph].

Ballard, A. J., R. Das, S. Martiniani, D. Mehta, L. Sagun, J. D. Stevenson, and D. J. Wales (2017), Phys. Chem. Chem. Phys. 19 (20), 12585.

Banchi, L., E. Grant, A. Rocchetto, and S. Severini (2018), New Journal of Physics 20 (12), 123030.

Bang, J., J. Ryu, S. Yoo, M. Pawłowski, and J. Lee (2014), New Journal of Physics 16 (7), 073017.

Barbier, J., M. Dia, N. Macris, F. Krzakala, T. Lesieur, and L. Zdeborová (2016), in Advances in Neural Information Processing Systems, pp. 424-432.

Barbier, J., F. Krzakala, N. Macris, L. Miolane, and L. Zdeborová (2019), Proceedings of the National Academy of Sciences 116 (12), 5451.

Barkai, N., and H. Sompolinsky (1994), Physical Review E 50 (3), 1766.

Barra, A., G. Genovese, P. Sollich, and D. Tantari (2018), Physical Review E 97 (2), 022310.

Bartók, A. P., J. Kermode, N. Bernstein, and G. Csányi (2018), Physical Review X 8 (4), 041048.

Bartók, A. P., R. Kondor, and G. Csányi (2013), Phys. Rev. B 87, 184115 .

Bartók, A. P., M. C. Payne, R. Kondor, and G. Csányi (2010), Phys. Rev. Lett. 104 (13), 136403.

Baydin, A. G., L. Heinrich, W. Bhimji, B. Gram-Hansen, G. Louppe, L. Shao, Prabhat, K. Cranmer, and F. Wood (2018), arXiv:1807.07706 [cs.LG]

Beach, M. J. S., A. Golubeva, and R. G. Melko (2018), Physical Review B 97 (4), 045207.

Beaumont, M. A., W. Zhang, and D. J. Balding (2002), Genetics 162 (4), 2025.

Becca, F., and S. Sorella (2017), Quantum Monte Carlo Approaches for Correlated Systems (Cambridge University Press, Cambridge, United Kingdom ; New York, NY).

Behler, J. (2016), J. Chem. Phys. 145 (17), 170901.

Behler, J., and M. Parrinello (2007), Phys. Rev. Lett. 98 (14), 583. 
Benedetti, M., J. Realpe-Gómez, R. Biswas, and A. PerdomoOrtiz (2017), Physical Review X 7 (4), 041052.

Benítez, N. (2000), Astrophys. J. 536, 571, arXiv:astro$\mathrm{ph} / 9811189$ [astro-ph].

Bény, C. (2013), in ICLR 2013, arXiv preprint arXiv:1301.3124.

Bereau, T., R. A. DiStasio, Jr., A. Tkatchenko, and O. A. von Lilienfeld (2018), J. Chem. Phys. 148 (24), 241706.

Biamonte, J., P. Wittek, N. Pancotti, P. Rebentrost, N. Wiebe, and S. Lloyd (2017), Nature 549 (7671), 195.

Biehl, M., and A. Mietzner (1993), EPL (Europhysics Letters) $24(5), 421$.

Bishop, C. M. (2006), Pattern recognition and machine learning (springer).

Bohrdt, A., C. S. Chiu, G. Ji, M. Xu, D. Greif, M. Greiner, E. Demler, F. Grusdt, and M. Knap (2018), arXiv:1811.12425 [cond-mat]

Bolthausen, E. (2014), Communications in Mathematical Physics 325 (1), 333.

Bonnett, C., et al. (DES) (2016), Phys. Rev. D94 (4), 042005 arXiv:1507.05909 [astro-ph.CO|.

Borin, A., and D. A. Abanin (2019), arXiv:1901.08615 [condmat, physics:quant-ph].

Bozson, A., G. Cowan, and F. Spanò (2018), arXiv:1811.01242 [physics.data-an]

Bradde, S., and W. Bialek (2017), Journal of Statistical Physics 167 (3-4), 462.

Brammer, G. B., P. G. van Dokkum, and P. Coppi (2008), Astrophys. J. 686, 1503, arXiv:0807.1533 [astro-ph].

Brehmer, J., K. Cranmer, G. Louppe, and J. Pavez (2018a), Phys. Rev. D98 (5), 052004, arXiv:1805.00020 [hep-ph].

Brehmer, J., K. Cranmer, G. Louppe, and J. Pavez (2018b), Phys. Rev. Lett. 121 (11), 111801, arXiv:1805.00013 [hepph]

Brehmer, J., G. Louppe, J. Pavez, and K. Cranmer (2018c), arXiv:1805.12244 [stat.ML].

Breiman, L., J. H. Friedman, R. A. Olshen, and C. J. Stone (1984).

Brockherde, F., L. Vogt, L. Li, M. E. Tuckerman, K. Burke, and K.-R. Müller (2017), Nat. Commun. 8 (1), 872.

Broecker, P., F. F. Assaad, and S. Trebst (2017a), arXiv:1707.00663 [cond-mat]

Broecker, P., J. Carrasquilla, R. G. Melko, and S. Trebst (2017b), Scientific Reports 7 (1), 8823

Bronstein, M. M., J. Bruna, Y. LeCun, A. Szlam, and P. Vandergheynst (2017), IEEE Sig. Proc. Mag. 34 (4), 18, arXiv:1611.08097 [cs.CV]

Bukov, M. (2018), Physical Review B 98 (22), 224305.

Bukov, M., A. G. Day, D. Sels, P. Weinberg, A. Polkovnikov, and P. Mehta (2018), Physical Review X 8 (3), 031086

Butler, K. T., D. W. Davies, H. Cartwright, O. Isayev, and A. Walsh (2018), Nature 559, 547.

Cai, Z., and J. Liu (2018), Physical Review B 97 (3), 035116

Cameron, E., and A. N. Pettitt (2012), MNRAS 425, 44, arXiv:1202.1426 [astro-ph.IM]

Carifio, J., J. Halverson, D. Krioukov, and B. D. Nelson (2017), JHEP 09, 157, arXiv:1707.00655 [hep-th].

Carleo, G., F. Becca, M. Schiro, and M. Fabrizio (2012), Scientific Reports 2, 243.

Carleo, G., Y. Nomura, and M. Imada (2018), Nature communications 9 (1), 5322.

Carleo, G., and M. Troyer (2017), Science 355 (6325), 602.

Carrasco Kind, M., and R. J. Brunner (2013), MNRAS 432, 1483, arXiv:1303.7269 [astro-ph.CO]
Carrasquilla, J., and R. G. Melko (2017), Nature Physics 13 (5), 431.

Carrasquilla, J., G. Torlai, R. G. Melko, and L. Aolita (2019), Nature Machine Intelligence 1 (3), 155.

Casado, M. L., et al. (2017), arXiv:1712.07901 [cs.AI],

Changlani, H. J., J. M. Kinder, C. J. Umrigar, and G. K.-L. Chan (2009), Physical Review B 80 (24), 245116

Charnock, T., G. Lavaux, and B. D. Wandelt (2018), Phys. Rev. D97 (8), 083004, arXiv:1802.03537 [astro-ph.IM].

Chaudhari, P., A. Choromanska, S. Soatto, Y. LeCun, C. Baldassi, C. Borgs, J. Chayes, L. Sagun, and R. Zecchina (2016), in ICLR 2017, arXiv preprint arXiv:1611.01838.

Chen, C., X. Y. Xu, J. Liu, G. Batrouni, R. Scalettar, and Z. Y. Meng (2018a), Physical Review B 98 (4), 041102

Chen, J., S. Cheng, H. Xie, L. Wang, and T. Xiang (2018b), Physical Review B 97 (8), 085104.

Cheng, S., L. Wang, T. Xiang, and P. Zhang (2019), arXiv:1901.02217 [cond-mat, physics:quant-ph, stat].

Chmiela, S., H. E. Sauceda, K.-R. Müller, and A. Tkatchenko (2018), Nat. Commun. 9, 3887.

Choma, N., F. Monti, L. Gerhardt, T. Palczewski, Z. Ronaghi, Prabhat, W. Bhimji, M. M. Bronstein, S. R. Klein, and J. Bruna (2018), arXiv e-prints , arXiv:1809.06166arXiv:1809.06166 [cs.LG]

Choo, K., G. Carleo, N. Regnault, and T. Neupert (2018), Physical Review Letters 121 (16), 167204.

Choromanska, A., M. Henaff, M. Mathieu, G. B. Arous, and Y. LeCun (2015), in Artificial Intelligence and Statistics, pp. $192-204$.

Chung, S., D. D. Lee, and H. Sompolinsky (2018), Physical Review X 8 (3), 031003.

Ciliberto, C., M. Herbster, A. D. Ialongo, M. Pontil, A. Rocchetto, S. Severini, and L. Wossnig (2018), Proceedings of the Royal Society A: Mathematical, Physical and Engineering Sciences 474 (2209), 20170551.

Clark, S. R. (2018), Journal of Physics A: Mathematical and Theoretical 51 (13), 135301.

Clements, W. R., P. C. Humphreys, B. J. Metcalf, W. S. Kolthammer, and I. A. Walmsley (2016), Optica 3 (12), 1460.

Cocco, S., R. Monasson, L. Posani, S. Rosay, and J. Tubiana (2018), Physica A: Statistical Mechanics and its Applications 504, 45.

Cohen, N., O. Sharir, and A. Shashua (2016), in 29th Annual Conference on Learning Theory, Proceedings of Machine Learning Research, Vol. 49, edited by V. Feldman, A. Rakhlin, and O. Shamir (PMLR, Columbia University, New York, New York, USA) pp. 698-728.

Cohen, T., and M. Welling (2016), in International conference on machine learning, pp. 2990-2999.

Cohen, T. S., M. Geiger, J. Köhler, and M. Welling (2018), Proceedings of the 6th International Conference on Learning Representations (ICLR) ArXiv preprint arXiv:1801.10130.

Cohen, T. S., M. Weiler, B. Kicanaoglu, and M. Welling (2019), arXiv e-prints arXiv:1902.04615 [cs.LG]

Coja-Oghlan, A., F. Krzakala, W. Perkins, and L. Zdeborová (2018), Advances in Mathematics 333, 694.

Collett, T. E. (2015), The Astrophysical Journal 811 (1), 20

Collister, A. A., and O. Lahav (2004), Publications of the Astronomical Society of the Pacific 116, 345, arXiv:astro$\mathrm{ph} / 0311058$ [astro-ph].

Cong, I., S. Choi, and M. D. Lukin (2018), arXiv preprint arXiv:1810.03787. 
Cranmer, K., S. Golkar, and D. Pappadopulo (2019), arXiv:1904.05903 [hep-lat, physics:physics, physics:quantph, stat] ArXiv: 1904.05903.

Cranmer, K., and G. Louppe (2016), J. Brief Ideas 10.5281/zenodo.198541.

Cranmer, K., J. Pavez, and G. Louppe (2015), arXiv:1506.02169 [stat.AP]

Cristoforetti, M., G. Jurman, A. I. Nardelli, and C. Furlanello (2017), arXiv:1705.09524 [cond-mat, physics:hep-lat].

Cubuk, E. D., S. S. Schoenholz, J. M. Rieser, B. D. Malone, J. Rottler, D. J. Durian, E. Kaxiras, and A. J. Liu (2015), Physical review letters 114 (10), 108001.

Cybenko, G. (1989), Mathematics of control, signals and systems 2 (4), 303.

Czischek, S., M. Gärttner, and T. Gasenzer (2018), Physical Review B 98 (2), 024311.

Dean, D. S. (1996), Journal of Physics A: Mathematical and General 29 (24), L613.

Decelle, A., G. Fissore, and C. Furtlehner (2017), EPL (Europhysics Letters) $\mathbf{1 1 9}$ (6), 60001.

Decelle, A., F. Krzakala, C. Moore, and L. Zdeborová (2011a), Physical Review E 84 (6), 066106.

Decelle, A., F. Krzakala, C. Moore, and L. Zdeborová (2011b), Physical Review Letters 107 (6), 065701.

Deng, D.-L., X. Li, and S. Das Sarma (2017a), Physical Review B 96 (19), 195145.

Deng, D.-L., X. Li, and S. Das Sarma (2017b), Physical Review X 7 (2), 021021

Deringer, V. L., N. Bernstein, A. P. Bartók, M. J. Cliffe, R. N. Kerber, L. E. Marbella, C. P. Grey, S. R. Elliott, and G. Csányi (2018), J. Phys. Chem. Lett. 9 (11), 2879.

Deshpande, Y., and A. Montanari (2014), in 2014 IEEE International Symposium on Information Theory.

Dirac, P. A. M. (1930), Mathematical Proceedings of the Cambridge Philosophical Society 26 (03), 376

Doggen, E. V. H., F. Schindler, K. S. Tikhonov, A. D. Mirlin, T. Neupert, D. G. Polyakov, and I. V. Gornyi (2018), Physical Review B 98 (17), 174202

Dong, J., S. Gigan, F. Krzakala, and G. Wainrib (2018), in 2018 IEEE Statistical Signal Processing Workshop (SSP) (IEEE) pp. 448-452.

Donoho, D. L. (2006), IEEE Transactions on information theory $\mathbf{5 2}(4), 1289$.

Duarte, J., et al. (2018), JINST 13 (07), P07027, arXiv:1804.06913 [physics.ins-det]

Dunjko, V., and H. J. Briegel (2018), Reports on Progress in Physics 81 (7), 074001.

Duvenaud, D., J. Lloyd, R. Grosse, J. Tenenbaum, and G. Zoubin (2013), in International Conference on Machine Learning, pp. 1166-1174, 1302.4922.

Eickenberg, M., G. Exarchakis, M. Hirn, S. Mallat, and L. Thiry (2018), J. Chem. Phys. 148 (24), 241732.

Engel, A., and C. Van den Broeck (2001), Statistical mechanics of learning (Cambridge University Press).

Engel, E. A., A. Anelli, M. Ceriotti, C. J. Pickard, and R. J. Needs (2018), Nat. Commun. 9, 2173.

Estrada, J., J. Annis, H. T. Diehl, P. B. Hall, T. Las, H. Lin, M. Makler, K. W. Merritt, V. Scarpine, S. Allam, and D. Tucker (2007), Astrophys. J. 660, 1176, astro$\mathrm{ph} / 0701383$.

Faber, F. A., L. Hutchison, B. Huang, J. Gilmer, S. S. Schoenholz, G. E. Dahl, O. Vinyals, S. Kearnes, P. F. Riley, and O. A. von Lilienfeld (2017), J. Chem. Theory Comput. 13 (11), 5255.
Fabiani, G., and J. Mentink (2019), SciPost Physics 7 (1), 004

Farrell, S., et al. (2018), in 4th International Workshop Connecting The Dots 2018 (CTD2018) Seattle, Washington, USA, March 20-22, 2018, arXiv:1810.06111 [hep-ex]

Feldmann, R., C. M. Carollo, C. Porciani, S. J. Lilly, P. Capak, Y. Taniguchi, O. Le Fèvre, A. Renzini, N. Scoville, M. Ajiki, H. Aussel, T. Contini, H. McCracken, B. Mobasher, T. Murayama, D. Sanders, S. Sasaki, C. Scarlata, M. Scodeggio, Y. Shioya, J. Silverman, M. Takahashi, D. Thompson, and G. Zamorani (2006), MNRAS 372, 565 , arXiv:astro-ph/0609044 [astro-ph]

Firth, A. E., O. Lahav, and R. S. Somerville (2003), MNRAS 339, 1195, arXiv:astro-ph/0203250 [astro-ph]

Foreman-Mackey, D., D. W. Hogg, D. Lang, and J. Goodman (2013), Publ.Astron.Soc.Pac. 125, 306, arXiv:1202.3665 [astro-ph.IM]

Forte, S., L. Garrido, J. I. Latorre, and A. Piccione (2002), JHEP 05, 062, arXiv:hep-ph/0204232 [hep-ph]

Fortunato, S. (2010), Physics reports 486 (3-5), 75.

Fournier, R., L. Wang, O. V. Yazyev, and Q. Wu (2018), arXiv:1810.00913 [cond-mat, physics:physics].

Frate, M., K. Cranmer, S. Kalia, A. Vandenberg-Rodes, and D. Whiteson (2017), arXiv:1709.05681 [physics.data-an]

Frazier, P. I. (2018), arXiv preprint arXiv:1807.02811.

Frenkel, Y. I. (1934), Wave Mechanics: Advanced General Theory, The International series of monographs on nuclear energy: Reactor design physics No. v. 2 (The Clarendon Press).

Freund, Y., and R. E. Schapire (1997), J. Comput. Syst. Sci. 55 (1), 119.

Fösel, T., P. Tighineanu, T. Weiss, and F. Marquardt (2018), Physical Review X 8 (3), 031084.

Gabrié, M., A. Manoel, C. Luneau, N. Macris, F. Krzakala, L. Zdeborová, et al. (2018), in Advances in Neural Information Processing Systems, pp. 1821-1831, preprint arXiv:1805.09785.

Gabrié, M., E. W. Tramel, and F. Krzakala (2015), in Advances in Neural Information Processing Systems, pp. 640648.

Gao, X., and L.-M. Duan (2017), Nature Communications $8(1), 662$.

Gardner, E. (1987), EPL (Europhysics Letters) 4 (4), 481.

Gardner, E. (1988), Journal of physics A: Mathematical and general 21 (1), 257.

Gardner, E., and B. Derrida (1989), Journal of Physics A: Mathematical and General 22 (12), 1983.

Gastegger, M., J. Behler, and P. Marquetand (2017), Chem. Sci. 8 (10), 6924.

Gendiar, A., and T. Nishino (2002), Physical Review E 65 (4), 046702.

Glasser, I., N. Pancotti, M. August, I. D. Rodriguez, and J. I. Cirac (2018a), Physical Review X 8 (1), 011006.

Glasser, I., N. Pancotti, and J. I. Cirac (2018b), arXiv preprint arXiv:1806.05964.

Gligorov, V. V., and M. Williams (2013), JINST 8, P02013, arXiv:1210.6861 [physics.ins-det],

Goldt, S., M. S. Advani, A. M. Saxe, F. Krzakala, and L. Zdeborová (2019a), arXiv preprint arXiv:1906.08632.

Goldt, S., M. S. Advani, A. M. Saxe, F. Krzakala, and L. Zdeborová (2019b), arXiv preprint arXiv:1901.09085.

Golkar, S., and K. Cranmer (2018), arXiv:1806.01337 [stat.ML]. 
Goodfellow, I., Y. Bengio, and A. Courville (2016), Deep learning (MIT press).

Goodfellow, I., J. Pouget-Abadie, M. Mirza, B. Xu, D. WardeFarley, S. Ozair, A. Courville, and Y. Bengio (2014), in Advances in neural information processing systems, pp. 26722680.

Gorodetsky, A., S. Karaman, and Y. Marzouk (2019), Computer Methods in Applied Mechanics and Engineering 347, 59.

Gray, J., L. Banchi, A. Bayat, and S. Bose (2018), Physical Review Letters 121 (15), 150503.

Greitemann, J., K. Liu, L. Pollet, et al. (2019), Physical Review B 99 (6), 060404.

Gross, D., Y.-K. Liu, S. T. Flammia, S. Becker, and J. Eisert (2010), Physical Review Letters 105 (15), 150401.

Guest, D., J. Collado, P. Baldi, S.-C. Hsu, G. Urban, and D. Whiteson (2016), Phys. Rev. D94 (11), 112002 arXiv:1607.08633 [hep-ex].

Guest, D., K. Cranmer, and D. Whiteson (2018), Ann. Rev. Nucl. Part. Sci. 68, 161, arXiv:1806.11484 [hep-ex].

Guo, C., Z. Jie, W. Lu, and D. Poletti (2018), Physical Review E 98 (4), 042114

Györgyi, G. (1990), Physical Review A 41 (12), 7097.

Györgyi, G., and N. Tishby (1990), in W.T. Theumann and R. Kobrele (Editors), Neural Networks and Spin Glasses, 3.

Haah, J., A. W. Harrow, Z. Ji, X. Wu, and N. Yu (2017), IEEE Transactions on Information Theory 63 (9), 5628.

Hackbusch, W., and S. Kühn (2009), Journal of Fourier Analysis and Applications 15 (5), 706 .

Han, J., L. Zhang, and W. E (2018a), arXiv:1807.07014 [physics]

Han, Z.-Y., J. Wang, H. Fan, L. Wang, and P. Zhang (2018b), Physical Review X 8 (3), 031012

Hartmann, M. J., and G. Carleo (2019), arXiv:1902.05131 [cond-mat, physics:quant-ph].

Hashimoto, K., S. Sugishita, A. Tanaka, and A. Tomiya (2018a), Phys. Rev. D 98, 106014.

Hashimoto, K., S. Sugishita, A. Tanaka, and A. Tomiya (2018b), Phys. Rev. D98 (4), 046019, arXiv:1802.08313 [hep-th]

Havlicek, V., A. D. Córcoles, K. Temme, A. W. Harrow, J. M. Chow, and J. M. Gambetta (2018), arXiv preprint arXiv:1804.11326.

He, S., Y. Li, Y. Feng, S. Ho, S. Ravanbakhsh, W. Chen, and B. Póczos (2018), arXiv e-prints arXiv:1811.06533 [astroph.CO]

Henson, M. A., S. T. Kay, D. J. Barnes, I. G. McCarthy, J. Schaye, and A. Jenkins (2016), Monthly Notices of the Royal Astronomical Society 465 (1), 213, http://oup.prod.sis.lan/mnras/articlepdf $/ 465 / 1 / 213 / 8593372 /$ stw2722.pdf

Hermans, J., V. Begy, and G. Louppe (2019), arXiv e-prints arXiv:1903.04057 [stat.ML],

Hezaveh, Y. D., L. Perreault Levasseur, and P. J. Marshall (2017), Nature 548, 555, arXiv:1708.08842 [astro-ph.IM]

Hinton, G. E. (2002), Neural computation 14 (8), 1771.

Ho, M., M. M. Rau, M. Ntampaka, A. Farahi, H. Trac, and B. Poczos (2019), arXiv:1902.05950 [astro-ph.CO]

Hochreiter, S., and J. Schmidhuber (1997), Neural computation 9 (8), 1735.

Hofmann, T., B. Schölkopf, and A. J. Smola (2008), The Annals of Statistics, 1171.
Hollingsworth, J., T. E. Baker, and K. Burke (2018), J. Chem. Phys. 148 (24), 241743.

Hopfield, J. J. (1982), Proceedings of the national academy of sciences 79 (8), 2554.

Hsu, Y.-T., X. Li, D.-L. Deng, and S. Das Sarma (2018), Physical Review Letters 121 (24), 245701.

Hu, W., R. R. P. Singh, and R. T. Scalettar (2017), Physical Review E 95 (6), 062122.

Huang, L., and L. Wang (2017), Physical Review B 95 (3), 035105

Huang, Y., and J. E. Moore (2017), arXiv:1701.06246 [condmat]

Huembeli, P., A. Dauphin, and P. Wittek (2018a), Physical Review B 97 (13), 134109.

Huembeli, P., A. Dauphin, P. Wittek, and C. Gogolin (2018b), arXiv:1806.00419 [cond-mat, physics:quant-ph] ArXiv: 1806.00419.

Iakovlev, I., O. Sotnikov, and V. Mazurenko (2018), Physical Review B 98 (17), 174411.

Ilten, P., M. Williams, and Y. Yang (2017), JINST 12 (04), P04028, arXiv:1610.08328 [physics.data-an]

Ishida, E. E. O., S. D. P. Vitenti, M. Penna-Lima, J. Cisewski, R. S. de Souza, A. M. M. Trindade, E. Cameron, V. C. Busti, and COIN Collaboration (2015), Astronomy and Computing 13, 1, arXiv:1504.06129 [astro-ph.CO]

Izmailov, P., A. Novikov, and D. Kropotov (2017), arXiv:1710.07324 [cs, stat]

Jacot, A., F. Gabriel, and C. Hongler (2018), in Advances in neural information processing systems, pp. 8580-8589.

Jaeger, H., and H. Haas (2004), science 304 (5667), 78.

Jain, A., P. K. Srijith, and S. Desai (2018), arXiv:1803.06473 [astro-ph.IM],

Javanmard, A., and A. Montanari (2013), Information and Inference: A Journal of the IMA 2 (2), 115.

Johnson, W. B., and J. Lindenstrauss (1984), Contemporary mathematics 26 (189-206), 1.

Johnstone, I. M., and A. Y. Lu (2009), Journal of the American Statistical Association 104 (486), 682.

Jones, D. R., M. Schonlau, and W. J. Welch (1998), Journal of Global Optimization 13 (4), 455

Jouppi, N. P., C. Young, N. Patil, D. Patterson, G. Agrawal, R. Bajwa, S. Bates, S. Bhatia, N. Boden, A. Borchers, et al. (2017), in Computer Architecture (ISCA), 2017 ACM/IEEE 44th Annual International Symposium on (IEEE) pp. 1-12.

Jónsson, B., B. Bauer, and G. Carleo (2018), arXiv:1808.05232 [cond-mat, physics:physics, physics:quant-ph]

Kabashima, Y., F. Krzakala, M. Mézard, A. Sakata, and L. Zdeborová (2016), IEEE Transactions on Information Theory 62 (7), 4228.

Kalantre, S. S., J. P. Zwolak, S. Ragole, X. Wu, N. M. Zimmerman, M. Stewart, and J. M. Taylor (2019), npj Quantum Information 5 (1), 6.

Kamath, A., R. A. Vargas-Hernández, R. V. Krems, T. Carrington, Jr, and S. Manzhos (2018), J. Chem. Phys. 148 (24), 241702.

Kashiwa, K., Y. Kikuchi, and A. Tomiya (2019), Progress of Theoretical and Experimental Physics 2019 (8), 083A04.

Kasieczka, G., T. Plehn, A. Butter, D. Debnath, M. Fairbairn, W. Fedorko, C. Gay, L. Gouskos, P. Komiske, S. Leiss, et al. (2019), arXiv:1902.09914 [hep-ph].

Kaubruegger, R., L. Pastori, and J. C. Budich (2018), Physical Review B 97 (19), 195136 
Keriven, N., D. Garreau, and I. Poli (2018), arXiv preprint arXiv:1805.08061.

Killoran, N., T. R. Bromley, J. M. Arrazola, M. Schuld, N. Quesada, and S. Lloyd (2018), "Continuous-variable quantum neural networks," arxiv:1806.06871

Kingma, D. P., and M. Welling (2013), arXiv preprint arXiv:1312.6114.

Koch-Janusz, M., and Z. Ringel (2018), Nature Physics 14 (6), 578.

Kochkov, D., and B. K. Clark (2018), arXiv:1811.12423 [cond-mat, physics:physics]

Komiske, P. T., E. M. Metodiev, B. Nachman, and M. D. Schwartz (2018a), Phys. Rev. D98 (1), 011502 arXiv:1801.10158 [hep-ph]

Komiske, P. T., E. M. Metodiev, and J. Thaler (2018b), JHEP 04, 013, arXiv:1712.07124 [hep-ph]

Komiske, P. T., E. M. Metodiev, and J. Thaler (2019), JHEP 01, 121, arXiv:1810.05165 [hep-ph]

Kondor, R. (2018), CoRR abs/1803.01588 arXiv:1803.01588.

Kondor, R., Z. Lin, and S. Trivedi (2018), NeurIPS 2018 , arXiv:1806.09231arXiv:1806.09231 [stat.ML].

Kondor, R., and S. Trivedi (2018), in International Conference on Machine Learning, pp. 2747-2755, 1802.03690.

Krastanov, S., and L. Jiang (2017), Scientific reports 7 (1), 11003.

Krenn, M., M. Malik, R. Fickler, R. Lapkiewicz, and A. Zeilinger (2016), Physical review letters 116 (9), 090405.

Krzakala, F., M. Mézard, and L. Zdeborová (2013a), in Information Theory Proceedings (ISIT), 2013 IEEE International Symposium on (IEEE) pp. 659-663.

Krzakala, F., C. Moore, E. Mossel, J. Neeman, A. Sly, L. Zdeborová, and P. Zhang (2013b), Proceedings of the National Academy of Sciences 110 (52), 20935.

Lang, D., D. W. Hogg, and D. Mykytyn (2016), "The Tractor: Probabilistic astronomical source detection and measurement," Astrophysics Source Code Library, ascl:1604.008.

Lanusse, F., Q. Ma, N. Li, T. E. Collett, C.-L. Li, S. Ravanbakhsh, R. Mandelbaum, and B. Póczos (2018), MNRAS 473, 3895, arXiv:1703.02642 [astro-ph.IM]

Lanyon, B. P., C. Maier, M. Holzäpfel, T. Baumgratz, C. Hempel, P. Jurcevic, I. Dhand, A. S. Buyskikh, A. J. Daley, M. Cramer, M. B. Plenio, R. Blatt, and C. F. Roos (2017), Nature Physics advance online publication, $10.1038 /$ nphys 4244 .

Larkoski, A. J., I. Moult, and B. Nachman (2017), arXiv:1709.04464 [hep-ph]

Larochelle, H., and I. Murray (2011), in Proceedings of the Fourteenth International Conference on Artificial Intelligence and Statistics, pp. 29-37.

Le, T. A., A. G. Baydin, and F. Wood (2017), in Artificial Intelligence and Statistics, pp. 1338-1348, arXiv:1610.09900

LeCun, Y., Y. Bengio, and G. Hinton (2015), nature $\mathbf{5 2 1}$ (7553), 436.

Lee, J., Y. Bahri, R. Novak, S. S. Schoenholz, J. Pennington, and J. Sohl-Dickstein (2018), ICRL 2018 arXiv:1711.00165

Leistedt, B., D. W. Hogg, R. H. Wechsler, and J. DeRose (2018), arXiv e-prints , arXiv:1807.01391 arXiv:1807.01391 [astro-ph.CO]

Lelarge, M., and L. Miolane (2016), Probability Theory and Related Fields, 1.

Levine, Y., O. Sharir, N. Cohen, and A. Shashua (2019), Physical Review Letters 122 (6), 065301.
Levine, Y., D. Yakira, N. Cohen, and A. Shashua (2017), ICLR 2018 ArXiv: 1704.01552.

Li, L., J. C. Snyder, I. M. Pelaschier, J. Huang, U.-N. Niranjan, P. Duncan, M. Rupp, K.-R. Müller, and K. Burke (2015), Int. J. Quantum Chem. 116 (11), 819.

Li, N., M. D. Gladders, E. M. Rangel, M. K. Florian, L. E. Bleem, K. Heitmann, S. Habib, and P. Fasel (2016), The Astrophysical Journal 828 (1), 54.

Li, S.-H., and L. Wang (2018), Phys. Rev. Lett. 121, 260601.

Liang, X., W.-Y. Liu, P.-Z. Lin, G.-C. Guo, Y.-S. Zhang, and L. He (2018), Physical Review B 98 (10), 104426

Likhomanenko, T., P. Ilten, E. Khairullin, A. Rogozhnikov, A. Ustyuzhanin, and M. Williams (2015), Proceedings, 21st International Conference on Computing in High Energy and Nuclear Physics (CHEP 2015): Okinawa, Japan, April 13-17, 2015, J. Phys. Conf. Ser. 664 (8), 082025. arXiv:1510.00572 [physics.ins-det]

Lin, X., Y. Rivenson, N. T. Yardimci, M. Veli, Y. Luo, M. Jarrahi, and A. Ozcan (2018), Science 361 (6406), 1004.

Liu, D., S.-J. Ran, P. Wittek, C. Peng, R. B. García, G. Su, and M. Lewenstein (2017a), arXiv:1710.04833 [cond-mat, physics:physics, physics:quant-ph, stat] ArXiv: 1710.04833.

Liu, J., Y. Qi, Z. Y. Meng, and L. Fu (2017b), Physical Review B 95 (4), 041101.

Liu, J., H. Shen, Y. Qi, Z. Y. Meng, and L. Fu (2017c), Physical Review B 95 (24), 241104

Liu, K., J. Greitemann, L. Pollet, et al. (2019), Physical Review B 99 (10), 104410.

Liu, Y., X. Zhang, M. Lewenstein, and S.-J. Ran (2018), arXiv:1803.09111 [cond-mat, physics:quant-ph, stat] ArXiv: 1803.09111.

Lloyd, S., M. Mohseni, and P. Rebentrost (2014), Nature Physics 10, 631.

Louppe, G., K. Cho, C. Becot, and K. Cranmer (2017a), arXiv:1702.00748 [hep-ph]

Louppe, G., J. Hermans, and K. Cranmer (2017b), arXiv:1707.07113 [stat.ML]

Louppe, G., M. Kagan, and K. Cranmer (2016), arXiv:1611.01046 [stat.ME]

Lu, S., X. Gao, and L.-M. Duan (2018), arXiv:1810.02352 [cond-mat, physics:quant-ph] ArXiv: 1810.02352.

Lu, T., S. Wu, X. Xu, and T. Francis (1989), Applied optics $28(22), 4908$.

Lubbers, N., J. S. Smith, and K. Barros (2018), J. Chem. Phys. 148 (24), 241715.

Lundberg, K. H. (2005), IEEE Control Systems 25 (3), 22.

Luo, D., and B. K. Clark (2018), arXiv:1807.10770 [condmat, physics:physics] ArXiv: 1807.10770.

Mannelli, S. S., G. Biroli, C. Cammarota, F. Krzakala, P. Urbani, and L. Zdeborová (2018), arXiv preprint arXiv:1812.09066.

Mannelli, S. S., F. Krzakala, P. Urbani, and L. Zdeborová (2019), arXiv preprint arXiv:1902.00139.

Mardt, A., L. Pasquali, H. Wu, and F. Noé (2018), Nat. Commun. 9, 5 .

Marin, J.-M., P. Pudlo, C. P. Robert, and R. J. Ryder (2012), Statistics and Computing, 1.

Marjoram, P., J. Molitor, V. Plagnol, and S. Tavaré (2003), Proceedings of the National Academy of Sciences 100 (26), 15324.

Markidis, S., S. W. Der Chien, E. Laure, I. B. Peng, and J. S. Vetter (2018), IEEE International Parallel and Distributed Processing Symposium Workshops (IPDPSW). 
Marshall, P. J., D. W. Hogg, L. A. Moustakas, C. D. Fassnacht, M. Bradač, T. Schrabback, and R. D. Blandford (2009), The Astrophysical Journal 694 (2), 924.

Martiniani, S., P. M. Chaikin, and D. Levine (2019), Phys. Rev. X 9, 011031.

Maskara, N., A. Kubica, and T. Jochym-O'Connor (2019), Physical Review A 99 (5), 052351.

Matsushita, R., and T. Tanaka (2013), in Advances in Neural Information Processing Systems, pp. 917-925.

Mavadia, S., V. Frey, J. Sastrawan, S. Dona, and M. J. Biercuk (2017), Nature Communications 8, 14106.

McClean, J. R., J. Romero, R. Babbush, and A. AspuruGuzik (2016), New Journal of Physics 18 (2), 023023.

Mehta, P., M. Bukov, C.-H. Wang, A. G. Day, C. Richardson, C. K. Fisher, and D. J. Schwab (2018), arXiv preprint arXiv:1803.08823.

Mehta, P., and D. J. Schwab (2014), arXiv preprint arXiv:1410.3831.

Mei, S., A. Montanari, and P.-M. Nguyen (2018), arXiv preprint arXiv:1804.06561.

Melnikov, A. A., H. P. Nautrup, M. Krenn, V. Dunjko, M. Tiersch, A. Zeilinger, and H. J. Briegel (2018), Proceedings of the National Academy of Sciences 115 (6), 1221.

Metodiev, E. M., B. Nachman, and J. Thaler (2017), JHEP 10, 174 arXiv:1708.02949 [hep-ph]

Mézard, M. (2017), Physical Review E 95 (2), 022117.

Mézard, M., and A. Montanari (2009), Information, physics, and computation (Oxford University Press).

Mezzacapo, F., N. Schuch, M. Boninsegni, and J. I. Cirac (2009), New Journal of Physics 11 (8), 083026

Mills, K., K. Ryczko, I. Luchak, A. Domurad, C. Beeler, and I. Tamblyn (2019), Chem. Sci. 10 (15), 4129.

Minsky, M., and S. Papert (1969), Perceptrons: An Introduction to Computational Geometry (MIT Press, Cambridge, MA, USA).

Mitarai, K., M. Negoro, M. Kitagawa, and K. Fujii (2018), arXiv preprint arXiv:1803.00745.

Morningstar, A., and R. G. Melko (2018), Journal of Machine Learning Research 18 (163), 1.

Morningstar, W. R., Y. D. Hezaveh, L. Perreault Levasseur, R. D. Blandford, P. J. Marshall, P. Putzky, and R. H. Wechsler (2018), arXiv e-prints , arXiv:1808.00011 arXiv:1808.00011 [astro-ph.IM].

Morningstar, W. R., L. Perreault Levasseur, Y. D. Hezaveh, R. Blandford, P. Marshall, P. Putzky, T. D. Rueter, R. Wechsler, and M. Welling (2019), arXiv e-prints , arXiv:1901.01359 arXiv:1901.01359 [astro-ph.IM],

Nagai, R., R. Akashi, S. Sasaki, and S. Tsuneyuki (2018), J. Chem. Phys. 148 (24), 241737.

Nagai, Y., H. Shen, Y. Qi, J. Liu, and L. Fu (2017), Physical Review B 96 (16), 161102

Nagy, A., and V. Savona (2019), arXiv:1902.09483 [cond-mat, physics:quant-ph]

Nautrup, H. P., N. Delfosse, V. Dunjko, H. J. Briegel, and N. Friis (2018), arXiv preprint arXiv:1812.08451.

Ng, A. Y., M. I. Jordan, and Y. Weiss (2002), in Advances in neural information processing systems, pp. 849-856.

Nguyen, H. C., R. Zecchina, and J. Berg (2017), Advances in Physics 66 (3), 197.

Nguyen, T. T., E. Székely, G. Imbalzano, J. Behler, G. Csányi, M. Ceriotti, A. W. Götz, and F. Paesani (2018), J. Chem. Phys. 148 (24), 241725.

Nielsen, M. A., and I. Chuang (2002), "Quantum computation and quantum information,". van Nieuwenburg, E., E. Bairey, and G. Refael (2018), Physical Review B 98 (6), 060301

Nishimori, H. (2001), Statistical physics of spin glasses and information processing: an introduction, Vol. 111 (Clarendon Press).

Niu, M. Y., S. Boixo, V. Smelyanskiy, and H. Neven (2018), arXiv preprint arXiv:1803.01857.

Noé, F., S. Olsson, J. Köhler, and H. Wu (2019), Science 365 (6457).

Nomura, Y., A. S. Darmawan, Y. Yamaji, and M. Imada (2017), Physical Review B 96 (20), 205152.

Novikov, A., M. Trofimov, and I. Oseledets (2016), arXiv:1605.03795 ArXiv: 1605.03795.

Ntampaka, M., H. Trac, D. J. Sutherland, N. Battaglia, B. Póczos, and J. Schneider (2015), Astrophys. J. 803, 50 arXiv:1410.0686 [astro-ph.CO].

Ntampaka, M., H. Trac, D. J. Sutherland, S. Fromenteau, B. Póczos, and J. Schneider (2016), Astrophys. J. 831, 135 arXiv:1509.05409 [astro-ph.CO]

Ntampaka, M., J. ZuHone, D. Eisenstein, D. Nagai, A. Vikhlinin, L. Hernquist, F. Marinacci, D. Nelson, R. Pakmor, A. Pillepich, P. Torrey, and M. Vogelsberger (2018), arXiv e-prints , arXiv:1810.07703arXiv:1810.07703 [astro-ph.CO].

Ntampaka, M., et al. (2019), arXiv:1902.10159 [astro-ph.IM]

Nussinov, Z., P. Ronhovde, D. Hu, S. Chakrabarty, B. Sun, N. A. Mauro, and K. K. Sahu (2016), in Information Science for Materials Discovery and Design (Springer) pp. $115-138$

O'Donnell, R., and J. Wright (2016), in Proceedings of the forty-eighth annual ACM symposium on Theory of Computing (ACM) pp. 899-912.

Ohtsuki, T., and T. Ohtsuki (2016), Journal of the Physical Society of Japan 85 (12), 123706

Ohtsuki, T., and T. Ohtsuki (2017), Journal of the Physical Society of Japan 86 (4), 044708

de Oliveira, L., M. Kagan, L. Mackey, B. Nachman, and A. Schwartzman (2016), JHEP 07, 069, arXiv:1511.05190 [hep-ph].

Oseledets, I. (2011), SIAM Journal on Scientific Computing 33 (5), 2295.

Paganini, M., L. de Oliveira, and B. Nachman (2018a), Phys. Rev. Lett. 120 (4), 042003 arXiv:1705.02355 [hep-ex]

Paganini, M., L. de Oliveira, and B. Nachman (2018b), Phys. Rev. D97 (1), 014021, arXiv:1712.10321 [hep-ex]

Pang, L.-G., K. Zhou, N. Su, H. Petersen, H. Stöcker, and X.-N. Wang (2018), Nature Commun. 9 (1), 210 , arXiv:1612.04262 [hep-ph]

Papamakarios, G., I. Murray, and T. Pavlakou (2017), in Advances in Neural Information Processing Systems, pp. 2335-2344

Papamakarios, G., D. C. Sterratt, and I. Murray (2018), arXiv e-prints , arXiv:1805.07226arXiv:1805.07226 [stat.ML],

Paris, M., and J. Rehacek, Eds. (2004), Quantum State Estimation, Lecture Notes in Physics (Springer-Verlag, Berlin Heidelberg)

Paruzzo, F. M., A. Hofstetter, F. Musil, S. De, M. Ceriotti, and L. Emsley (2018), Nat. Commun. 9, 4501.

Pastori, L., R. Kaubruegger, and J. C. Budich (2018), arXiv:1808.02069 [cond-mat, physics:physics, physics:quant-ph] ArXiv: 1808.02069.

Pathak, J., B. Hunt, M. Girvan, Z. Lu, and E. Ott (2018), Physical review letters 120 (2), 024102. 
Pathak, J., Z. Lu, B. R. Hunt, M. Girvan, and E. Ott (2017), Chaos: An Interdisciplinary Journal of Nonlinear Science 27 (12), 121102.

Peel, A., F. Lalande, J.-L. Starck, V. Pettorino, J. Merten, C. Giocoli, M. Meneghetti, and M. Baldi (2018), arXiv eprints , arXiv:1810.11030 arXiv:1810.11030 [astro-ph.CO]

Perdomo-Ortiz, A., M. Benedetti, J. Realpe-Gómez, and R. Biswas (2017), arXiv preprint arXiv:1708.09757.

Póczos, B., L. Xiong, D. J. Sutherland, and J. G. Schneider (2012), CoRR abs/1202.0302, arXiv:1202.0302

Putzky, P., and M. Welling (2017), arXiv preprint arXiv:1706.04008.

Quek, Y., S. Fort, and H. K. Ng (2018), arXiv:1812.06693 [quant-ph] ArXiv: 1812.06693.

Radovic, A., M. Williams, D. Rousseau, M. Kagan, D. Bonacorsi, A. Himmel, A. Aurisano, K. Terao, and T. Wongji$\operatorname{rad}(2018)$, Nature 560 (7716), 41

Ramakrishnan, R., P. O. Dral, M. Rupp, and O. A. von Lilienfeld (2014), Sci. Data 1, 191.

Rangan, S., and A. K. Fletcher (2012), in Information Theory Proceedings (ISIT), 2012 IEEE International Symposium on (IEEE) pp. 1246-1250.

Ravanbakhsh, S., F. Lanusse, R. Mandelbaum, J. Schneider, and B. Poczos (2016), arXiv e-prints , arXiv:1609.05796 arXiv:1609.05796 [astro-ph.IM],

Ravanbakhsh, S., J. Oliva, S. Fromenteau, L. C. Price, S. Ho, J. Schneider, and B. Poczos (2017), arXiv e-prints , arXiv:1711.02033 arXiv:1711.02033 [astro-ph.CO].

Reck, M., A. Zeilinger, H. J. Bernstein, and P. Bertani (1994), Physical Review Letters $\mathbf{7 3}$ (1), 58.

Reddy, G., A. Celani, T. J. Sejnowski, and M. Vergassola (2016), Proceedings of the National Academy of Sciences 113 (33), E4877.

Reddy, G., J. Wong-Ng, A. Celani, T. J. Sejnowski, and M. Vergassola (2018), Nature 562 (7726), 236.

Regier, J., A. C. Miller, D. Schlegel, R. P. Adams, J. D. McAuliffe, and Prabhat (2018), arXiv e-prints, arXiv:1803.00113 arXiv:1803.00113 [stat.AP]

Rem, B. S., N. Käming, M. Tarnowski, L. Asteria, N. Fläschner, C. Becker, K. Sengstock, and C. Weitenberg (2018), arXiv:1809.05519 [cond-mat, physics:quantph] ArXiv: 1809.05519.

Ren, S., K. He, R. Girshick, and J. Sun (2015), in Advances in neural information processing systems, pp. 91-99.

Rezende, D., and S. Mohamed (2015), in International Conference on Machine Learning, pp. 1530-1538, 1505.05770

Rezende, D. J., S. Mohamed, and D. Wierstra (2014), in Proceedings of the 31st International Conference on International Conference on Machine Learning-Volume 32 (JMLR. org) pp. II-1278.

Riofrío, C. A., D. Gross, S. T. Flammia, T. Monz, D. Nigg, R. Blatt, and J. Eisert (2017), Nature Communications 8, 15305

Ritzmann, U., S. von Malottki, J.-V. Kim, S. Heinze, J. Sinova, and B. Dupé (2018), Nature Electronics 1 (8), 451 .

Robin, A. C., C. Reylé, J. Fliri, M. Czekaj, C. P. Robert, and A. M. M. Martins (2014), Astronomy and Astrophysics 569, A13, arXiv:1406.5384 [astro-ph.GA]

Rocchetto, A. (2018), Quantum Information and Computation 18 (7\&8).

Rocchetto, A., S. Aaronson, S. Severini, G. Carvacho, D. Poderini, I. Agresti, M. Bentivegna, and F. Sciarrino (2017), arXiv:1712.00127 [quant-ph] ArXiv: 1712.00127.
Rocchetto, A., E. Grant, S. Strelchuk, G. Carleo, and S. Severini (2018), npj Quantum Information 4 (1), 28.

Rodríguez, A. C., T. Kacprzak, A. Lucchi, A. Amara, R. Sgier, J. Fluri, T. Hofmann, and A. Réfrégier (2018), Computational Astrophysics and Cosmology 5, 4 , arXiv:1801.09070 [astro-ph.CO]

Rodriguez-Nieva, J. F., and M. S. Scheurer (2018), arXiv:1805.05961 [cond-mat] ArXiv: 1805.05961.

Roe, B. P., H.-J. Yang, J. Zhu, Y. Liu, I. Stancu, and G. McGregor (2005), Nucl. Instrum. Meth. A543 (2-3), 577, arXiv:physics/0408124 [physics].

Rogozhnikov, A., A. Bukva, V. V. Gligorov, A. Ustyuzhanin, and M. Williams (2015), JINST 10 (03), T03002, arXiv:1410.4140 [hep-ex]

Ronhovde, P., S. Chakrabarty, D. Hu, M. Sahu, K. Sahu, K. Kelton, N. Mauro, and Z. Nussinov (2011), The European Physical Journal E 34 (9), 105.

Rotskoff, G., and E. Vanden-Eijnden (2018), in Advances in Neural Information Processing Systems, pp. 7146-7155.

Rupp, M., O. A. von Lilienfeld, and K. Burke (2018), J. Chem. Phys. 148 (24), 241401.

Rupp, M., A. Tkatchenko, K.-R. Müller, and O. A. von Lilienfeld (2012), Phys. Rev. Lett. 108, 058301.

Saad, D., and S. A. Solla (1995a), Physical Review Letters 74 (21), 4337.

Saad, D., and S. A. Solla (1995b), Physical Review E 52 (4), 4225.

Saade, A., F. Caltagirone, I. Carron, L. Daudet, A. Drémeau, S. Gigan, and F. Krzakala (2016), in Acoustics, Speech and Signal Processing (ICASSP), 2016 IEEE International Conference on (IEEE) pp. 6215-6219.

Saade, A., F. Krzakala, and L. Zdeborová (2014), in Advances in Neural Information Processing Systems, pp. 406-414.

Saito, H. (2017), Journal of the Physical Society of Japan 86 (9), 093001

Saito, H. (2018), Journal of the Physical Society of Japan 87 (7), 074002.

Saito, H., and M. Kato (2017), Journal of the Physical Society of Japan 87 (1), 014001.

Sakata, A., and Y. Kabashima (2013), EPL (Europhysics Letters) 103 (2), 28008.

Saxe, A. M., Y. Bansal, J. Dapello, M. Advani, A. Kolchinsky, B. D. Tracey, and D. D. Cox (2018),

Saxe, A. M., J. L. McClelland, and S. Ganguli (2013), in ICLR 2014, arXiv preprint arXiv:1312.6120.

Schawinski, K., C. Zhang, H. Zhang, L. Fowler, and G. K. Santhanam (2017), Monthly Notices of the Royal Astronomical Society: Letters, slx008ArXiv: 1702.00403.

Schindler, F., N. Regnault, and T. Neupert (2017), Physical Review B 95 (24), 245134.

Schmidhuber, J. (2014), CoRR abs/1404.7828 arXiv:1404.7828

Schmidt, E., A. T. Fowler, J. A. Elliott, and P. D. Bristowe (2018), Comput. Mater. Sci. 149, 250.

Schmitt, M., and M. Heyl (2018), SciPost Physics 4 (2), 013.

Schneider, E., L. Dai, R. Q. Topper, C. Drechsel-Grau, and M. E. Tuckerman (2017), Phys. Rev. Lett. 119 (15), 150601.

Schoenholz, S. S., E. D. Cubuk, E. Kaxiras, and A. J. Liu (2017), Proceedings of the National Academy of Sciences 114 (2), 263.

Schuch, N., M. M. Wolf, F. Verstraete, and J. I. Cirac (2008), Physical Review Letters 100 (4), 040501. 
Schuld, M., and N. Killoran (2018), arXiv preprint arXiv:1803.07128v1.

Schuld, M., and F. Petruccione (2018a), Quantum computing for supervised learning (Springer).

Schuld, M., and F. Petruccione (2018b), Supervised Learning with Quantum Computers (Springer).

Schütt, K. T., H. E. Sauceda, P. J. Kindermans, A. Tkatchenko, and K. R. Müller (2018), J. Chem. Phys. 148 (24), 241722.

Schwarze, H. (1993), Journal of Physics A: Mathematical and General 26 (21), 5781.

Seif, A., K. A. Landsman, N. M. Linke, C. Figgatt, C. Monroe, and M. Hafezi (2018), Journal of Physics B: Atomic, Molecular and Optical Physics 51 (17), 174006 arXiv: 1804.07718.

Seung, H., H. Sompolinsky, and N. Tishby (1992a), Physical Review A 45 (8), 6056.

Seung, H. S., M. Opper, and H. Sompolinsky (1992b), in Proceedings of the fifth annual workshop on Computational learning theory (ACM) pp. 287-294.

Shanahan, P. E., D. Trewartha, and W. Detmold (2018), Phys. Rev. D97 (9), 094506, arXiv:1801.05784 [hep-lat].

Sharir, O., Y. Levine, N. Wies, G. Carleo, and A. Shashua (2019), arXiv:1902.04057 [cond-mat]

Shen, H., D. George, E. A. Huerta, and Z. Zhao (2019), arXiv:1903.03105 [astro-ph.CO]

Shen, Y., N. C. Harris, S. Skirlo, M. Prabhu, T. Baehr-Jones, M. Hochberg, X. Sun, S. Zhao, H. Larochelle, D. Englund, et al. (2017), Nature Photonics 11 (7), 441.

Shi, Y.-Y., L.-M. Duan, and G. Vidal (2006), Physical Review A 74 (2), 022320.

Shimmin, C., P. Sadowski, P. Baldi, E. Weik, D. Whiteson, E. Goul, and A. Søgaard (2017), Phys. Rev. D96 (7), 074034, arXiv:1703.03507 [hep-ex]

Shwartz-Ziv, R., and N. Tishby (2017), arXiv preprint arXiv:1703.00810.

Sidky, H., and J. K. Whitmer (2018), J. Chem. Phys. 148 (10), 104111.

Sifain, A. E., N. Lubbers, B. T. Nebgen, J. S. Smith, A. Y. Lokhov, O. Isayev, A. E. Roitberg, K. Barros, and S. Tretiak (2018), J. Phys. Chem. Lett. 9 (16), 4495.

Sisson, S. A., and Y. Fan (2011), Likelihood-free MCMC (Chapman \& Hall/CRC, New York.[839]).

Sisson, S. A., Y. Fan, and M. M. Tanaka (2007), Proceedings of the National Academy of Sciences 104 (6), 1760.

Smith, J. S., O. Isayev, and A. E. Roitberg (2017), Chem. Sci. 8 (4), 3192.

Smith, J. S., B. Nebgen, N. Lubbers, O. Isayev, and A. E. Roitberg (2018), J. Chem. Phys. 148 (24), 241733.

Smolensky, P. (1986), Chap. Information Processing in Dynamical Systems: Foundations of Harmony Theory (MIT Press, Cambridge, MA, USA) pp. 194-281.

Snyder, J. C., M. Rupp, K. Hansen, K.-R. Müller, and K. Burke (2012), Phys. Rev. Lett. 108 (25), 1875.

Sompolinsky, H., N. Tishby, and H. S. Seung (1990), Physical Review Letters 65 (13), 1683.

Sorella, S. (1998), Physical Review Letters 80 (20), 4558

Sosso, G. C., V. L. Deringer, S. R. Elliott, and G. Csányi (2018), Mol. Simulat. 44 (11), 866.

Steinbrecher, G. R., J. P. Olson, D. Englund, and J. Carolan (2018), "Quantum optical neural networks," arxiv:1808.10047

Stevens, J., and M. Williams (2013), JINST 8, P12013, arXiv:1305.7248 [nucl-ex].
Stokes, J., and J. Terilla (2019), arXiv preprint arXiv:1902.06888.

Stoudenmire, E., and D. J. Schwab (2016), in Advances in Neural Information Processing Systems 29, edited by D. D. Lee, M. Sugiyama, U. V. Luxburg, I. Guyon, and R. Garnett (Curran Associates, Inc.) pp. 4799-4807.

Stoudenmire, E. M. (2018), Quantum Science and Technology $3(3), 034003$

Sun, N., J. Yi, P. Zhang, H. Shen, and H. Zhai (2018), Physical Review B 98 (8), 085402

Sutton, R. S., and A. G. Barto (2018), Reinforcement learning: An introduction (MIT press).

Sweke, R., M. S. Kesselring, E. P. van Nieuwenburg, and J. Eisert (2018), arXiv preprint arXiv:1810.07207.

Tanaka, A., and A. Tomiya (2017a), Journal of the Physical Society of Japan 86 (6), 063001

Tanaka, A., and A. Tomiya (2017b), arXiv:1712.03893 [heplat]

Tang, E. (2018), arXiv preprint arXiv:1807.04271.

Teng, P. (2018), Physical Review E 98 (3), 033305

Thouless, D. J., P. W. Anderson, and R. G. Palmer (1977), Philosophical Magazine 35 (3), 593.

Tiersch, M., E. Ganahl, and H. J. Briegel (2015), Scientific reports 5, 12874 .

Tishby, N., F. C. Pereira, and W. Bialek (2000), arXiv preprint physics $/ 0004057$.

Tishby, N., and N. Zaslavsky (2015), in Information Theory Workshop (ITW), 2015 IEEE (IEEE) pp. 1-5.

Torlai, G., G. Mazzola, J. Carrasquilla, M. Troyer, R. Melko, and G. Carleo (2018), Nature Physics 14 (5), 447.

Torlai, G., and R. G. Melko (2017), Physical Review Letters 119 (3), 030501

Torlai, G., and R. G. Melko (2018), Physical Review Letters 120 (24), 240503

Torlai, G., B. Timar, E. P. L. van Nieuwenburg, H. Levine, A. Omran, A. Keesling, H. Bernien, M. Greiner, V. Vuletić, M. D. Lukin, R. G. Melko, and M. Endres (2019), arXiv:1904.08441 [cond-mat, physics:quant-ph] ArXiv: 1904.08441.

Tóth, G., W. Wieczorek, D. Gross, R. Krischek, C. Schwemmer, and H. Weinfurter (2010), Physical review letters 105 (25), 250403.

Tramel, E. W., M. Gabrié, A. Manoel, F. Caltagirone, and F. Krzakala (2018), Physical Review X 8 (4), 041006.

Tsaris, A., et al. (2018), Proceedings, 18th International Workshop on Advanced Computing and Analysis Techniques in Physics Research (ACAT 2017): Seattle, WA, USA, August 21-25, 2017, J. Phys. Conf. Ser. 1085 (4), 042023.

Tubiana, J., S. Cocco, and R. Monasson (2018), arXiv preprint arXiv:1803.08718.

Tubiana, J., and R. Monasson (2017), Physical review letters 118 (13), 138301.

Uria, B., M.-A. Côté, K. Gregor, I. Murray, and H. Larochelle (2016), Journal of Machine Learning Research 17 (205), 1.

Valiant, L. G. (1984), Communications of the ACM 27 (11), 1134.

Van Nieuwenburg, E. P., Y.-H. Liu, and S. D. Huber (2017), Nature Physics 13 (5), 435.

Varsamopoulos, S., K. Bertels, and C. G. Almudever (2018), arXiv preprint arXiv:1811.12456.

Varsamopoulos, S., K. Bertels, and C. G. Almudever (2019), arXiv preprint arXiv:1901.10847.

Varsamopoulos, S., B. Criger, and K. Bertels (2017), Quantum Science and Technology 3 (1), 015004. 
Venderley, J., V. Khemani, and E.-A. Kim (2018), Physical Review Letters 120 (25), 257204.

Verstraete, F., V. Murg, and J. I. Cirac (2008), Advances in Physics 57 (2), 143

Vicentini, F., A. Biella, N. Regnault, and C. Ciuti (2019), arXiv:1902.10104 [cond-mat, physics:quant-ph]

ArXiv: 1902.10104.

Vidal, G. (2007), Physical Review Letters 99 (22), 220405.

Von Luxburg, U. (2007), Statistics and computing 17 (4), 395.

Wang, C., H. Hu, and Y. M. Lu (2018), arXiv preprint arxXiv:1805.08349.

Wang, C., and H. Zhai (2017), Physical Review B 96 (14), 144432.

Wang, C., and H. Zhai (2018), Frontiers of Physics 13 (5), 130507.

Wang, L. (2016), Physical Review B 94 (19), 195105

Wang, L. (2018), "Generative models for physicists,".

Watkin, T., and J.-P. Nadal (1994), Journal of Physics A: Mathematical and General 27 (6), 1899.

Wecker, D., M. B. Hastings, and M. Troyer (2016), Physical Review A 94 (2), 022309.

Wehmeyer, C., and F. Noé (2018), J. Chem. Phys. 148 (24), 241703.

Wetzel, S. J. (2017), Physical Review E 96 (2), 022140.

White, S. R. (1992), Physical Review Letters 69 (19), 2863

Wigley, P. B., P. J. Everitt, A. van den Hengel, J. W. Bastian, M. A. Sooriyabandara, G. D. McDonald, K. S. Hardman, C. D. Quinlivan, P. Manju, C. C. N. Kuhn, I. R. Petersen, A. N. Luiten, J. J. Hope, N. P. Robins, and M. R. Hush (2016), Scientific Reports 6, 25890.

Wu, D., L. Wang, and P. Zhang (2018), arXiv preprint arXiv:1809.10606.

Xin, T., S. Lu, N. Cao, G. Anikeeva, D. Lu, J. Li, G. Long, and B. Zeng (2018), arXiv:1807.07445 [quant-ph] ArXiv: 1807.07445 .
Xu, Q., and S. Xu (2018), arXiv:1811.06654 [quant-ph] ArXiv: 1811.06654.

Yao, K., J. E. Herr, D. W. Toth, R. Mckintyre, and J. Parkhill (2018), Chem. Sci. 9 (8), 2261.

Yedidia, J. S., W. T. Freeman, and Y. Weiss (2003), Exploring artificial intelligence in the new millennium 8, 236.

Yoon, H., J.-H. Sim, and M. J. Han (2018), Physical Review B $98(24), 245101$

Yoshioka, N., and R. Hamazaki (2019), arXiv:1902.07006 [cond-mat, physics:quant-ph].

Zdeborová, L., and F. Krzakala (2016), Advances in Physics 65 (5), 453.

Zhang, C., S. Bengio, M. Hardt, B. Recht, and O. Vinyals (2016), arXiv preprint arXiv:1611.03530.

Zhang, L., J. Han, H. Wang, R. Car, and W. E (2018a), Phys. Rev. Lett. 120 (14), 143001.

Zhang, L., D.-Y. Lin, H. Wang, R. Car, et al. (2018b), arXiv preprint arXiv:1810.11890.

Zhang, P., H. Shen, and H. Zhai (2018c), Physical Review Letters 120 (6), 066401

Zhang, W., L. Wang, and Z. Wang (2019), Physical Review B 99 (5), 054208.

Zhang, X., Y. Wang, W. Zhang, Y. Sun, S. He, G. Contardo, F. Villaescusa-Navarro, and S. Ho (2019), arXiv e-prints, arXiv:1902.05965arXiv:1902.05965 [astro-ph.CO]

Zhang, X.-M., Z. Wei, R. Asad, X.-C. Yang, and X. Wang (2019), arXiv preprint arXiv:1902.02157.

Zhang, Y., and E.-A. Kim (2017), Physical Review Letters 118 (21), 216401

Zhang, Y., R. G. Melko, and E.-A. Kim (2017), Physical Review B 96 (24), 245119.

Zhang, Y., A. Mesaros, K. Fujita, S. D. Edkins, M. H. Hamidian, K. Ch'ng, H. Eisaki, S. Uchida, J. C. S. Davis, E. Khatami, and E.-A. Kim (2018d), arXiv:1808.00479 [cond-mat, physics:physics].

Zheng, Y., H. He, N. Regnault, and B. A. Bernevig (2018), arXiv:1812.08171 